\title{
Indirect probes of the MSSM after the Higgs discovery
}

\author{
Wolfgang Altmannshofer, ${ }^{a}$ Marcela Carena, ${ }^{a, b, c}$ Nausheen R. Shah ${ }^{d}$ and Felix $\mathbf{Y u}^{a}$ \\ ${ }^{a}$ Fermi National Accelerator Laboratory, \\ P.O. Box 500, Batavia, IL 60510, U.S.A. \\ ${ }^{b}$ Enrico Fermi Institute, University of Chicago, \\ Chicago, IL 60637, U.S.A. \\ ${ }^{c}$ Kavli Institute for Cosmological Physics, University of Chicago, \\ Chicago, IL 6063\%, U.S.A. \\ ${ }^{d}$ Michigan Center for Theoretical Physics, Department of Physics, University of Michigan, \\ Ann Arbor, MI 48109, U.S.A. \\ E-mail: waltmann@fnal.gov, carena@fnal.gov, naushah@umich.edu, \\ felixyu@fnal.gov
}

ABSTRACT: We study the minimal supersymmetric standard model (MSSM) with minimal flavor violation (MFV), imposing constraints from flavor physics observables and MSSM Higgs searches, in light of the recent discovery of a $125 \mathrm{GeV}$ Higgs boson by ATLAS and CMS. We analyze the electroweak vacuum stability conditions to further restrict the MSSM parameter space. In addition, a connection to ultraviolet physics is shown via an implementation of renormalization group running, which determines the TeV-scale spectrum from a small set of minimal supergravity parameters. Finally, we investigate the impact from dark matter direct detection searches. Our work highlights the complementarity of collider, flavor and dark matter probes in exploring the MSSM, and shows that even in a MFV framework, flavor observables constrain the MSSM parameter space well beyond the current reach of direct SUSY particle searches.

KeYwords: Supersymmetry Phenomenology

ARXIV EPRINT: 1211.1976 


\section{Contents}

1 Introduction 1

2 The MSSM with minimal flavor violation 3

2.1 Sfermion spectrum 3

2.2 Higgs spectrum 5

2.3 Higgs couplings to fermions $\quad 6$

3 Higgs collider searches $\quad 9$

3.1 SM-like $125 \mathrm{GeV}$ Higgs 9

$\begin{array}{lll}3.2 & \text { Searches for heavy scalars and pseudoscalars } & 10\end{array}$

4 Vacuum stability 12

$\begin{array}{lll}5 & B \text { physics observables } & \mathbf{1 5}\end{array}$

$5.1 \quad B \rightarrow \tau \nu, B \rightarrow D^{(*)} \tau \nu$ and $K \rightarrow \mu \nu \quad 15$

$\begin{array}{lll}5.2 B_{s} \rightarrow \mu^{+} \mu^{-} & 19\end{array}$

$5.3 B \rightarrow X_{s} \gamma \quad 24$

5.4 Discussion of RGE effects 28

6 Dark matter direct detection 33

$\begin{array}{lll}7 & \text { Conclusions } & 36\end{array}$

$\begin{array}{ll}\text { A Loop functions } & 38\end{array}$

\section{Introduction}

Despite the null results for direct searches of supersymmetric particles at the LHC [1-29], models of supersymmetry (SUSY) remain among the most well-motivated and popular extensions of the Standard Model (SM). Besides direct searches, there exist numerous ways to indirectly probe SUSY models, e.g. with low energy flavor observables, from dark matter direct detection results, and through Higgs properties. The discovery of a new particle at the LHC with a mass of $\sim 125 \mathrm{GeV}$ compatible with a SM-like Higgs boson [30, 31] has far reaching consequences for any model of New Physics (NP) with a non-standard Higgs sector. Indeed, the LHC Higgs results have motivated numerous studies of their implications in the context of the Minimal Supersymmetric Standard Model (MSSM) and its variants [32-75].

A SM-like Higgs with a mass of $M_{h} \simeq 125 \mathrm{GeV}$ can be accommodated in the MSSM as long as stops are either very heavy or strongly mixed. Interestingly enough, large stop 
mixing unavoidably leads to irreducible contributions to low energy flavor observables, in particular in the Flavor Changing Neutral Current (FCNC) decays $B_{s} \rightarrow \mu^{+} \mu^{-}$and $B \rightarrow X_{s} \gamma$, even if all soft masses are flavor blind. Correspondingly, rare $B$ decays can be used to set non-trivial constraints on MSSM parameters.

In this work, we discuss the status of the MSSM, in view of the recent Higgs search results from the LHC $[30,31]$ and the Tevatron [76], the recent strong limits on MSSM Higgs bosons in $H / A \rightarrow b \bar{b}$ searches [78, 79] and $H / A \rightarrow \tau^{+} \tau^{-}$searches [77], the latest results in $B$ physics, in particular the recent evidence for $B_{s} \rightarrow \mu^{+} \mu^{-}$from LHCb [80], the updated results on $B \rightarrow \tau \nu$ from Belle [81] and BaBar [82] as well as on $B \rightarrow X_{s} \gamma$ from BaBar [83], and also the updated Xenon100 bounds on dark matter direct detection [84]. We will assume that the flavor structure of the SUSY particles is determined by the principle of minimal flavor violation (MFV) [85-88], i.e. we will assume that the SM Yukawa couplings are the only sources of flavor violation. This is motivated by the absence of any unambiguous deviation from SM expectations in flavor observables. We emphasize that even in this restrictive framework, flavor observables play an important role in constraining the viable parameter space of the MSSM. Indeed, flavor bounds can be stronger than bounds from direct searches for SUSY particles in various regions of parameter space. This is particularly true for large values of $\tan \beta$, where loop-induced flavor changing couplings of the heavy Higgs bosons of the MSSM give enhanced contributions to FCNC processes. In the MSSM with large $\tan \beta$, direct searches of the heavy Higgs bosons also become especially sensitive and, moreover, the exchange of heavy Higgs bosons can also lead to large dark matter direct detection cross sections, giving additional complementary means to probe this region of parameter space.

In the large $\tan \beta$ regime, loop corrections to Higgs-fermion couplings can be significant and it is mandatory to resum $\tan \beta$-enhanced terms to obtain reliable predictions for any observables that depend on these couplings in the MSSM. We provide comprehensive analytical expressions for all the relevant loop-corrected Higgs couplings, loop corrections to the SM-like Higgs mass, Higgs production and decay rates, contributions to flavor observables, and dark matter direct detection cross sections, consistently taking into account the most general structure of the soft SUSY breaking terms compatible with the MFV ansatz. In particular, we include effects from the bottom Yukawa coupling as well as the tau Yukawa coupling, as they are relevant for large $\tan \beta$. This goes beyond the analyses in [89-91], where bottom Yukawa effects in the squark masses were neglected.

We also give a detailed treatment of gaugino loop contributions to FCNC processes that can arise from a mass splitting between the left-handed squarks of the first two and the third generations. We highlight that in order to discuss the gaugino contributions to FCNC processes in the large $\tan \beta$ regime, both the squark mass splitting and the alignment of this splitting with the quark Yukawas must be considered.

Putting together all the relevant experimental constraints coming from current Higgs, flavor and dark matter sectors on the MSSM parameters, we point out regions of the MFV MSSM parameter space where these constraints are minimized. We also discuss the robustness of these bounds and to which extent they can be avoided. We take a phenomenological approach and treat the MSSM parameters as free parameters at the $\mathrm{TeV}$ 
scale: however, we augment this discussion with a study of renormalization group equation (RGE) effects, assuming minimal supergravity (mSUGRA)-like boundary conditions at a high scale and monitoring the generic spectrum of SUSY particles and their mass splittings induced by the running.

In section 2, we review the MSSM with minimal flavor violation in the quark sector. The impact of Higgs searches at the LHC and Tevatron on the MSSM are analyzed in section 3. We use the recent results indicating the presence of a SM-like Higgs as well as dedicated searches for the additional Higgs bosons of the MSSM. In section 4, we study constraints on large $\mu \tan \beta$ from vacuum stability considerations. Constraints from $B$ physics observables are analyzed in section 5 . In section 6 , bounds on the MSSM from dark matter direct detection are considered. We conclude in section 7 .

\section{The MSSM with minimal flavor violation}

In the following, we briefly review the MSSM with MFV. Throughout this work, in addition to $\mathrm{MFV}$, we also assume minimal $C P$ violation, i.e. the phase of the CKM matrix is the only source of $C P$ violation, while all the MSSM parameters are $C P$ conserving. We discuss the MFV structure of the sfermion masses in section 2.1. Aspects of the Higgs spectrum that are relevant for our work are briefly reviewed in section 2.2. In section 2.3, we detail the $\tan \beta$-enhanced loop corrections to the Higgs-fermion couplings, allowing for the most general squark spectrum compatible with our MFV and $C P$ conservation assumptions.

\subsection{Sfermion spectrum}

The soft SUSY breaking terms that give mass to the squarks, i.e. the soft masses, $m_{Q}^{2}$, $m_{D}^{2}$ and $m_{U}^{2}$, and trilinear couplings, $A_{d}$ and $A_{u}$, are possible sources of flavor violation. Generic flavor violating entries in these matrices are strongly constrained by flavor physics data. A simple approach to address this "SUSY flavor problem" is the principle of minimal flavor violation [85-88], which states that the SM Yukawa couplings are the only sources of flavor violation even in extensions of the SM. In the context of the MSSM, this implies that the soft terms can be expanded in powers of the Yukawa couplings. In the super-CabibboKobayashi-Maskawa (super-CKM) basis, where squarks and quarks are simultaneously rotated to obtain diagonal Yukawa couplings, the soft masses are [85]

$$
\begin{aligned}
& \hat{m}_{Q}^{2}=\tilde{m}_{Q}^{2}\left(1+b_{1} V^{\dagger} y_{u}^{2} V+b_{2} y_{d}^{2}+b_{3}\left(y_{d}^{2} V^{\dagger} y_{u}^{2} V+V^{\dagger} y_{u}^{2} V y_{d}^{2}\right)\right), \\
& \hat{m}_{U}^{2}=\tilde{m}_{U}^{2}\left(1+b_{4} y_{u}^{2}\right) \\
& \hat{m}_{D}^{2}=\tilde{m}_{D}^{2}\left(1+b_{5} y_{d}^{2}\right)
\end{aligned}
$$

where $y_{u}$ and $y_{d}$ are the diagonal up and down quark Yukawa matrices and $V$ is the CKM matrix. The soft mass $\hat{m}_{Q}^{2}$ enters the left-left block of the down squark mass matrix, while $V \hat{m}_{Q}^{2} V^{\dagger}$ enters the up squark mass matrix. The generic structure in (2.1) is always generated by RGE running if flavor blind boundary conditions are assumed at a high scale $[92,93]$. The parameters $b_{i}$ lead to splittings between the squark masses. To be specific, the parameters $b_{4}$ and $b_{5}$ generate a splitting between the masses of the first 
two and the third generations of right-handed up and down squarks, respectively, while the parameters $b_{1}, b_{2}$, and $b_{3}$ generate a splitting between the first two and the third generations of left-handed squarks. Note that the parameters $b_{2}, b_{3}$, and $b_{5}$ only become important for large values of $\tan \beta$, where the bottom Yukawa is $\mathcal{O}(1)$. As we are particularly interested in the large $\tan \beta$ regime, in the following we will take all the above masses as independent parameters and use $m_{Q_{3}}^{2}, m_{D_{3}}^{2}$, and $m_{U_{3}}^{2}$ for the stop and sbottom masses and $m_{Q}^{2}, m_{D}^{2}$, and $m_{U}^{2}$ for the masses of the first two generations, which are degenerate to an excellent approximation in this setup. This is analogous to the pMSSM framework [94] frequently studied in the literature.

We stress, however, that the parameters $b_{1}, b_{2}$, and $b_{3}$ also induce flavor violating entries in the left-handed squark mass matrices. These entries are proportional to small CKM angles and lead to controlled but non-negligible contributions to FCNC processes. In fact, due to $\mathrm{SU}(2)_{L}$ invariance, the left-left blocks of the up and down squark mass matrices are related by a CKM rotation, and therefore any splitting in the diagonal entries of the lefthanded soft masses $\hat{m}_{Q}^{2}$ unavoidably induces off-diagonal entries in the up or down squark mass matrices. Moreover, distinct flavor phenomenology arises depending on which of the $b_{1}, b_{2}$, or $b_{3}$ parameters is responsible for the splitting. In particular, a splitting induced by $b_{1}\left(b_{2}\right)$ is aligned in the up- (down-) sector and will only lead to off-diagonal entries in the down (up) squark mass matrix. The parameter $b_{3}$ induces flavor violation in both the up and down squark masses matrices. All flavor observables that we will discuss in the following depend on the combination $b_{1}+b_{3} y_{b}^{2}$. We thus introduce one additional parameter

$$
\zeta=\frac{b_{1} y_{t}^{2}+b_{3} y_{b}^{2} y_{t}^{2}}{b_{1} y_{t}^{2}+b_{2} y_{b}^{2}+2 b_{3} y_{b}^{2} y_{t}^{2}},
$$

which reflects the alignment of the splitting in the left-handed squark masses and hence parametrizes the fraction of the splitting in the masses leading to flavor violation in the down sector. We assume $\zeta$ is real in the following. ${ }^{1}$ We see that formally $\zeta=1+\mathcal{O}\left(y_{b}^{2}\right)$. If we consider a splitting in the squark masses that is radiatively induced through RGE running, then considering only the top Yukawa in the running leads to $\zeta=1$. Bottom Yukawa effects become important for large $\tan \beta$ and can lead to $0<\zeta<1$. Typically we expect that $y_{b}$ is at most as large as $y_{t}$, however, which implies $1 / 2<\zeta<1$.

We note that an expansion analogous to (2.1) also exists for the trilinear couplings [85]. In particular, higher order terms in the expansion can lead to flavor violating trilinear terms. Such terms only lead to corrections of the holomorphic Higgs couplings, however. These corrections can induce flavor changing neutral Higgs couplings, that are especially interesting beyond MFV, where the corresponding effects can be chirally enhanced [95, 96]. In the MFV framework considered here, these effects are less important compared to contributions that are related to the loop-induced non-holomorphic Higgs couplings. The only relevant trilinear couplings for our analysis are those for the third generation squarks, $A_{t}$ and $A_{b}$, which we will take to be independent parameters.

\footnotetext{
${ }^{1}$ Note that while $b_{1}$ and $b_{2}$ have to be real due to hermiticity of the squark masses, $b_{3}$ can in principle be complex. Indeed, as shown in [93], a tiny phase for $b_{3}$ is always generated during RGE running.
} 
For simplicity, we will also assume universal soft masses $m_{L}^{2}$ and $m_{E}^{2}$, in the slepton sector. The phenomenology of flavor non-universalities in the lepton sector will be reserved for future study. The only relevant trilinear term in the slepton sector is the tau trilinear coupling $A_{\tau}$, which, along with $A_{t}$ and $A_{b}$ and all other parameters, we will take to be real.

\subsection{Higgs spectrum}

The physical Higgs spectrum of the MSSM consists of two neutral scalar bosons $h$ and $H$, one neutral pseudoscalar $A$, and a pair of charged Higgs bosons $H^{ \pm}$. At tree level, the full spectrum is determined by only two real parameters: the mass of the pseudoscalar Higgs, $M_{A}$, and the ratio of the two vacuum expectation values, $\tan \beta=t_{\beta}=v_{u} / v_{d}$, with $v_{u}^{2}+v_{d}^{2}=v^{2}=174^{2} \mathrm{GeV}^{2}$. In the so-called decoupling limit, $M_{A}^{2} \gg M_{W}^{2}$, the masses of the Higgs bosons, $A, H$ and $H^{ \pm}$, are

$$
M_{H}^{2} \simeq M_{A}^{2}, \quad M_{H^{ \pm}}^{2} \simeq M_{A}^{2}+M_{W}^{2} .
$$

In this limit, the mass of the lightest Higgs $h$ is given at tree level by

$$
M_{h}^{2} \simeq M_{Z}^{2} \cos 2 \beta
$$

As is well known, moderate or large values of $\tan \beta$ and large 1-loop corrections are required to lift $M_{h}$ up to phenomenologically viable values. Moreover, at large $\tan \beta$, the sbottom and stau 1-loop corrections can lower $M_{h}$ by a few $\mathrm{GeV}$, which cannot be neglected given the current Higgs mass precision data. The dominant stop, sbottom, and stau loop contributions for large $\tan \beta$ read

$$
\begin{aligned}
\Delta M_{h}^{2} \simeq & \frac{3}{4 \pi^{2}} \frac{m_{t}^{4}}{v^{2}}\left[\log \left(\frac{m_{\tilde{t}}^{2}}{m_{t}^{2}}\right)+\frac{X_{t}^{2}}{m_{\tilde{t}}^{2}}-\frac{X_{t}^{4}}{12 m_{\tilde{t}}^{4}}\right] \\
& -\frac{3}{48 \pi^{2}} \frac{m_{b}^{4}}{v^{2}} \frac{t_{\beta}^{4}}{\left(1+\epsilon_{b} t_{\beta}\right)^{4}} \frac{\mu^{4}}{m_{\tilde{b}}^{4}}-\frac{1}{48 \pi^{2}} \frac{m_{\tau}^{4}}{v^{2}} \frac{t_{\beta}^{4}}{\left(1+\epsilon_{\ell} t_{\beta}\right)^{4}} \frac{\mu^{4}}{m_{\tilde{\tau}}^{4}},
\end{aligned}
$$

where $X_{t}=A_{t}-\mu / \tan \beta \approx A_{t}$ for large $\tan \beta$, and $m_{\tilde{t}}, m_{\tilde{b}}$ and $m_{\tilde{\tau}}$ are the average stop, sbottom, and stau masses, respectively. The stop loop corrections, reported in the first line of (2.5), are maximized for $A_{t} \simeq \sqrt{6} m_{\tilde{t}}$. The contributions from the sbottom and stau loops, in the second and third lines, always reduce the light Higgs mass and can be particularly important for large $\tan \beta$, large values of the Higgsino mass parameter, $\mu$, and light sbottom or stau masses [97]. The $\epsilon_{i}$ factors come from an all-order resummation of $\tan \beta$ enhanced corrections to the Higgs-fermion couplings and are discussed in detail in section 2.3 .

The couplings of the lightest Higgs to SM fermions and gauge bosons are mainly controlled by $\tan \beta$ and the angle $\alpha$ that diagonalizes the mass matrix of the two scalar Higgs bosons. If

$$
\alpha=\beta-\pi / 2,
$$

the couplings of $h$ are exactly SM-like. At the tree level, eq. (2.6) holds up to corrections of order $M_{Z}^{2} /\left(t_{\beta} M_{A}^{2}\right)$. Correspondingly, for large $\tan \beta$ and moderately heavy $M_{A}$, the 
couplings of $h$ are already SM-like to a good approximation. At 1-loop, eq. (2.6) gets corrected by an additional term $\sim \lambda_{7} v^{2} / M_{A}^{2}$, where $\lambda_{7}$ is a loop-induced quartic Higgs coupling that reads

$$
\begin{aligned}
\lambda_{7} \simeq & \frac{3}{96 \pi^{2}} \frac{m_{t}^{4}}{v^{4}} \frac{\mu A_{t}}{m_{\tilde{t}}^{2}}\left(\frac{A_{t}^{2}}{m_{\tilde{t}}^{2}}-6\right) \\
& +\frac{3}{96 \pi^{2}} \frac{m_{b}^{4}}{v^{4}} \frac{t_{\beta}^{4}}{\left(1+\epsilon_{b} t_{\beta}\right)^{4}} \frac{\mu^{3} A_{b}}{m_{\tilde{b}}^{4}}+\frac{1}{96 \pi^{2}} \frac{m_{\tau}^{4}}{v^{4}} \frac{t_{\beta}^{4}}{\left(1+\epsilon_{\ell} t_{\beta}\right)^{4}} \frac{\mu^{3} A_{\tau}}{m_{\tilde{\tau}}^{4}} .
\end{aligned}
$$

If $\lambda_{7}$ is sizable, corrections to the light Higgs couplings can become relevant, as discussed in the next section, and are constrained by the SM Higgs searches at the LHC.

\subsection{Higgs couplings to fermions}

At tree level, the MSSM Higgs sector is a 2 Higgs doublet model of type II, where only $H_{u}$ couples to right-handed up quarks and only $H_{d}$ couples to right-handed down quarks and leptons. The Yukawa interactions thus have the following form

$$
\mathcal{L}_{\text {Yuk }}=\left(y_{u}\right)_{i j} H_{u} \bar{Q}_{i} U_{j}+\left(y_{d}\right)_{i j} H_{d} \bar{Q}_{i} D_{j}+\left(y_{\ell}\right)_{i j} H_{d} \bar{L}_{i} E_{j}+\text { h.c. } .
$$

As a consequence, the couplings of the neutral Higgs bosons to fermions are flavor diagonal in the mass eigenstate basis. At the loop level, however, "wrong" Higgs couplings are generated and lead to potentially large threshold corrections to the masses of down type quarks and leptons [98-101] as well as CKM matrix elements [102]. They also significantly modify charged Higgs couplings to quarks $[103,104]$ and generate flavor changing neutral Higgs couplings [95, 96, 105-110]. All these effects become particularly relevant in the large $\tan \beta$ regime, where the inherent 1-loop suppression can be partly compensated. In the following, we analyze the form of the neutral and charged Higgs couplings with fermions in the phenomenologically motivated limit, $v^{2} \ll M_{\text {SUSY }}^{2}$ (see [110] for a discussion of the regime $\left.v^{2} \sim M_{\mathrm{SUSY}}^{2}\right)$. We consistently take into account the most generic MFV structure of the squark masses as discussed in section 2.1. In particular, we consider splittings between the first two and the third generation squarks in the left-handed as well as righthanded sectors.

Once the 1-loop corrections are taken into account and we have diagonalized the quark mass matrices, the couplings of the neutral Higgs mass eigenstates to fermions have the following generic form

$$
\begin{aligned}
\mathcal{L}_{\text {int }} \supset & \sum_{q, q^{\prime}} \frac{m_{q}}{\sqrt{2} v}\left(\bar{q}_{L}^{\prime} q_{R}\right)\left(\xi_{q^{\prime} q}^{h} h+\xi_{q^{\prime} q}^{H} H+i \xi_{q^{\prime} q}^{A} A\right) \\
& +\sum_{\ell} \frac{m_{\ell}}{\sqrt{2} v}\left(\bar{\ell}_{L} \ell_{R}\right)\left(\xi_{\ell}^{h} h+\xi_{\ell}^{H} H+i \xi_{\ell}^{A} A\right)+\text { h.c. },
\end{aligned}
$$

where we have neglected flavor changing couplings to leptons, which are not relevant for our analysis. Using the notation $\xi_{q q}^{i}=\xi_{q}^{i}$, and again for large $\tan \beta$, the flavor conserving 
couplings of the heavy scalar and pseudoscalar Higgses, normalized to their respective SM Yukawas, are

$$
\begin{aligned}
-\xi_{q}^{H} & \simeq \xi_{q}^{A} \simeq \frac{1}{t_{\beta}}-\epsilon_{q}, \quad \text { for } q=u, c, t, \\
\xi_{q}^{H} & \simeq \xi_{q}^{A} \simeq \frac{t_{\beta}}{1+\epsilon_{q} t_{\beta}}, \quad \text { for } q=d, s, b, \\
\xi_{\ell}^{H} & \simeq \xi_{\ell}^{A} \simeq \frac{t_{\beta}}{1+\epsilon_{\ell} t_{\beta}} .
\end{aligned}
$$

In the above expressions, $\tan \beta$-enhanced corrections to the couplings are resummed to all orders and the $\epsilon_{i}$ factors parametrize the loop induced "wrong" Higgs couplings. The exact form of each $\epsilon_{i}$ in terms of MSSM parameters is discussed at the end of this section. Since we assume the MSSM parameters to be $C P$ conserving, all $\epsilon_{i}$ parameters are real.

Among the flavor changing Higgs couplings only the coupling of a right handed bottom with a left-handed strange quark will be relevant in the following discussion. Normalized to the bottom Yukawa of the SM, we have

$$
\xi_{s b}^{H} \simeq \xi_{s b}^{A} \simeq \frac{\epsilon_{\mathrm{FC}} t_{\beta}^{2}}{\left(1+\epsilon_{b} t_{\beta}\right)\left(1+\epsilon_{0} t_{\beta}\right)} V_{t b} V_{t s}^{*},
$$

where $\epsilon_{0}$ is defined as $\epsilon_{0}=\epsilon_{b}-\epsilon_{\mathrm{FC}}$, and $\epsilon_{\mathrm{FC}}$ is discussed in detail below.

The couplings of the light Higgs boson, $h$, are exactly SM-like in the decoupling limit: $\xi_{q}^{h}=\xi_{\ell}^{h}=1$ and $\xi_{q^{\prime} q}^{h}=0$ for $q^{\prime} \neq q$. While non-standard effects in the couplings to up-type quarks are generically tiny even away from the decoupling limit, corrections to the couplings with down-type quarks and leptons decouple very slowly and can be relevant. We have

$$
\xi_{f}^{h}=-\frac{s_{\alpha}}{c_{\beta}} \frac{1-\epsilon_{f} / t_{\alpha}}{1+\epsilon_{f} t_{\beta}}, \quad \text { for } f=d, s, b, \ell .
$$

The couplings of the physical charged Higgs bosons to fermions can be written as

$$
\mathcal{L}_{\text {int }} \supset \sum_{q, q^{\prime}} \frac{m_{q}}{v}\left(\bar{q}_{L}^{\prime} q_{R}\right) \xi_{q^{\prime} q}^{ \pm} H^{ \pm}+\sum_{\ell} \frac{m_{\ell}}{v}\left(\bar{\nu}_{L}^{\ell} \ell_{R}\right) \xi_{\nu \ell}^{ \pm} H^{ \pm}+\text {h.c. } .
$$

For the couplings relevant to our analysis, we have

$$
\begin{aligned}
& \frac{\xi_{t b}^{ \pm}}{V_{t b}}=\frac{t_{\beta}}{1+\epsilon_{b} t_{\beta}}, \quad \frac{\xi_{u s}^{ \pm}}{V_{u s}}=\frac{t_{\beta}}{1+\epsilon_{s} t_{\beta}}, \\
& \frac{\xi_{u b}^{ \pm}}{V_{u b}}=\frac{\xi_{c b}^{ \pm}}{V_{c b}}=\frac{t_{\beta}}{1+\epsilon_{0} t_{\beta}}, \quad \xi_{\nu \ell}^{ \pm}=\frac{t_{\beta}}{1+\epsilon_{\ell} t_{\beta}}, \\
& \frac{\xi_{s t}^{ \pm}}{V_{t s}^{*}}=\frac{1}{t_{\beta}}-\epsilon_{0}^{\prime}+\epsilon_{\mathrm{FC}}^{\prime} \frac{\epsilon_{\mathrm{FC}} t_{\beta}}{1+\epsilon_{0} t_{\beta}},
\end{aligned}
$$

where $V_{i j}$ are CKM matrix elements and $\epsilon_{0}^{\prime}=\epsilon_{t}-\epsilon_{\mathrm{FC}}^{\prime}$. The parameter $\epsilon_{\mathrm{FC}}^{\prime}$ is the up-sector analogue of $\epsilon_{\mathrm{FC}}$. 
As already mentioned, the various $\epsilon$ factors in the above expressions parametrize loopinduced non-holomorphic Higgs couplings. They arise from Higgsino-squark loops, gluinosquark loops and wino-sfermion loops. We do not explicitly state the typically negligible contributions coming from bino-sfermion loops; however, they are included in our numerical analysis.

For the bottom quark, we can decompose $\epsilon_{b}=\epsilon_{b}^{\tilde{g}}+\epsilon_{b}^{\tilde{W}}+\epsilon_{b}^{\tilde{H}}$, where these contributions are

$$
\begin{aligned}
\epsilon_{b}^{\tilde{g}} & =\frac{\alpha_{s}}{4 \pi} \frac{8}{3} \mu M_{3} g\left(M_{3}^{2}, m_{Q_{3}}^{2}, m_{D_{3}}^{2}\right), \\
\epsilon_{b}^{\tilde{W}} & =-\frac{\alpha_{2}}{4 \pi} \frac{3}{2} \mu M_{2} g\left(\mu^{2}, M_{2}^{2}, m_{Q_{3}}^{2}\right), \\
\epsilon_{b}^{\tilde{H}} & =\frac{\alpha_{2}}{4 \pi} \frac{m_{t}^{2}}{2 M_{W}^{2}} \mu A_{t} g\left(\mu^{2}, m_{Q_{3}}^{2}, m_{U_{3}}^{2}\right) .
\end{aligned}
$$

The loop function $g$ is listed in the appendix, and has units of $(\mathrm{GeV})^{-2}$. Hence, the $\epsilon$ factors generally exhibit non-decoupling as the SUSY mass scale increases. In particular, rescaling all the SUSY mass parameters, i.e. the squark masses, gaugino masses, the Higgsino mass parameter and the trilinear coupling by a common, arbitrarily large factor, leaves the $\epsilon$ parameters invariant. For a degenerate SUSY spectrum with mass $\tilde{m}$, we obtain $g\left(\tilde{m}^{2}, \tilde{m}^{2}, \tilde{m}^{2}\right)=1 / 2 \tilde{m}^{2}$. Our sign convention is that the left-right mixing entries in the top and bottom squark mass matrices are given by $m_{t}\left(A_{t}-\mu \cot \beta\right)$ and $m_{b}\left(A_{b}-\mu \tan \beta\right)$, respectively. Furthermore, the gluino mass $M_{3}$ is always positive in our convention.

For the strange and down quarks, the Higgsino contribution is highly suppressed by small Yukawa couplings or CKM angles and only the gluino and wino loops are relevant: $\epsilon_{s, d}=\epsilon_{s, d}^{\tilde{g}}+\epsilon_{s, d}^{\tilde{W}}$, where the $\epsilon_{s, d}^{i}$ can be easily obtained from the corresponding $\epsilon_{b}^{i}$ expressions, replacing third generation squark masses with second or first generation squark masses.

For leptons, only the wino (and the bino) loops give contributions, and $\epsilon_{\ell}^{\tilde{W}}$ is given by $\epsilon_{b}^{\tilde{W}}$ with the sbottom masses replaced by the slepton masses.

In case of the top quark, analogous to the bottom quark, we consider the gluino, wino, and Higgsino contributions: $\epsilon_{t}=\epsilon_{t}^{\tilde{g}}+\epsilon_{t}^{\tilde{W}}+\epsilon_{t}^{\tilde{H}}$. The expressions for $\epsilon_{t}^{\tilde{g}}$ and $\epsilon_{t}^{\tilde{W}}$ are trivially obtained from the corresponding $\epsilon_{b}^{i}$ by replacing the relevant squark masses. The Higgsino contribution is explicitly given by

$$
\epsilon_{t}^{\tilde{H}}=-\frac{\alpha_{2}}{4 \pi} \frac{m_{b}^{2}}{2 M_{W}^{2}} \frac{t_{\beta}^{2}}{\left(1+\epsilon_{b} t_{\beta}\right)^{2}} \quad \mu A_{b} g\left(\mu^{2}, m_{Q_{3}}^{2}, m_{D_{3}}^{2}\right) .
$$

Here, $\epsilon_{t}^{\tilde{H}}$ is suppressed by the bottom quark mass and only becomes relevant for large values of $\tan \beta$.

The flavor changing couplings, $\epsilon_{\mathrm{FC}}$ and $\epsilon_{\mathrm{FC}}^{\prime}$, can be decomposed as

$$
\begin{aligned}
& \epsilon_{\mathrm{FC}}=\epsilon_{b}^{\tilde{H}}+\zeta \epsilon_{\mathrm{FC}}^{\tilde{g}}+\zeta \epsilon_{\mathrm{FC}}^{\tilde{W}}, \\
& \epsilon_{\mathrm{FC}}^{\prime}=\epsilon_{t}^{\tilde{H}}+(1-\zeta) \epsilon_{\mathrm{FC}}^{\prime \tilde{g}}+(1-\zeta) \epsilon_{\mathrm{FC}}^{\tilde{W}}
\end{aligned}
$$


with

$$
\begin{aligned}
\epsilon_{\mathrm{FC}}^{\tilde{g}} & =\frac{\alpha_{s}}{4 \pi} \frac{8}{3} \mu M_{\tilde{g}}\left(g\left(M_{3}^{2}, m_{Q_{3}}^{2}, m_{D_{3}}^{2}\right)-g\left(M_{3}^{2}, m_{Q^{2}}^{2}, m_{D_{3}}^{2}\right)\right), \\
\epsilon_{\mathrm{FC}}^{\tilde{W}} & =-\frac{\alpha_{2}}{4 \pi} \frac{3}{2} \mu M_{2}\left(g\left(\mu^{2}, M_{2}^{2}, m_{Q_{3}}^{2}\right)-g\left(\mu^{2}, M_{2}^{2}, m_{Q}^{2}\right)\right),
\end{aligned}
$$

and $\epsilon_{\mathrm{FC}}^{\prime \tilde{g}}$ is obtained from $\epsilon_{\mathrm{FC}}^{\tilde{g}}$ by replacing the right-handed sbottom mass, $m_{D_{3}}$, with the right-handed stop mass, $m_{U_{3}}$. The $\epsilon_{b}^{\tilde{H}}$ and $\epsilon_{t}^{\tilde{H}}$ expressions were already given above. Note that $m_{D_{3}}$ enters both loop functions in $(2.25)$ and hence, in general, $\epsilon_{\mathrm{FC}}^{\tilde{g}} \neq \epsilon_{b}^{\tilde{g}}-\epsilon_{s}^{\tilde{g}}$, in contrast to the case where all right-handed down squarks have the same mass, $m_{D_{3}}=m_{D}$. Clearly, a splitting between the third and the first two generations of left-handed squarks induces non-zero $\epsilon_{\mathrm{FC}}^{\tilde{g}}, \epsilon_{\mathrm{FC}}^{\tilde{W}}$ and/or $\epsilon_{\mathrm{FC}}^{\prime \tilde{g}}$.

As already described in section 2.1, $\zeta$ parametrizes the alignment of the left-handed squark mass with the quark masses. The case $\zeta=1$ corresponds to a $m_{Q}^{2}$ that is aligned in the up sector such that the mass splitting between the first two and the third generations leads to off-diagonal entries only in the down squark mass matrix. This in turn implies maximal flavor changing gaugino loop corrections to the Higgs-down quark couplings. The case $\zeta=0$ corresponds to alignment in the down sector, with no off-diagonal entries appearing in the down squark mass matrix. Generically, from RGE running, we expect $1 / 2<\zeta<1$.

\section{Higgs collider searches}

\subsection{SM-like $125 \mathrm{GeV}$ Higgs}

The LHC experiments, ATLAS and CMS, have recently discovered a new particle with a mass of about $125 \mathrm{GeV}[30,31]$. This discovery is based on results from SM Higgs searches in the $\gamma \gamma, Z Z$ and $W W$ channels. The observed signals indicate that the new particle is a boson with spin 0 or 2, and overall, they are in reasonable agreement with expectations for a SM Higgs. Other searches in the $\tau^{+} \tau^{-}$and $b b$ decay channels are also being pursued, but more statistics are needed in order to make conclusive statements.

The most visible feature of the extracted signal strength in all the different channels under study is an enhancement in the $\gamma \gamma$ decay rate in comparison to the SM rate. The decay rates into $W W$ and $Z Z$ gauge bosons are consistent with the SM values at the $1 \sigma$ level. The present experimental uncertainties in the signal strength in the various production and decay channels allow for many new physics alternatives. In particular, within supersymmetric extensions, it is possible to enhance or suppress the gluon fusion production with light stops, depending on the amount of mixing in the stop sector. It is also possible to suppress gluon fusion with light sbottoms that have sizable mixing driven by large values of $\mu \tan \beta$. In all cases, enhancement of gluon fusion implies a suppression of the $h \rightarrow \gamma \gamma$ decay rate, and vice-versa. The overall effective $g g \rightarrow h \rightarrow \gamma \gamma$ rate, however, is governed by the enhancement or suppression of the gluon fusion production cross section.

To achieve a net enhancement in the $h \rightarrow \gamma \gamma$ rate, uncorrelated with a simultaneous enhancement in the $h \rightarrow W W / Z Z$ rates coming from an enhanced gluon fusion production 
or reduced $h \rightarrow b \bar{b}$ partial width, the existence of new, light, charged colorless particles running in the loop is required. In the MSSM, the only two options are charginos, which only contribute for $\tan \beta \sim 1$ (disfavored by a $125 \mathrm{GeV}$ Higgs mass), and light staus with large mixing, i.e. large $\mu \tan \beta$. A detailed discussion of the possible deviations from SM values of the production and decay rates for a SM-like Higgs in the MSSM can be found in [39, 58]. Possible correlations with flavor observables have very recently been studied in [75].

While it is very interesting to investigate deviations from SM expectations in Higgs data that would point towards new SUSY particles within the reach of the LHC, we take a different approach in this work by assuming a Higgs boson with approximately SMlike properties. We concentrate on possible signatures of new physics that may appear in $B$ physics observables, direct non-SM Higgs searches and dark matter direct detection searches within the MSSM with MFV, while fulfilling the requirement of a $125 \mathrm{GeV}$ SMlike Higgs. In this way, we show indirect effects from SUSY particles in flavor and Higgs physics in regions of parameter space beyond the present reach of the LHC.

\subsection{Searches for heavy scalars and pseudoscalars}

Searches for the heavy neutral Higgs bosons of the MSSM have been performed in the $H / A \rightarrow b b$ and $H / A \rightarrow \tau^{+} \tau^{-}$channels both at the Tevatron [111-114] and the LHC [77-79, 115, 116].

Searches also exist for light charged Higgs bosons in top decays at both the Tevatron $[117,118]$ and the LHC [119-121]. For the MSSM scenarios considered in this work, however, the corresponding bounds are not competitive with the bounds from searches of the neutral Higgs bosons.

In the large $\tan \beta$ regime, the cross sections for the heavy scalar and pseudoscalar Higgses rescale according to

$$
\begin{aligned}
\sigma_{b b \rightarrow H} \simeq \sigma_{b b \rightarrow A} \simeq \sigma_{b b \rightarrow h}^{\mathrm{SM}} \times \frac{t_{\beta}^{2}}{\left(1+\epsilon_{b} t_{\beta}\right)^{2}}, \\
\sigma_{g g \rightarrow H} \simeq \sigma_{g g \rightarrow A} \simeq \sigma_{g g \rightarrow h}^{t t, \mathrm{SM}} \times\left(\frac{1}{t_{\beta}}-\epsilon_{t}\right)^{2}+\sigma_{g g \rightarrow h}^{t b, \mathrm{SM}} \times \frac{1-\epsilon_{t} t_{\beta}}{1+\epsilon_{b} t_{\beta}}+\sigma_{g g \rightarrow h}^{b b, \mathrm{SM}} \times \frac{t_{\beta}^{2}}{\left(1+\epsilon_{b} t_{\beta}\right)^{2}},
\end{aligned}
$$

evaluated at a common mass for all Higgs bosons. For large $\tan \beta$, the $\sigma_{b b \rightarrow H / A}$ production cross sections can dominate over gluon fusion. We use HIGLU [122] and bbh@nnlo [123] to compute the respective SM cross sections $\sigma_{g g \rightarrow h}^{i, \mathrm{SM}}$ and $\sigma_{b b \rightarrow h}^{\mathrm{SM}}$ at the LHC.

The most important decay modes of the heavy Higgs bosons are $H, A \rightarrow b b$ and $H, A \rightarrow \tau^{+} \tau^{-}$. The corresponding partial widths can be written as

$$
\begin{gathered}
\Gamma_{H b b} \simeq \Gamma_{A b b} \simeq \Gamma_{h b b}^{\mathrm{SM}} \times \frac{t_{\beta}^{2}}{\left(1+\epsilon_{b} t_{\beta}\right)^{2}}, \\
\Gamma_{H \tau \tau} \simeq \Gamma_{A \tau \tau} \simeq \Gamma_{h \tau \tau}^{\mathrm{SM}} \times \frac{t_{\beta}^{2}}{\left(1+\epsilon_{\tau} t_{\beta}\right)^{2}},
\end{gathered}
$$

where $\Gamma_{h f f}^{S M}$ are the corresponding decay widths of a Higgs boson with the same mass as $H$ and $A$ and with SM-like couplings to $b b$ and $\tau^{+} \tau^{-}$. In our numerical analysis, we compute $\Gamma_{h f f}^{\mathrm{SM}}$ using HDECAY [124]. 


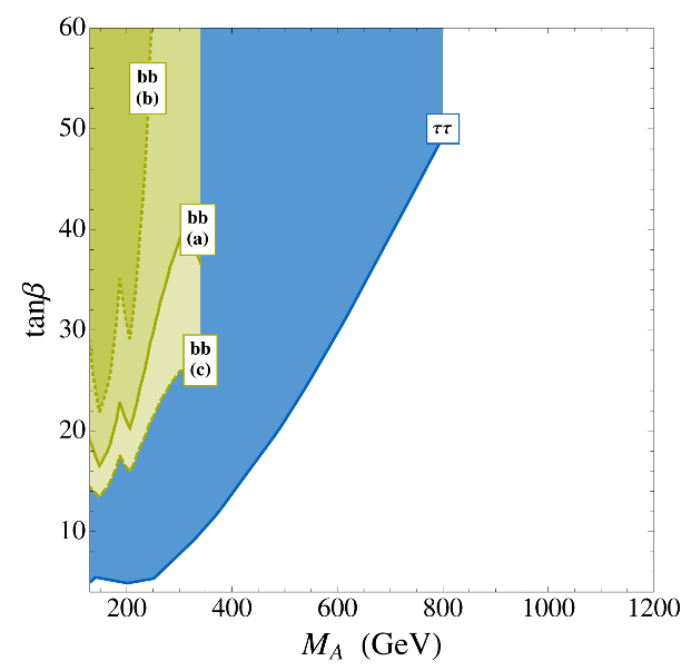

Figure 1. Constraints in the $M_{A}-\tan \beta$ plane from direct searches of the neutral MSSM Higgs bosons at CMS and ATLAS. The solid, dotted and dashed lines correspond to scenarios (a), (b), and (c) as defined in table 1 . The blue (green) regions are excluded by searches in the $\tau^{+} \tau^{-}(b b)$ channel.

\begin{tabular}{|c|ccccc|}
\hline Scenario & (a) & (b) & (c) & (d) & (e) \\
\hline \hline$\mu[\mathrm{TeV}]$ & 1 & 4 & -1.5 & 1 & -1.5 \\
$\operatorname{sign}\left(A_{t}\right)$ & + & + & + & - & - \\
\hline
\end{tabular}

Table 1. Illustrative MSSM scenarios discussed in the text. All sfermion masses are set to a common value $2 \mathrm{TeV}$, the gaugino masses to $6 M_{1}=3 M_{2}=M_{3}=1.5 \mathrm{TeV}$. The trilinear couplings $A_{t}=A_{b}=A_{\tau}$ are set such that the lightest Higgs mass is $M_{h}=125 \mathrm{GeV}$.

Note that the main dependence of the production cross sections and branching ratios is on $\tan \beta$ and the heavy Higgs masses. Dependence on other MSSM parameters enters only at the loop level through the $\tan \beta$ resummation factors $\epsilon_{i}$.

In our framework, the most important constraints come from the CMS bounds in the $\tau^{+} \tau^{-}$channel [77], which are available up to masses of $M_{A}=800 \mathrm{GeV}$ and the $b \bar{b}$ channel $[78,79]$ which cover heavy Higgs masses up to $M_{A}<350 \mathrm{GeV}$. Our estimates for the excluded regions from the $H / A \rightarrow b \bar{b}$ searches are shown in figure 1 in yellowgreen and labeled with $b b$. We set all sfermion masses to $2 \mathrm{TeV}$ and the gaugino masses to $6 M_{1}=3 M_{2}=M_{3}=1.5 \mathrm{TeV}$. The solid, dotted and dashed contours correspond to a Higgsino mass parameter $\mu=1 \mathrm{TeV}$ (scenario a), $4 \mathrm{TeV}$ (scenario b) and $-1.5 \mathrm{TeV}$ (scenario c), respectively. For every point in the $M_{A}-\tan \beta$ plane, the trilinear couplings $A_{t}=A_{b}=A_{\tau}$ are positive and chosen such that the lightest Higgs mass, computed using FeynHiggs [125], is $M_{h}=125 \mathrm{GeV} .^{2}$ The respective choices for $\mu$ lead to representative values for the $\tan \beta$ resummation factors of $\epsilon_{b}^{\tilde{g}}+\epsilon_{b}^{\tilde{W}} \simeq 3.3 \times 10^{-3}, 1.5 \times 10^{-2}$ and $-5.1 \times 10^{-3}$.

\footnotetext{
${ }^{2}$ The Higgs mass, $M_{h}$, is not a monotonic function in $A_{t}$ and for a given sign of $A_{t}$ there are typically two choices of $A_{t}$ that lead to $M_{h}=125 \mathrm{GeV}$. We always take the $A_{t}$ that is smaller in magnitude.
} 
As is well known, the bounds in the $M_{A}-\tan \beta$ plane coming from the $\tau^{+} \tau^{-}$channel are robust against variations of the MSSM parameters. Indeed, the dependence of the production cross section on $\epsilon_{b}$ is largely cancelled by the corresponding dependence of the $\operatorname{BR}\left(A, H \rightarrow \tau^{+} \tau^{-}\right)[126,127]$. In figure 1 , we therefore simply report in blue the $\tan \beta$ bounds obtained in [77] in the so-called $M_{h}^{\max }$ scenario. We checked explicitly that the constraints are largely independent of the scenarios in table 1 . We find that the constraints can only be weakened mildly for large $\tan \beta$ and $M_{A}$ if the MSSM parameters are such that $\epsilon_{b}$ is sizable and positive, as in scenario (b). ${ }^{3}$ We note however, that in the region with low $\tan \beta$, the bounds do depend to some extent on the SUSY spectrum, in particular the neutralino and chargino spectrum. Indeed, for low $\tan \beta$, the heavy scalar and pseudoscalar Higgs bosons can have sizable branching ratios in neutralinos or charginos if these decays are kinematically allowed. The $M_{h}^{\max }$ scenario considered in [77] contains light neutralinos with $M_{\chi_{1}} \simeq 95 \mathrm{GeV}$. For small $\tan \beta$, the obtained bounds from the searches in the $\tau^{+} \tau^{-}$ channel are therefore slightly weaker compared to scenarios with heavier neutralinos.

The CMS searches in the $b b$ channel $[78,79]$ are not yet competitive with the $\tau^{+} \tau^{-}$ searches, but might become important for large $M_{A}$ in the future [128]. Compared to the $\tau^{+} \tau^{-}$searches, the bounds coming from the $b b$ searches show a stronger dependence on the remaining MSSM parameters $[126,127]$. In particular, for large negative $\mu$, the bounds become significantly stronger, while for large positive $\mu$, the bounds can be weakened considerably. Note that for large negative $\mu$ and large $\tan \beta$, however, constraints from vacuum stability and perturbativity of the bottom Yukawa have to be taken into account.

Since the theoretical precision of the light Higgs mass prediction in the MSSM allows shifts of a few $\mathrm{GeV}$, we checked the extent to which the $H / A \rightarrow \tau^{+} \tau^{-}$and $H / A \rightarrow b \bar{b}$ bounds depend on the exact value of the Higgs mass assumed in our analysis, $M_{h}=$ $125 \mathrm{GeV}$. We find that varying the light Higgs mass in the range $122 \mathrm{GeV}<M_{h}<128 \mathrm{GeV}$ does not change the constraints from $H$ and $A$ searches significantly.

\section{Vacuum stability}

Independent of experimental searches, large values of $\mu$ can be constrained based on vacuum stability considerations, particularly if $\tan \beta$ is also large. Indeed, large values of $\mu \tan \beta$ can lead to charge and color breaking minima in the scalar potential of the MSSM [129].

The trilinear couplings of the up-type Higgs, $H_{u}$, with sbottoms, $\tilde{b}_{L}$ and $\tilde{b}_{R}$, and staus, $\tilde{\tau}_{L}$ and $\tilde{\tau}_{R}$, are controlled by

$$
\mathcal{L} \supset \frac{m_{\tau}}{v} \frac{\mu \tan \beta}{1+\epsilon_{\tau} \tan \beta}\left(H_{u}^{0} \tilde{\tau}_{L}^{*} \tilde{\tau}_{R}\right)+\frac{m_{b}}{v} \frac{\mu \tan \beta}{1+\epsilon_{b} \tan \beta}\left(H_{u}^{0} \tilde{b}_{L}^{*} \tilde{b}_{R}\right)+\text { h.c. }
$$

For trilinear couplings that are large compared to the sbottom or stau masses, minima with non-zero vevs for the sbottom and/or stau fields can arise in addition to the standard

\footnotetext{
${ }^{3}$ The CMS results in the $\tau^{+} \tau^{-}$channel are only available as constraints in the $M_{A}-\tan \beta$ plane for the $M_{h}^{\max }$ scenario. We translate these constraints into bounds on the corresponding $\sigma \times \mathrm{BR}$ and then reinterpret the cross section bounds as constraints in the $M_{A}-\tan \beta$ plane for various choices of the other MSSM parameters summarized in table 1. We assume constant efficiencies throughout this procedure.
} 
electroweak minimum. If these minima are deeper than the electroweak minimum, the electroweak minimum becomes unstable and can decay. The corresponding regions of parameter space are only viable as long as the lifetime of the electroweak minimum is longer than the age of the universe. This corresponds to requiring that the bounce action, $B$, of the tunneling process is $B \gtrsim 400[130,131]$.

Our phenomenological flavor analysis is largely independent of the values of the stau masses, and non-zero stau vevs can always be avoided if $m_{\tilde{\tau}_{L}}$ and $m_{\tilde{\tau}_{R}}$ are large enough. Nonetheless, we will consider a scenario where the squark and slepton masses are the same order and thus include both sbottoms and staus in the following analysis.

Starting with the MSSM scalar potential, we restrict ourselves to terms that contain only the up-type Higgs, sbottoms, and staus, which are the degrees of freedom most relevant for large $\mu \tan \beta$. We consider three cases: (i) only terms with the up-type Higgs and staus, (ii) only up-type Higgs and sbottoms, and (iii) up-type Higgs, staus and sbottoms simultaneously. In each case, we search for additional minima in field space and estimate the bounce action for tunneling from the electroweak minimum into the deepest minimum of the potential. In the end, we apply the strongest of the three bounds.

For each case, the second vacuum generally has separately nearly degenerate stau vevs and nearly equal sbottom vevs. In the case of the sbottoms, this is expected from the $\mathrm{SU}(3) D$-terms in the scalar potential:

$$
\mathcal{L} \supset \frac{g_{s}^{2}}{6}\left(\left|\tilde{b}_{L}\right|^{2}-\left|\tilde{b}_{R}\right|^{2}\right)^{2}
$$

We can clearly see that at least for the 3-dimensional parameter space in case (ii), deviations from equality of the squark/slepton fields along the path chosen to compute the action would come at the expense of large contributions from the $D$ terms. Therefore, to obtain an analytical estimate for the bounce action, we consider a straight path in field space connecting the electroweak minimum and the charge and/or color breaking minimum.

We then approximate the potential along the straight line by a triangle and use the analytical expressions in [132] to calculate the bounce action. We construct the triangle such that for a few chosen parameter points, the obtained bounce action agrees approximately with the bounce action from the analytic expression of the potential solved numerically by a standard overshoot/undershoot method. We further crosschecked our results with CosmoTransitions [133] taking into account the up-type Higgs, the down-type Higgs, the sbottoms, and the staus. Overall, we find good agreement with our approximate analytical approach.

The constraints thus derived in the $\mu-\tan \beta$ plane are shown in figure 2 . We fix the SUSY masses as in the scenarios considered above, namely we assume degenerate sfermion masses with $\tilde{m}=2 \mathrm{TeV}$ and gaugino masses with $6 M_{1}=3 M_{2}=M_{3}=1.5 \mathrm{TeV}$. The trilinear couplings we set to $A_{t}=2 \mathrm{TeV}$ in the left and to $A_{t}=-2 \mathrm{TeV}$ in the right plot. In the white region, the electroweak minimum is the deepest minimum in the potential and therefore absolutely stable. In the light red (light gray) region, a charge (and possibly color) breaking minimum exists that is deeper than the electroweak minimum, but the electroweak minimum has a lifetime longer than the age of the universe. In the dark 

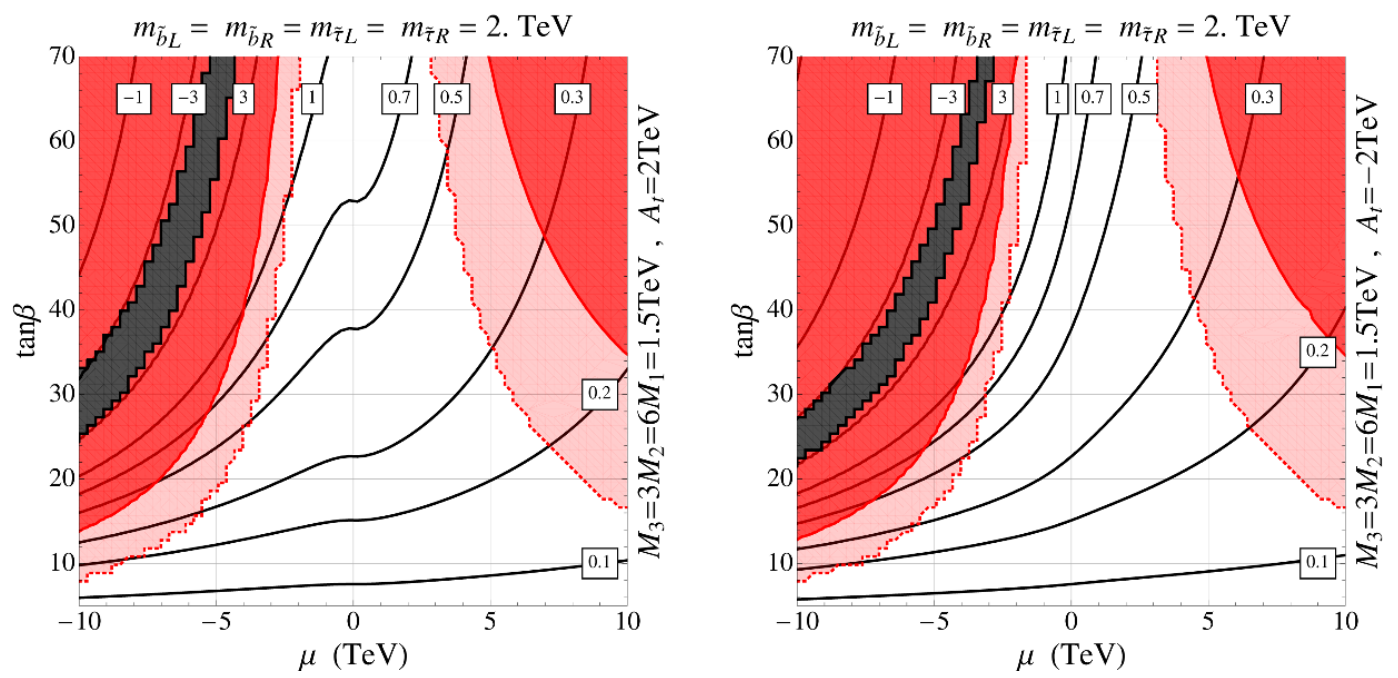

Figure 2. Constraints from vacuum stability in the $\mu-\tan \beta$ plane. We set the sbottom and stau soft masses to $2 \mathrm{TeV}$ and the gaugino masses to $6 M_{1}=3 M_{2}=M_{3}=1.5 \mathrm{TeV}$. In the left (right) plot, the trilinear coupling of the stops is $A_{t}=2 \mathrm{TeV}\left(A_{t}=-2 \mathrm{TeV}\right)$. The labeled contours show the values of the bottom Yukawa coupling. In the light red (light gray) regions, a charge and color breaking vacuum exists that is deeper than the electroweak breaking vacuum, but the electroweak vacuum has a lifetime that is longer than the age of the universe. In the dark red (gray) regions, the electroweak vacuum is not stable on cosmological time scales. Finally, in the black regions, one of the sbottoms becomes tachyonic.

red (gray) region the lifetime of the electroweak minimum is shorter than the age of the universe. Finally, in the black region, one of the sbottoms is tachyonic.

The solid lines labeled in the plots show contours of constant bottom Yukawa couplings in the $\mu$-tan $\beta$ plane. For large and negative $\mu \tan \beta$, close to the region where one of the sbottoms becomes tachyonic, the bottom Yukawa coupling becomes non perturbatively large.

We observe that large negative values for $\mu$ are strongly constrained by the requirement of vacuum stability. This is because the $\tan \beta$ resummation factor, $\epsilon_{b}$, in (4.1) is linearly proportional to $\mu$. It increases the trilinear coupling of the up-type Higgs with sbottoms for negative values of $\mu$ and can lead to a deep second minimum mainly in the field direction of the sbottoms. In particular, we find that values of $\mu \tan \beta$ negative and large enough that the bottom Yukawa changes its sign (the parameter space in the upper left corner of the plot beyond the region excluded by tachyonic sbottoms) are excluded by the requirement of vacuum stability. For positive values of $\mu$, the coupling of the up-type Higgs with sbottoms is reduced while its coupling with staus is slightly enhanced by the $\epsilon_{\tau}$ term. In this region of parameter space, constraints come typically from a second minimum in the stau direction. Positive values for $\mu$ are less constrained than negative ones, and the allowed region for $\mu$ can be extended above $\mu>10 \mathrm{TeV}$ for sufficiently heavy staus.

The viable regions of parameter space can be enlarged slightly when we allow for a splitting between the masses of the left- and right-handed sbottoms and staus. Nevertheless, we do not find any regions of parameter space where both vacuum stability and 
$\epsilon_{b} \tan \beta<-1$ (which flips the sign of the tree level bottom Yukawa and hence changes the typical sign of the SUSY contribution to $B$ observables) can be achieved simultaneously. In the end, we see that the scenarios discussed in the previous section are all compatible with bounds from vacuum stability.

\section{$5 \quad B$ physics observables}

Flavor observables play a crucial role in determining viable regions of parameter space of SUSY models. This is true both under the MFV assumption [89-91, 134-140] and if new sources of flavor violation are allowed [141-150].

Of particular importance in the MFV setup are rare $B$ decays that are helicity suppressed in the SM, because SUSY contributions to these decays can be enhanced by $\tan \beta$ factors. Interesting processes include the tree level decay $B \rightarrow \tau \nu$, the purely leptonic decay $B_{s} \rightarrow \mu^{+} \mu^{-}$, and the radiative decay $B \rightarrow X_{s} \gamma$. Additional constraints on the SUSY parameter space can be also derived from the $(g-2)$ of the muon. The $(g-2)_{\mu}$ bound becomes particularly important if sleptons are only moderately heavy, which is a scenario that we do not consider here.

\section{$5.1 \quad B \rightarrow \tau \nu, B \rightarrow D^{(*)} \tau \nu$ and $K \rightarrow \mu \nu$}

The decay $B \rightarrow \tau \nu$ is a sensitive probe of extended Higgs sectors as it can be modified by charged Higgs exchanges at tree level [151]. The most important inputs for the SM prediction are the CKM element $\left|V_{u b}\right|$ and the $B$ meson decay constant. Using the PDG value $\left|V_{u b}\right|=(3.89 \pm 0.44) \times 10^{-3}$ [152], a conservative average over direct determinations from inclusive and exclusive semi-leptonic $B$ decays, and an average of recent precise lattice determinations of the decay constant $f_{B}=(190 \pm 4) \mathrm{MeV}$ [153-156], we find

$$
\mathrm{BR}(B \rightarrow \tau \nu)_{\mathrm{SM}}=(0.97 \pm 0.22) \times 10^{-4} .
$$

While previous experimental data gave values for the branching ratio more than $2 \sigma$ above the SM prediction, a recent result from Belle [81] has a much lower central value. An average of all the available data from BaBar $[82,157]$ and Belle $[81,158]$ gives

$$
\mathrm{BR}(B \rightarrow \tau \nu)_{\exp }=(1.16 \pm 0.22) \times 10^{-4} .
$$

This value is in very good agreement with the SM but still leaves room for NP contributions.

Closely related decay modes that are also sensitive to charged Higgs effects are the $B \rightarrow$ $D \tau \nu$ and $B \rightarrow D^{*} \tau \nu$ decays [159-163]. While predictions of the corresponding branching ratios suffer from large hadronic uncertainties coming from the $B \rightarrow D$ and $B \rightarrow D^{*}$ form factors, the ratios $\mathrm{BR}(B \rightarrow D \tau \nu) / \mathrm{BR}(B \rightarrow D \ell \nu)$ and $\mathrm{BR}\left(B \rightarrow D^{*} \tau \nu\right) / \mathrm{BR}(B \rightarrow$ $\left.D^{*} \ell \nu\right)$, where $\ell=e$ or $\mu$, can be predicted with reasonable accuracy in the SM $[162,164]$. Interestingly, recent results from BaBar [165] on these ratios are around $2 \sigma$ above the SM predictions in both decay modes. Older results from Belle [166] give similar central values but with much larger uncertainties.

Another interesting observable in this context is $R_{\mu 23}$ [167] that probes the tree level charged Higgs exchange in the $K \rightarrow \mu \nu$ decay. The much smaller sensitivity of $K \rightarrow \mu \nu$ 
to charged Higgs effects compared to the $B$ decays is compensated by its extremely high experimental precision and the excellent control on theoretical uncertainties giving [167]

$$
R_{\mu 23}=0.999 \pm 0.007 \text {. }
$$

All the mentioned tree level decays depend in similar ways on possible new physics contributions in the MSSM with MFV. Defining

$$
X_{B(K)}^{2}=\frac{1}{M_{H^{ \pm}}^{2}} \frac{t_{\beta}^{2}}{\left(1+\epsilon_{0(s)} t_{\beta}\right)\left(1+\epsilon_{\ell} t_{\beta}\right)},
$$

we can write

$$
\begin{aligned}
R_{B \tau \nu} & =\frac{\mathrm{BR}(B \rightarrow \tau \nu)}{\mathrm{BR}(B \rightarrow \tau \nu)_{\mathrm{SM}}} \\
& =\left(1-m_{B^{+}}^{2} X_{B}^{2}\right)^{2} \\
R_{D \tau \nu} & =\frac{\mathrm{BR}(B \rightarrow D \tau \nu)}{\mathrm{BR}(B \rightarrow D \tau \nu)_{\mathrm{SM}}} \\
& =\left(1-1.5 m_{\tau} m_{b} X_{B}^{2}+1.0 m_{\tau}^{2} m_{b}^{2} X_{B}^{4}\right), \\
R_{D^{*} \tau \nu} & =\frac{\mathrm{BR}\left(B \rightarrow D^{*} \tau \nu\right)}{\mathrm{BR}\left(B \rightarrow D^{*} \tau \nu\right)_{\mathrm{SM}}} \\
& =\left(1-0.12 m_{\tau} m_{b} X_{B}^{2}+0.05 m_{\tau}^{2} m_{b}^{2} X_{B}^{4}\right), \\
R_{\mu 23} & =\frac{\mathrm{BR}(K \rightarrow \mu \nu)}{\mathrm{BR}(K \rightarrow \mu \nu)_{\mathrm{SM}}} \\
& =\left(1-m_{K^{+}}^{2} X_{K}^{2}\right)
\end{aligned}
$$

In figure 3 we show these ratios as function of $X_{B, K}=\sqrt{\left|X_{B, K}^{2}\right|}$ both for positive $X_{i}^{2}$ (solid lines) and negative $X_{i}^{2}$ (dotted lines) in comparison with the experimental $1 \sigma$ and $2 \sigma$ ranges (dashed bands) from (5.1)-(5.3) and [162]-[165]. Here, positive $X_{i}^{2}$ illustrates destructive interference with the SM, while negative $X_{i}^{2}$ illustrates constructive interference with the SM.

We observe that agreement of theory and experiment in all three $B$ observables is impossible to achieve. In particular the tensions in $B \rightarrow D \tau \nu$ and $B \rightarrow D^{*} \tau \nu$ cannot be addressed in the context of the MSSM with MFV, but require more radical approaches [168-172].

Considering MSSM contributions to $K \rightarrow \mu \nu$ and $B \rightarrow \tau \nu$, we observe that generally, $X_{B}$ and $X_{K}$ are equal to a good approximation. The only way to induce a difference is through a splitting between the right-handed strange squark mass and the right-handed bottom squark mass which enter the corresponding $\epsilon$ factors in the definitions of $X_{B}$ and $X_{K}$. As discussed in section 2.1, such a splitting is compatible with the MFV ansatz for the squark spectrum as long as $\tan \beta$ is large enough that $y_{b}$ effects cannot be neglected. However, we find that even for a large mass splitting $X_{B} \simeq X_{K}$ holds, except for regions of parameter space with large and negative $\mu$, such that $\epsilon_{0(s)} \tan \beta \sim \mathcal{O}(-1)$. Such regions 


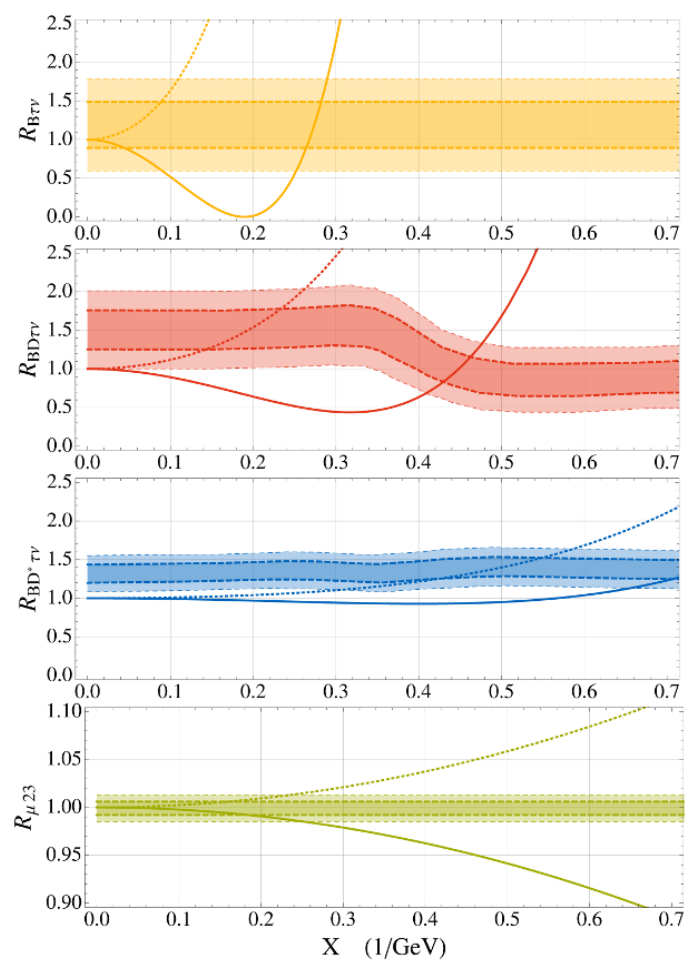

Figure 3. Branching ratios of the decays $B \rightarrow \tau \nu, B \rightarrow D \tau \nu, B \rightarrow D^{*} \tau \nu$, and $K \rightarrow \mu \nu$ as functions of $X_{B}, X_{K}=\sqrt{\left|X_{B, K}^{2}\right|}$ as appropriate and which are defined in the main text. The dashed bands show the $1 \sigma$ and $2 \sigma$ experimental ranges. The solid (dotted) lines are the theory predictions for positive (negative) $X^{2}$ giving destructive (constructive) interference with the SM amplitude.

of parameter space are strongly constrained by perturbativity of the bottom Yukawa and vacuum stability considerations, as discussed in section 4 . If $X_{B} \simeq X_{K}$, then the $B \rightarrow \tau \nu$ decay gives stronger constraints than $K \rightarrow \mu \nu{ }^{4}$

In the following, we therefore concentrate on the constraint from $B \rightarrow \tau \nu$ on the MSSM parameter space. Apart from corners of parameter space with very large and negative $\epsilon_{0} \tan \beta<-1$, the charged Higgs contribution interferes destructively with the $\mathrm{SM}\left(X_{B}^{2}>0\right)$, and leads to constraints in the $M_{H^{ \pm}}-\tan \beta$ plane. These constraints depend on other SUSY parameters only through the loop-induced $\tan \beta$ resummation factors $\epsilon_{i}$ and are therefore robust in large parts of parameter space.

The yellow lines in the left plot of figure 4 show the $B \rightarrow \tau \nu$ constraints in the $M_{A^{-}}$ $\tan \beta$ plane corresponding to the 3 choices of MSSM parameters (a), (b), and (c) given in table 1 and already discussed in section 3.2. For comparison, the constraint from direct searches in the $\tau^{+} \tau^{-}$channel is also shown in gray. There are also a narrow strips of small Higgs masses and large $\tan \beta$ where the NP contribution to the $B \rightarrow \tau \nu$ amplitude is twice as large as the SM contribution. This in turn implies that this region of parameters is in

\footnotetext{
${ }^{4}$ For the special range $0.25 \lesssim X_{B}, X_{K} \lesssim 0.30$ and $\epsilon_{0} \tan \beta>-1$, the $K \rightarrow \mu \nu$ constraint is stronger than $B \rightarrow \tau \nu$, but this parameter region is excluded by direct searches as discussed in the main text.
} 

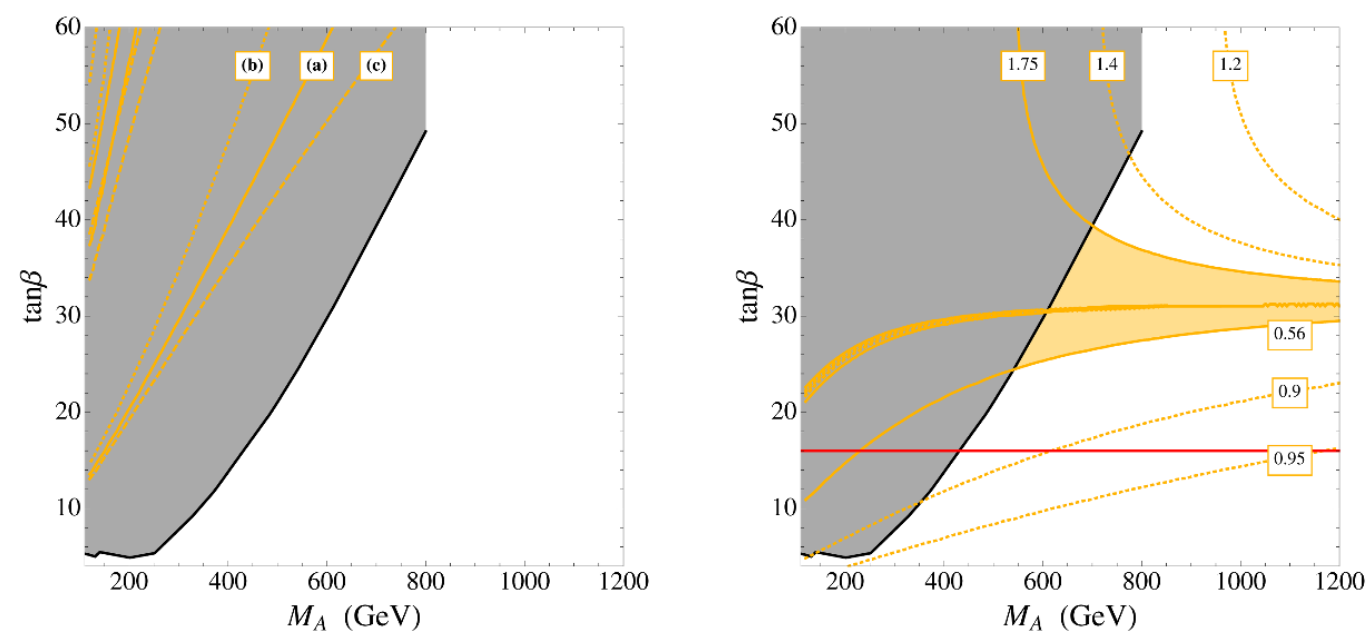

Figure 4. Constraints in the $M_{A}$-tan $\beta$ plane from the tree level $B \rightarrow \tau \nu$ decay. The constraint from direct heavy Higgs searches is also shown in gray. The yellow solid, dotted and dashed contours in the left plot correspond to scenarios (a), (b), and (c) defined in table 1. The right plot shows a scenario with $\mu=-8 \mathrm{TeV}$, leading to a large negative $\epsilon_{0}$ such that the charged Higgs contribution interferes constructively with the SM in the region with $\tan \beta \gtrsim 30$. The labeled contours indicate values for $R_{B \tau \nu}$. Above the red horizontal line, the electroweak vacuum has a lifetime shorter than the age of the universe.

principle allowed by the experimental data on $B \rightarrow \tau \nu$. It is in strong tension, however, with the results from $B \rightarrow D \tau \nu, B \rightarrow D^{*} \tau \nu$, and $K \rightarrow \mu \nu$ and furthermore is excluded by direct searches.

The dependence of the $B \rightarrow \tau \nu$ constraints on the $\tan \beta$ resummation factors is stronger than the one of the direct searches in the $\tau^{+} \tau^{-}$channel, especially for large values of $\tan \beta$. For large values of $\tan \beta$ and a positive (negative) value of $\epsilon_{0}$ the constraint can be weakened (strengthened) considerably. As $\epsilon_{0}$ does not depend on $A_{t}$, the constraint from $B \rightarrow \tau \nu$ is to a large extent insensitive to the exact value of the light Higgs mass. Constraints from direct MSSM Higgs searches are generically stronger for $M_{A}<800 \mathrm{GeV}$. While the latest results from direct MSSM Higgs searches in the $\tau^{+} \tau^{-}$channel at the LHC end at $M_{A}=800 \mathrm{GeV}$, obviously no such restriction exists for the $B \rightarrow \tau \nu$ constraints. Only very large values of $\tan \beta \gtrsim 60$, however, are typically probed by $B \rightarrow \tau \nu$ for such large heavy Higgs masses.

In corners of parameter space with very large negative $\mu$, we can have $\epsilon_{0} \tan \beta<-1$ for values of $\tan \beta$ that are not extremely large and when the bottom Yukawa is perturbative [140]. Such a situation is shown in the right plot of figure 4 , where $\mu=-8 \mathrm{TeV}$ and the resulting $\epsilon_{0} \simeq-0.03$. For $\tan \beta \lesssim 30$, the charged Higgs still interferes destructively with the SM. For larger values of $\tan \beta \gtrsim 30$, however, the sign of $X_{B}^{2}$ flips, the interference becomes constructive, and the branching ratio is always enhanced. This behavior can be seen from the values of $R_{B \tau \nu}$ indicated with the dotted contours in the right plot of figure 4 .

Note that vacuum stability requirements, however, strongly constrain very large and negative values of $\mu$. As discussed in section 4, we do not find viable regions of parameter space where the bottom Yukawa has a negative sign with respect to the SM one, i.e. with 
$\epsilon_{b} \tan \beta<-1$. For $B \rightarrow \tau \nu$, the relevant parameter combination is $\epsilon_{0} \tan \beta$. The horizontal red line in the right plot of figure 4 marks the upper bound on $\tan \beta$ in the scenario with $\mu=-8 \mathrm{TeV}$, such that the electroweak vacuum remains stable on timescales of the age of the universe. Therefore, we see that $\epsilon_{0} \tan \beta<-1$ is also excluded by vacuum stability considerations. This conclusion holds beyond the discussed $\mu=-8 \mathrm{TeV}$ example.

\section{$5.2 \quad B_{s} \rightarrow \mu^{+} \mu^{-}$}

The $B_{s} \rightarrow \mu^{+} \mu^{-}$decay is a flavor changing neutral current process and correspondingly only induced at the loop level, both in the SM and the MSSM. In the SM, $B_{s} \rightarrow \mu^{+} \mu^{-}$is also helicity suppressed by the muon mass, resulting in a tiny SM prediction, at the level of $10^{-9}$. Using the recently given precise value for the $B_{s}$ meson decay constant $f_{B_{s}}=$ $(227 \pm 4) \mathrm{MeV}$ [156] which is an average of several lattice determinations [153-155], and taking into account the effect of the large width difference in the $B_{s}$ meson system [173, 174], we have the branching ratio extracted from an untagged rate as [175] (see also [176])

$$
\mathrm{BR}\left(B_{s} \rightarrow \mu^{+} \mu^{-}\right)_{\mathrm{SM}}=(3.32 \pm 0.17) \times 10^{-9} .
$$

Experimental searches for that decay have been carried out at D0 [177] and CDF [178], and are ongoing at ATLAS [179], CMS [180], and LHCb [80, 181]. Very recently, the LHCb collaboration reported first evidence for the $B_{s} \rightarrow \mu^{+} \mu^{-}$decay [80]. LHCb finds for the branching ratio the following value

$$
\operatorname{BR}\left(B_{s} \rightarrow \mu^{+} \mu^{-}\right)_{\exp }=\left(3.2_{-1.2}^{+1.4}{ }_{-0.3}^{+0.5}\right) \times 10^{-9},
$$

and gives the following two sided $95 \%$ C.L. bound

$$
1.1 \times 10^{-9}<\operatorname{BR}\left(B_{s} \rightarrow \mu^{+} \mu^{-}\right)_{\exp }<6.4 \times 10^{-9} .
$$

We use this bound in our analysis. Note that the upper bound in (5.11) is considerably weaker than the official combination of the previous LHCb result [181] with the ATLAS and CMS bounds [182].

For large values of $\tan \beta$, order of magnitude enhancements of the $\operatorname{BR}\left(B_{s} \rightarrow \mu^{+} \mu^{-}\right)$ are possible in the MSSM $[106,183]$. In the large $\tan \beta$ limit, the CP averaged branching ratio in the MFV MSSM can be written to a good approximation as

$$
R_{B_{s} \mu \mu}=\frac{\operatorname{BR}\left(B_{s} \rightarrow \mu^{+} \mu^{-}\right)}{\operatorname{BR}\left(B_{s} \rightarrow \mu^{+} \mu^{-}\right)_{\mathrm{SM}}} \simeq|\mathcal{A}|^{2}+|1-\mathcal{A}|^{2} .
$$

The MSSM contribution $\mathcal{A}$ is dominated by so-called Higgs penguins, i.e. the exchange of the heavy scalar $H$ and pseudoscalar $A$ with their 1-loop induced flavor changing $b \rightarrow s$ couplings, that are parametrized by $\epsilon_{\mathrm{FC}}$ given in (2.13). We find

$$
\mathcal{A}=\frac{4 \pi}{\alpha_{2}} \frac{m_{B_{s}}^{2}}{4 M_{A}^{2}} \frac{\epsilon_{\mathrm{FC}} t_{\beta}^{3}}{\left(1+\epsilon_{b} t_{\beta}\right)\left(1+\epsilon_{0} t_{\beta}\right)\left(1+\epsilon_{\ell} t_{\beta}\right)} \frac{1}{Y_{0}} .
$$

The SM loop function $Y_{0}$ depends on the top mass and is approximately $Y_{0} \simeq 0.96$. Note that the MSSM contributions to $B_{s} \rightarrow \mu^{+} \mu^{-}$do not decouple with the scale of the SUSY 


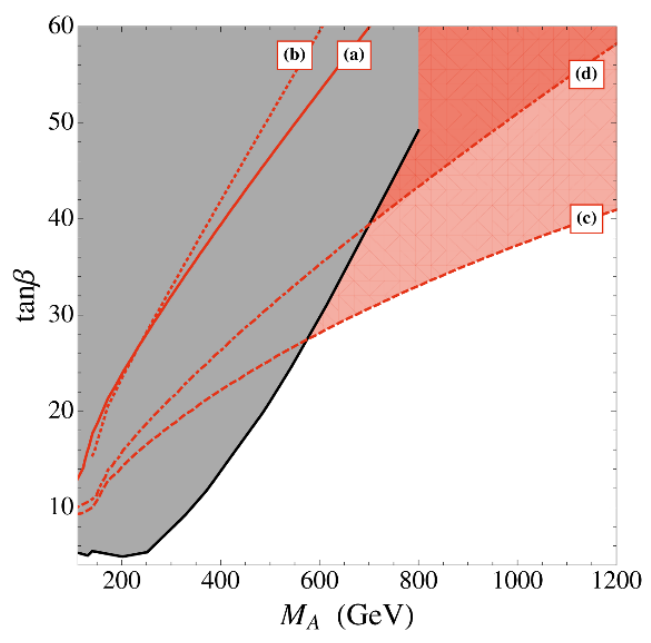

Figure 5. Constraints in the $M_{A}-\tan \beta$ plane from the $B_{s} \rightarrow \mu^{+} \mu^{-}$decay. The red solid, dotted, dashed and dash-dotted contours correspond to scenarios (a), (b), (c) and (d), as described in the text. The gray region is excluded by direct searches of MSSM Higgs bosons in the $H / A \rightarrow \tau^{+} \tau^{-}$ channel.

particles, but with the masses of the heavy scalar and pseudoscalar Higgs bosons $M_{H}^{2} \simeq$ $M_{A}^{2}$. Due to the strong enhancement by $\tan ^{3} \beta$, the large $\tan \beta$ regime of the MSSM is highly constrained by the current experimental results on $\operatorname{BR}\left(B_{s} \rightarrow \mu^{+} \mu^{-}\right)$. We remark, however, that $\epsilon_{\mathrm{FC}}$ in the numerator of (5.13) is a sum of several terms (see (2.23)) each of which depend strongly on several MSSM parameters. In addition, cancellations among the different terms can occur in certain regions of parameter space, rendering the $B_{s} \rightarrow \mu^{+} \mu^{-}$ constraint very model dependent, even in the restrictive framework of MFV. Additional contributions to $B_{s} \rightarrow \mu^{+} \mu^{-}$can arise from charged Higgs loops [187]. They interfere destructively with the SM contribution and scale as $(\tan \beta)^{2} / M_{H^{ \pm}}^{2}$. Typically, their effect is considerably smaller compared to the SUSY contribution in (5.13).

We stress that there is a simple mathematical lower bound of $R_{B_{s} \mu \mu}=1 / 2$ in (5.12) that is saturated for $\mathcal{A}=1 / 2$. In this case, the SUSY contribution partially cancels the SM amplitude, but simultaneously generates a non-interfering piece that cannot be canceled. This lower limit provides a significant threshold for experiments searching for $\operatorname{BR}\left(B_{s} \rightarrow\right.$ $\left.\mu^{+} \mu^{-}\right)$: not only is the SM branching fraction a meaningful value to test experimentally, but the potential observation of the branching fraction below one half of the SM value would strongly indicate NP and imply departure from the MSSM with MFV. Note that the current $2 \sigma$ lower bound from $\mathrm{LHCb}$ on the branching ratio is below $1 / 2$ of the SM value and therefore does not lead to constraints in our framework, yet.

In figure 5 , we show the constraints from $B_{s} \rightarrow \mu^{+} \mu^{-}$in the $M_{A}-\tan \beta$ plane. The red solid, dotted and dashed contours correspond to scenarios (a), (b), and (c) of table 1. The dash-dotted contour corresponds to scenario (d), with all MSSM parameters as for the solid contour, but with a negative sign for the trilinear coupling. For comparison, the constraints from direct searches are again shown in gray. As expected, we observe a very strong dependence of the $B_{s} \rightarrow \mu^{+} \mu^{-}$bounds on the choices of the remaining 
MSSM parameters, particularly the sign of $\mu A_{t}$. Note that in the considered scenarios, we assume degenerate squarks such that the only term entering $\epsilon_{\mathrm{FC}}$ is from the irreducible Higgsino loop contribution, $\epsilon_{b}^{\tilde{H}}$, whose sign is dictated by $\mu A_{t}$. For positive (negative) $\mu A_{t}$ the NP contribution interferes destructively (constructively) with the SM amplitude. Since the lower bound on $\mathrm{BR}\left(B_{s} \rightarrow \mu^{+} \mu^{-}\right)$from $\mathrm{LHCb}$ is still below half of the SM value, destructively interfering NP is much less constrained than constructively interfering NP.

The plots of figure 6 show in red the constraints from $B_{s} \rightarrow \mu^{+} \mu^{-}$in the plane of the third generation squark masses and the Higgsino mass parameter $\mu$. The gray horizontal band corresponds to the constraint from direct searches of charginos at LEP that exclude $|\mu| \lesssim 100 \mathrm{GeV}[184,185]$. In these plots, we fix $M_{A}=800 \mathrm{GeV}, \tan \beta=45$ (fully compatible with the $B \rightarrow \tau \nu$ constraint and not yet constrained by direct searches), and gaugino masses with $6 M_{1}=3 M_{2}=M_{3}=1.5 \mathrm{TeV}$. As in all the other plots, we vary the trilinear couplings $A_{t}=A_{b}=A_{\tau}$ throughout the plot such that the lightest Higgs mass is $M_{h}=125 \mathrm{GeV}$. The values for $A_{t}$ are indicated in the plots by the vertical dotted contours. The two plots correspond to positive and negative values of the $A$-terms. In the gray region in the lower left corners of the plots, the sbottom loop corrections to the lightest Higgs mass become so large that the lightest Higgs mass is always below $M_{h}<125 \mathrm{GeV}$ for any value of $A_{t}$, taking into account a $3 \mathrm{GeV}$ theory uncertainty. We checked that varying the light Higgs mass between $122 \mathrm{GeV}<M_{h}<128 \mathrm{GeV}$ can change the values of $A_{t}$ by around $25 \%$ in each direction and therefore can affect the constraints derived from $B_{s} \rightarrow \mu^{+} \mu^{-}$at a quantitative level. However, the qualitative picture of the constraints and the interplay of the SUSY contributions to $B_{s} \rightarrow \mu^{+} \mu^{-}$, as discussed below, are unaffected by this variation.

The solid contours are obtained under the assumption that the masses of the first two generation squarks are equal to the third generation, while for the dashed and dotted contours we assume the first two generations to be heavier by $50 \%$. For the dashed contours, we assume the splitting for the left-handed squarks to be fully aligned in the up-sector, such that gaugino-squark loops also contribute to $\epsilon_{\mathrm{FC}}$ with $\zeta=1$ (see (2.23) and (2.25)). We set $\zeta=0.5$ for the dotted contours, such that only half of the squark mass splitting induces flavor violation in the down-sector. For negative $A_{t}$, the obtained bounds show a strong dependence on the value of $\zeta$. The $\operatorname{BR}\left(B_{s} \rightarrow \mu^{+} \mu^{-}\right)$bounds in figure 6 clearly display the non-decoupling behavior mentioned above. Due to this non-decoupling, the $\operatorname{BR}\left(B_{s} \rightarrow \mu^{+} \mu^{-}\right)$results can constrain SUSY parameter space in regions that are beyond the current and expected future reach of direct searches.

A crucial element of our analysis is the viability of the cancellation of the SUSY contribution to the $B_{s} \rightarrow \mu^{+} \mu^{-}$branching ratio. This cancellation is driven by the presence of $\epsilon_{\mathrm{FC}}$ in (5.13), which is schematically given in (2.23) and its various contributions are detailed in (2.21), (2.25) and (2.26). First, in the following discussion, we neglect the wino contribution given by (2.26), which is generally smaller than the gluino contribution. This is due to the smallness of $M_{2}$ and $\alpha$ in (2.26) compared to $M_{3}$ and $\alpha_{s}$ in (2.25) (of course, our numerical analysis always includes the wino contribution). Since each SUSY contribution is proportional to $\mu$, we see that switching the sign of $\mu$ changes the relative sign between the SUSY and SM amplitudes. Furthermore, by switching the sign of $A_{t}$, between the left and right panels of figure 6 , we change the relative sign between the gluino 

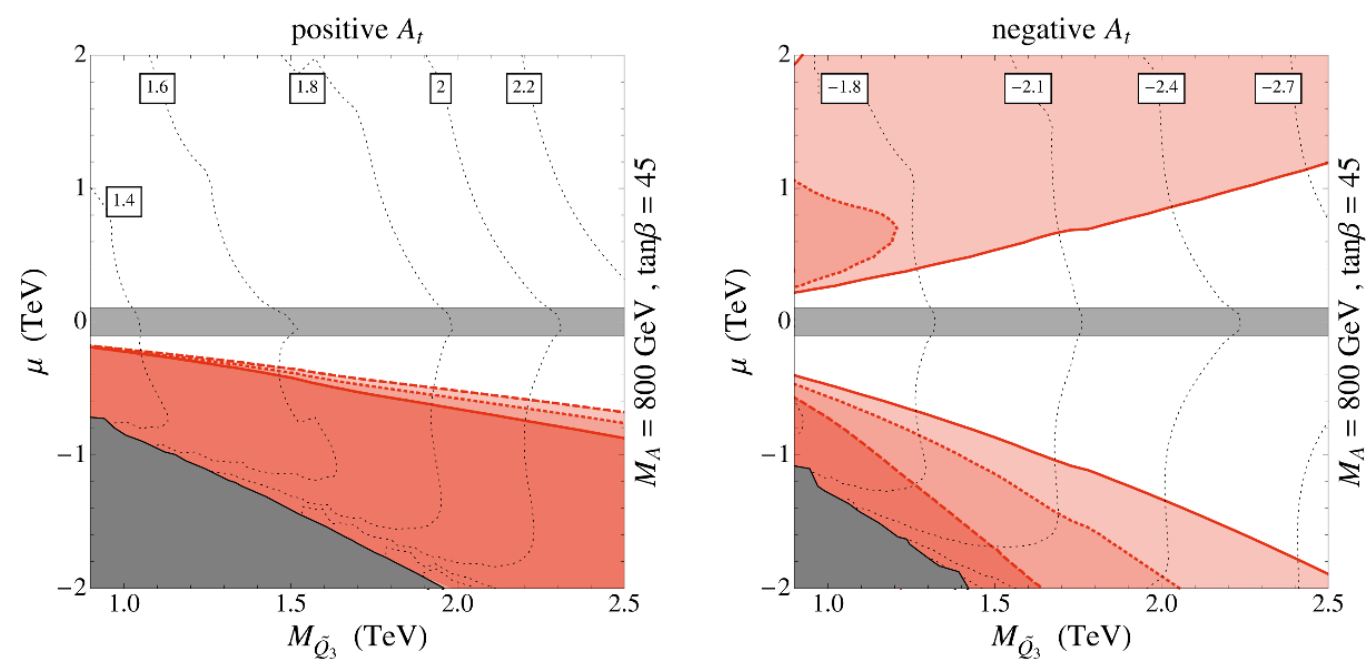

Figure 6. Constraints in the $m_{Q_{3}}-\mu$ plane from the $B_{s} \rightarrow \mu^{+} \mu^{-}$decay, with fixed $M_{3}=3 M_{2}=$ $6 M_{1}=1.5 \mathrm{TeV}, M_{A}=800 \mathrm{GeV}$ and $\tan \beta=45$. The solid bounded regions correspond to a degenerate squark spectrum. The dashed and dotted bounded regions correspond to choosing the first two squark generations $50 \%$ heavier than the third generation squark masses, with an alignment of $\zeta=1$ and $\zeta=0.5$, respectively. The gray horizontal band corresponds to the constraint from direct searches of charginos at LEP. The vertical dotted lines show contours of constant $A_{t}$ such that $M_{h}=125 \mathrm{GeV}$. In the gray regions in the lower left corners, the lightest Higgs mass is always below $M_{h}<125 \mathrm{GeV}$, taking into account a $3 \mathrm{GeV}$ theory uncertainty.

contribution and the Higgsino contribution. Thus, for a particular choice of $\operatorname{sign}\left(A_{t}\right)$ and $\operatorname{sign}(\mu)$, we can exploit a cancellation between the gluino vs. Higgsino loop, diminishing the magnitude of the SUSY contribution, and a second cancellation between the overall SUSY contribution and the SM amplitude. In particular, even if the magnitude of the SUSY contribution is by itself larger than the SM contribution, we can exercise the second cancellation where the SUSY amplitude overshoots the SM one.

These cancellations are clearly in effect in the left and right panels of figure 6 . We first focus on the regions bounded by solid lines, which correspond to degenerate squark masses. This implies that the SUSY contribution dominantly arises from $\epsilon_{b}^{\tilde{H}}$ in $(2.21)$. In the upper half of the left panel corresponding to positive $A_{t}$ and positive $\mu$, the SUSY contribution cancels with the SM contribution and always leads to a $\operatorname{BR}\left(B_{s} \rightarrow \mu^{+} \mu^{-}\right)$ below the current bound. In the lower half of the left panel, with positive $A_{t}$ and negative $\mu$, the Higgsino contribution adds constructively with the SM contribution, leading to significant constraints. In the upper half of the right panel, the Higgsino contribution also adds constructively with the SM, leading again to a bound. This bound is less stringent compared to the positive $A_{t}$ and negative $\mu$ case, because for positive $\mu$, the $\epsilon_{b}$ and $\epsilon_{0}$ terms in (5.13) lead to a suppression of the SUSY amplitude. Finally, in the lower half of the right panel, with negative $A_{t}$ and negative $\mu$, the Higgsino contribution interferes destructively with the SM. The constraint is non-vanishing, however, because for negative $\mu$, the $\tan \beta$ resummation factors, given in (5.13), enhance the SUSY amplitude such that it can be more than twice as large as the SM amplitude. 


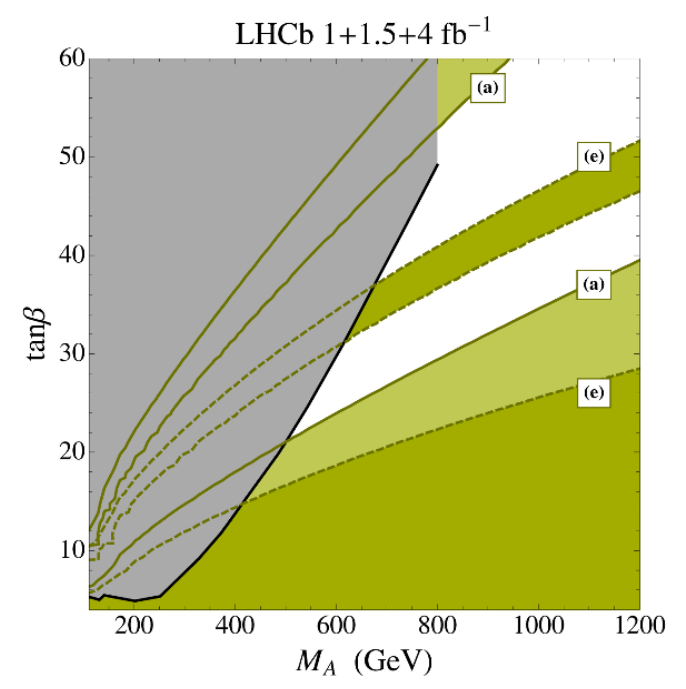

Figure 7. The $M_{A^{-}} \tan \beta$ plane in view of projected constraints from the $\operatorname{BR}\left(B_{s} \rightarrow \mu^{+} \mu^{-}\right)$, assuming a future $\pm 0.5 \times 10^{-9}$ uncertainty in the measurement with the SM prediction as the central value. The green shaded regions between and below the solid and dashed contours correspond to values for $\tan \beta$ and $M_{A}$ allowed in scenarios (a) and (e), as defined in table 1. The gray region is excluded by current direct searches of MSSM Higgs bosons in the $H / A \rightarrow \tau^{+} \tau^{-}$channel.

When we include squark splitting, we further strengthen the SUSY contribution for positive $A_{t}$, because the gluino and Higgsino contributions add constructively. Hence the overall SUSY+SM interference is more restricted. The bounds due to this splitting in the masses are shown by the regions enclosed by the dashed and dotted lines in figure 6 . For negative $A_{t}$, shown in the right panel, the gluino contribution partially cancels the Higgsino contribution, leading to a weaker constraint. The effect of the gluino contributions decreases for larger gluino mass, $M_{3}$.

In tandem, the complementary views provided by the different panels of figures 5 and 6 clearly demonstrate that certain choices of SUSY parameters relax the constraints considerably. For example, with $M_{A}=800 \mathrm{GeV}$ and $\tan \beta=45$, the region of parameter space with positive $\mu$ and positive $A_{t}$ is robustly unconstrained from the $B_{s} \rightarrow \mu^{+} \mu^{-}$ limit. Moving from top to bottom along a constant $A_{t}$ contour in the left plot of figure 6 corresponds to a rapid coverage of the $\tan \beta$ vs. $M_{A}$ plane from the (b) to (a) to (c) exclusion regions.

Regions of parameter space with destructive interference between SM and SUSY amplitudes (i.e. the regions with positive $\mu A_{t}$ ) will be constrained significantly if a lower bound of $\operatorname{BR}\left(B_{s} \rightarrow \mu^{+} \mu^{-}\right)$above half of the SM prediction is established in the future. We illustrate this in the plots of figures 7 and 8 , which assume a measurement of $\operatorname{BR}\left(B_{s} \rightarrow \mu^{+} \mu^{-}\right)$ at the SM expectation as a central value with an experimental uncertainty of $\pm 0.5 \times 10^{-9}$. Such a precision is expected to be achieved by $\mathrm{LHCb}$ at the end of the $13 \mathrm{TeV}$ run with a combined analysis of $1 \mathrm{fb}^{-1}$ of $7 \mathrm{TeV}$ data, $1.5 \mathrm{fb}^{-1}$ of $8 \mathrm{TeV}$ data, and $4 \mathrm{fb}^{-1}$ of $13 \mathrm{TeV}$ data [186]. The plots in figures 7 and 8 show in green the regions in the $M_{A^{-}} \tan \beta$ and $m_{Q_{3}}-\mu$ planes that are allowed by the expected results on the $B_{s} \rightarrow \mu^{+} \mu^{-}$decay. As shown 

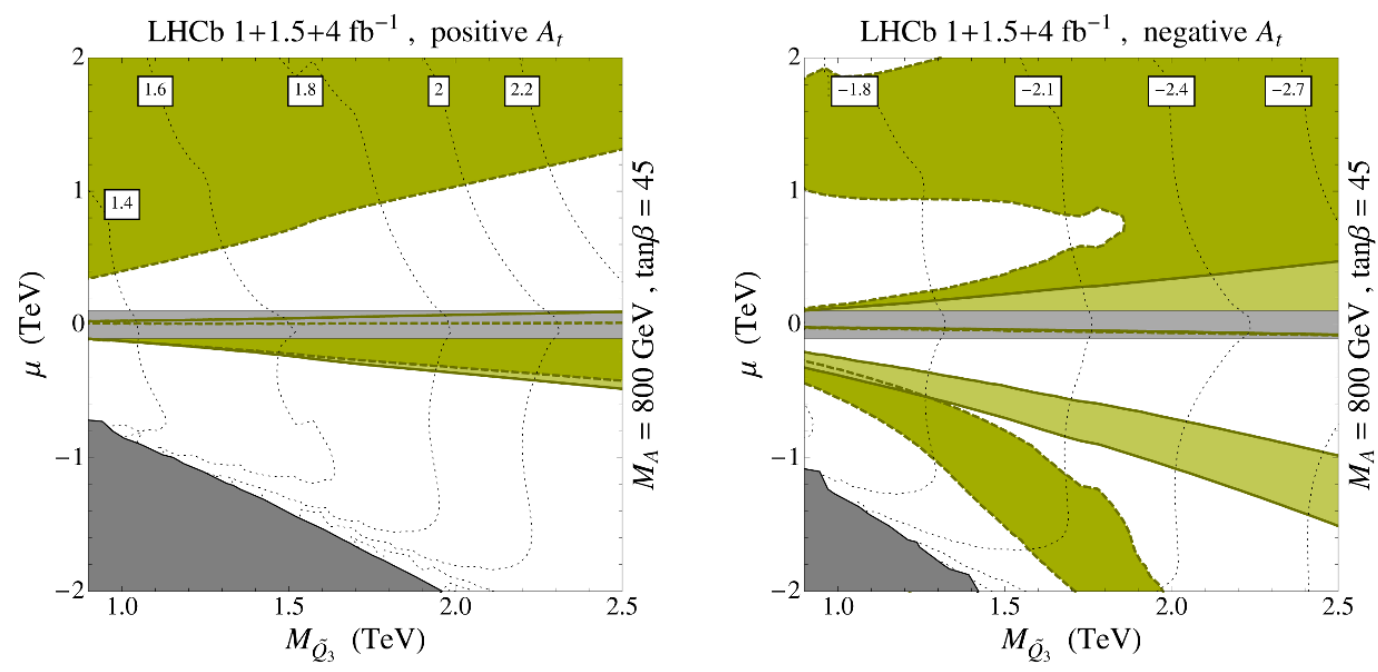

Figure 8. The $m_{Q_{3}}-\mu$ plane in view of projected constraints from the $\operatorname{BR}\left(B_{s} \rightarrow \mu^{+} \mu^{-}\right)$, assuming a future $\pm 0.5 \times 10^{-9}$ uncertainty in the measurement with the SM prediction as the central value. We fixed $M_{3}=3 M_{2}=6 M_{1}=1.5 \mathrm{TeV}, M_{A}=800 \mathrm{GeV}$ and $\tan \beta=45$. The green shaded regions between the solid contours correspond to values for $m_{Q_{3}}$ and $\mu$ allowed for a degenerate squark spectrum. The green shaded regions between and above the dashed contours are allowed if the first two squark generations are $50 \%$ heavier than the third generation squark masses, with an alignment of $\zeta=1$. The gray horizontal band corresponds to the constraint from direct searches of charginos at LEP. The vertical dotted lines show contours of constant $A_{t}$ such that $M_{h}=125 \mathrm{GeV}$. In the gray regions in the lower left corners, the lightest Higgs mass is always below $M_{h}<125 \mathrm{GeV}$, taking into account a $3 \mathrm{GeV}$ theory uncertainty.

in figure 7, apart from the allowed regions with large $M_{A}$ and small $\tan \beta$, there are also strips with large $M_{A}$ and large $\tan \beta$ where the expected bounds from $B_{s} \rightarrow \mu^{+} \mu^{-}$can be avoided. In these regions, the SUSY amplitude has approximately the same size as the SM amplitude but is opposite in sign. According to (5.12), this leads to a branching ratio close to the SM prediction.

For the example parameter point with $M_{A}=800 \mathrm{GeV}$ and $\tan \beta=45$, the projected lower bound on $\operatorname{BR}\left(B_{s} \rightarrow \mu^{+} \mu^{-}\right)$leads to very strong constraints in the $m_{Q_{3}}-\mu$ plane for positive $\mu A_{t}$. Indeed, for $M_{A}=800 \mathrm{GeV}$ and $\tan \beta=45$, and given the assumed experimental precision, charged Higgs loop contributions to $B_{s} \rightarrow \mu^{+} \mu^{-}$already lead to a non-negligible suppression [187], leaving hardly any room for destructively interfering SUSY contributions. Only if the SUSY contribution is so large that $\mathcal{A} \simeq-1$ does the parameter space open up again. The corresponding regions that are excluded by the assumed lower bound are clearly visible in the white region of the upper half of the left plot and the upper white region in the lower half of the right plot in figure 8 .

\section{$5.3 \quad B \rightarrow X_{s} \gamma$}

The loop induced $B \rightarrow X_{s} \gamma$ decay is also highly sensitive to NP effects coming from SUSY particles. The NNLO SM prediction for the branching ratio reads $[188]$ (see also $[189,190]$ )

$$
\operatorname{BR}\left(B \rightarrow X_{s} \gamma\right)_{\mathrm{SM}}=(3.15 \pm 0.23) \times 10^{-4} .
$$


On the experimental side, BaBar recently presented updated results for the branching ratio [83]. Including this, the new world average reads [191]

$$
\operatorname{BR}\left(B \rightarrow X_{s} \gamma\right)_{\exp }=(3.43 \pm 0.22) \times 10^{-4},
$$

which is slightly lower than the previous world average and is in very good agreement with the SM prediction. In the MSSM with minimal flavor violation and no new sources of $C P$ violation, the branching ratio can be written as [192]

$$
\begin{aligned}
R_{b s \gamma} & =\frac{\operatorname{BR}\left(B \rightarrow X_{s} \gamma\right)}{\operatorname{BR}\left(B \rightarrow X_{s} \gamma\right)_{\mathrm{SM}}} \\
& \simeq 1-2.55 C_{7}^{\mathrm{NP}}-0.61 C_{8}^{\mathrm{NP}}+0.74 C_{7}^{\mathrm{NP}} C_{8}^{\mathrm{NP}}+1.57\left(C_{7}^{\mathrm{NP}}\right)^{2}+0.11\left(C_{8}^{\mathrm{NP}}\right)^{2}
\end{aligned}
$$

where $C_{7,8}^{\mathrm{NP}}$ are the NP contributions to the magnetic and chromo-magnetic $b \rightarrow s \gamma$ operators evaluated at the scale $160 \mathrm{GeV}$.

Apart from the $B \rightarrow X_{s} \gamma$ decay, the modifications of the Wilson coefficients $C_{7}$ and $C_{8}$ also enter predictions of observables in the $B \rightarrow K^{*} \ell^{+} \ell^{-}$decay. In our MSSM setup with minimal flavor and $C P$ violation, we only have real NP contributions to $C_{7}$ and $C_{8}$. In this framework, the experimental data on $B \rightarrow K^{*} \ell^{+} \ell^{-}$does not put additional restrictions, once the bounds from $\mathrm{BR}\left(B \rightarrow X_{s} \gamma\right)$ are taken into account $[175,193]$. Therefore, we focus only on the $B \rightarrow X_{s} \gamma$ decay.

The SUSY contributions to $C_{7,8}^{\mathrm{NP}}$ come from charged Higgs-top loops, neutral Higgsbottom loops, Higgsino-stop loops, and gaugino-squark loops. As with the Higgs-fermion couplings, we take into account the most generic MFV structure of the squark masses and consistently consider splittings between the first two and the third generation squarks in the left-handed as well as the right handed sector. The resulting dominant MSSM contributions to $C_{7,8}$ read

$$
\begin{aligned}
C_{7,8}^{H}= & \left(\frac{1-\epsilon_{0}^{\prime} t_{\beta}}{1+\epsilon_{b} t_{\beta}}+\frac{\epsilon_{\mathrm{FC}}^{\prime} \epsilon_{\mathrm{FC}} t_{\beta}^{2}}{\left(1+\epsilon_{b} t_{\beta}\right)\left(1+\epsilon_{0} t_{\beta}\right)}\right) \frac{m_{t}^{2}}{2 M_{H^{ \pm}}^{2}} h_{7,8}\left(r_{t}\right)+\frac{\epsilon_{\mathrm{FC}} t_{\beta}^{3}}{\left(1+\epsilon_{b} t_{\beta}\right)^{2}\left(1+\epsilon_{0} t_{\beta}\right)} \frac{m_{b}^{2}}{2 M_{A}^{2}} z_{7,8}, \\
C_{7,8}^{\tilde{\tilde{H}}}= & -\frac{t_{\beta}}{1+\epsilon_{b} t_{\beta}} \frac{m_{t}^{2}}{2} A_{t} \mu f_{7,8}^{\tilde{H}}\left(m_{Q_{3}}^{2}, m_{U_{3}}^{2}, \mu^{2}\right), \\
\frac{g_{2}^{2}}{g_{3}^{2}} C_{7,8}^{\tilde{g}}= & \frac{t_{\beta}}{1+\epsilon_{0} t_{\beta}} M_{W}^{2} \mu M_{3} \zeta\left(f_{7,8}^{\tilde{g}}\left(m_{Q}^{2}, m_{D_{3}}^{2}, M_{3}^{2}\right)-f_{7,8}^{\tilde{g}}\left(m_{Q_{3}}^{2}, m_{D_{3}}^{2}, M_{3}^{2}\right)\right) \\
& -\frac{\epsilon_{\mathrm{FC}} t_{\beta}^{2}}{\left(1+\epsilon_{b} t_{\beta}\right)\left(1+\epsilon_{0} t_{\beta}\right)} M_{W}^{2} \mu M_{3} f_{7,8}^{\tilde{g}}\left(m_{Q_{3}}^{2}, m_{D_{3}}^{2}, M_{3}^{2}\right) \\
C_{7,8}^{\tilde{W}=} & \frac{t_{\beta}}{1+\epsilon_{0} t_{\beta}} M_{W}^{2} \mu M_{2} \zeta\left(f_{7,8}^{\tilde{W}}\left(M_{2}^{2}, \mu^{2}, m_{Q}^{2}\right)-f_{7,8}^{\tilde{W}}\left(M_{2}^{2}, \mu^{2}, m_{Q_{3}}^{2}\right)\right) \\
& -\frac{\epsilon_{\mathrm{FC}} t_{\beta}^{2}}{\left(1+\epsilon_{b} t_{\beta}\right)\left(1+\epsilon_{0} t_{\beta}\right)} M_{W}^{2} \mu M_{2} f_{7,8}^{\tilde{W}_{1}}\left(M_{2}^{2}, \mu^{2}, m_{Q_{3}}^{2}\right) .
\end{aligned}
$$

The first term in (5.17) corresponds to contributions from a charged Higgs loop. The loop functions, $h_{7,8}$ depend on the ratio of the top mass and the charged Higgs mass, 
$r_{t}=m_{t}^{2} / M_{H^{ \pm}}^{2}$, and for $r_{t}=1$ are given by $h_{7}(1)=-7 / 18$ and $h_{8}(1)=-1 / 3$. Their full analytical expressions can be found in the appendix. The second term in (5.17) arises from neutral heavy Higgs loops. It is strongly suppressed by the bottom quark mass and is only important for very large $\tan \beta$. The loop functions, $z_{7,8}$, depend on the ratio of the bottom mass and the charged Higgs mass and since $m_{b}^{2} / M_{H^{ \pm}}^{2} \ll 1$, they are very well approximated by $z_{7}=-\frac{1}{18}$ and $z_{8}=\frac{1}{6}$.

Contributions from Higgsino-stop, gluino-down squark, and Wino-down squark loops are shown in (5.18), (5.19), and (5.20), respectively. We do not write the typically negligible bino contributions.

For a degenerate SUSY spectrum with mass $\tilde{m}$, the loop functions entering the Higgsino and gaugino contributions reduce to

$$
\begin{aligned}
f_{7}^{\tilde{H}} & \rightarrow \frac{5}{36} \frac{1}{\tilde{m}^{4}}, \quad f_{7}^{\tilde{g}} \rightarrow-\frac{2}{27} \frac{1}{\tilde{m}^{4}}, \quad f_{7}^{\tilde{W}} \rightarrow-\frac{7}{24} \frac{1}{\tilde{m}^{4}}, \\
f_{8}^{\tilde{H}} & \rightarrow \frac{1}{12} \frac{1}{\tilde{m}^{4}}, \quad f_{8}^{\tilde{g}} \rightarrow-\frac{5}{18} \frac{1}{\tilde{m}^{4}}, \quad f_{8}^{\tilde{W}} \rightarrow-\frac{1}{8} \frac{1}{\tilde{m}^{4}} .
\end{aligned}
$$

Their full analytical expressions are collected in the appendix. In contrast to the Higgs penguin contributions to $B_{s} \rightarrow \mu^{+} \mu-$, the SUSY loop contributions to $b \rightarrow s \gamma$ do decouple with the SUSY scale.

The first terms in (5.19) and (5.20) correspond to 1-loop flavor changing gaugino contributions. They vanish for $m_{Q_{3}}=m_{Q}$, i.e. if there is no splitting between the first two and the third generations of left-handed squark masses. In the presence of a splitting, the parameter $\zeta$ again parametrizes the alignment of the left-handed squark mass matrix. As mentioned before, if the splitting is generated by RGE running we expect $1 / 2<\zeta<1$. The second terms in (5.19) and (5.20) are formally 2-loop contributions but they can be relevant for large $\tan \beta$. They do not vanish for degenerate masses $[110,143]$.

Similarly to $B_{s} \rightarrow \mu^{+} \mu^{-}$, the MSSM contribution to $B \rightarrow X_{s} \gamma$ is a sum of several terms that depend sensitively on many parameters, particularly the signs of $\mu$ and $A_{t}$.

In figure 9, we show in orange the constraints from $B \rightarrow X_{s} \gamma$ in the $M_{A^{-}} \tan \beta$ plane obtained analogous to the $B_{s} \rightarrow \mu^{+} \mu^{-}$constraints discussed previously. The plots of figure 10 show the $B \rightarrow X_{s} \gamma$ constraints in the plane of the third generation squark masses and the Higgsino mass parameter $\mu$, again in complete analogy to the $B_{s} \rightarrow \mu^{+} \mu^{-}$ constraints.

We can again see the connection between the constraints in the $\tan \beta$ vs. $M_{A}$ plane, given in figure 9 , and the $\mu$ vs. $m_{Q_{3}}$ plane, given in figure 10 . The squark masses are fixed to $2 \mathrm{TeV}$ in figure 9 . This causes the stop-chargino contribution to be essentially negligible, and hence we are only constrained by the Higgs contribution in the low $M_{A}$ and large $\tan \beta$ regions. For heavy squarks and low $\tan \beta$, the bound on the charged Higgs mass is approximately independent of the other SUSY parameters and is given by $M_{H^{ \pm}} \gtrsim 300 \mathrm{GeV}$. For large $\tan \beta$, the resummation factors in (5.17) become relevant. The most important effect arises from the factors $\epsilon_{0}^{\prime}$ and $\epsilon_{b}$ in the first term in (5.17). For negative $\mu, \epsilon_{0}^{\prime}$ and $\epsilon_{b}$ are negative and therefore the bounds become stronger for larger $\tan \beta$ in scenario (c). For positive $\mu$ (scenarios $\mathrm{a}, \mathrm{b}$, and $\mathrm{d}$ ) instead, the bounds are relaxed for large $\tan \beta$. As the dominant gluino contribution to $\epsilon_{0}^{\prime}$ and $\epsilon_{b}$ grows with $\mu$ the $B \rightarrow X_{s} \gamma$ constraint is 


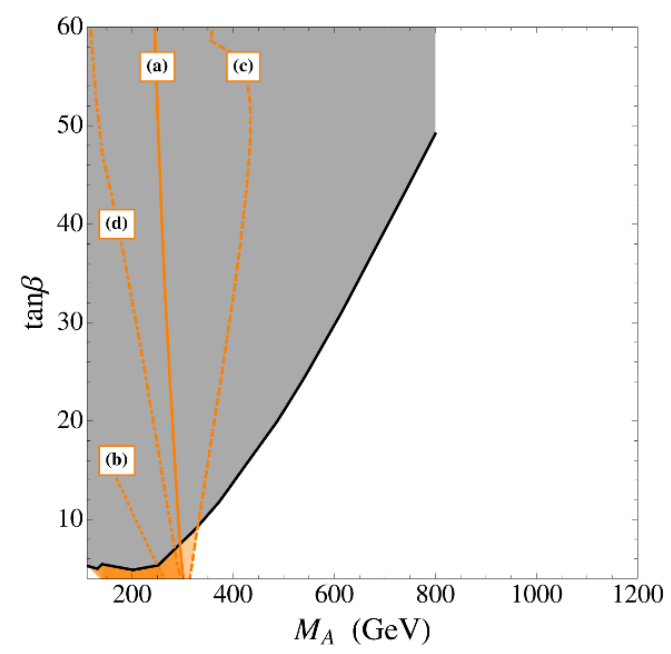

Figure 9. Constraints in the $M_{A}-\tan \beta$ plane from the $B \rightarrow X_{s} \gamma$ decay. The orange solid, dotted, dashed, and dash-dotted contours correspond to scenarios (a), (b), (c), and (d) as described in the text. The gray region is excluded by direct searches of MSSM Higgs bosons in the $H / A \rightarrow$ $\tau^{+} \tau^{-}$channel.
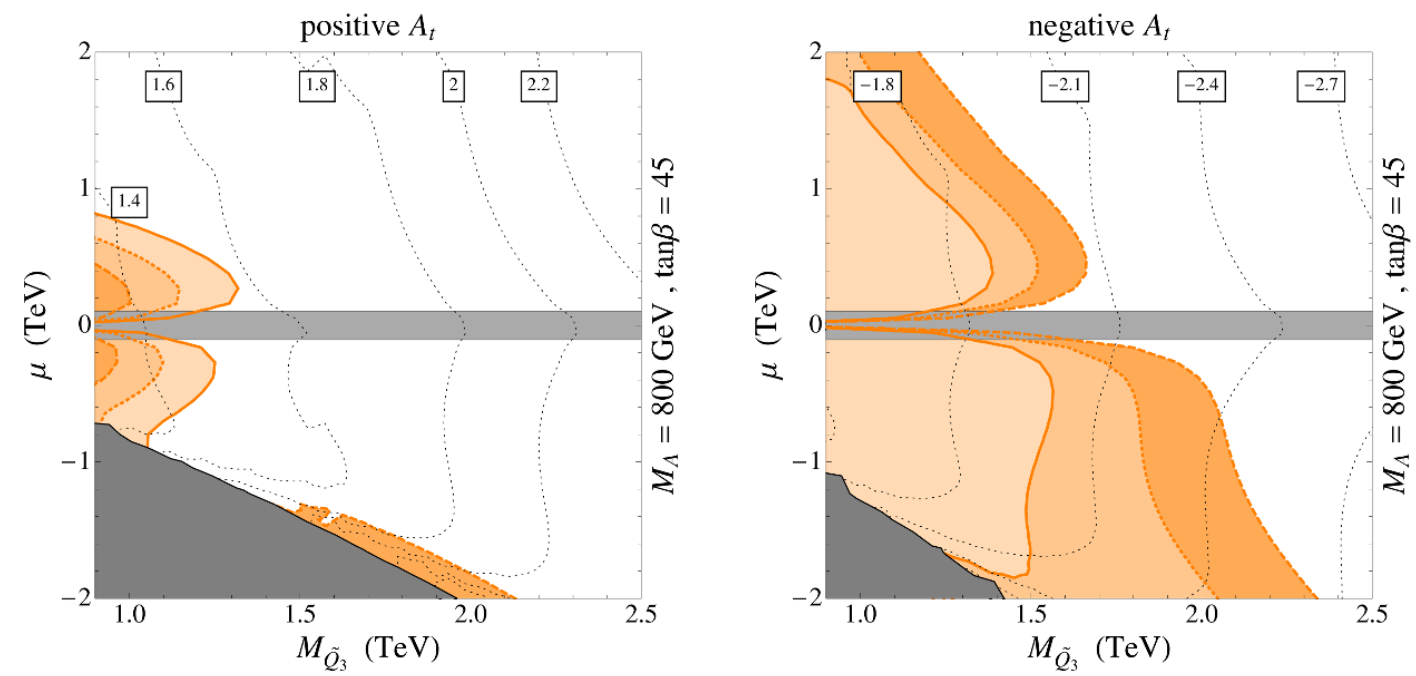

Figure 10. Constraints in the $m_{Q_{3}}-\mu$ plane from the $B \rightarrow X_{s} \gamma$ decay, for fixed $M_{3}=3 M_{2}=$ $6 M_{1}=1.5 \mathrm{TeV}$. The solid bounded regions correspond to a degenerate squark spectrum. The dashed and dotted bounded regions correspond to choosing the first two squark generations $50 \%$ heavier than the third generation squark masses, with an alignment of $\zeta=1$ and $\zeta=0.5$, respectively. The gray horizontal band corresponds to the constraint from direct searches of charginos at LEP. The vertical dotted lines show contours of constant $A_{t}$ such that $M_{h}=125 \mathrm{GeV}$. In the gray regions in the lower left corners the lightest Higgs mass is always below $M_{h}<125 \mathrm{GeV}$, taking into account a $3 \mathrm{GeV}$ theory uncertainty.

weakest in scenario (b) that has the largest $\mu=4 \mathrm{TeV}$. For the heavy squark masses chosen in figure 9, the direct searches for MSSM Higgs bosons give stronger constraints compared to $B \rightarrow X_{s} \gamma$ except for small values of $\tan \beta$. 
In the plots of figure 10, the variation of the squark masses allows the stop-chargino contribution to become important for small $m_{Q_{3}}$, demonstrating that the $\tan \beta$ vs. $M_{A}$ projection insufficiently illustrates the $B \rightarrow X_{s} \gamma$ constraint. Partial cancellations are again in effect, and we describe the relative signs of the various contributions in the following. Apart from extreme regions of parameter space, the charged Higgs contribution interferes constructively with the $\mathrm{SM}$ and enhances $\mathrm{BR}\left(B \rightarrow X_{s} \gamma\right)$. However, for the case shown, $M_{A}=800 \mathrm{GeV}$, this contribution is small. For positive (negative) $\left(\mu A_{t}\right)$, the Higgsino loop contribution come with same (opposite) sign with respect to the SM. Among the gaugino contributions, the dominant one is typically the 1-loop gluino contribution. If a splitting in the left-handed squark masses is induced radiatively, its sign depends, for positive $M_{3}$, only on the sign of $\mu$. For positive (negative) $\mu$, gluinos interfere destructively (constructively) with the SM.

The plots of figure 10 clearly show the decoupling behavior of the MSSM contributions to the $b \rightarrow s \gamma$ transition. For a degenerate squark spectrum $\left(m_{Q_{3}}=m_{Q}=m_{U_{3}}=m_{U}=\right.$ $\left.m_{D_{3}}=m_{D}=\tilde{m}\right)$ and a heavy charged Higgs, the bound from $\operatorname{BR}\left(B \rightarrow X_{s} \gamma\right)$ hardly constrains the MSSM parameter space beyond squark masses that are already excluded by direct SUSY searches, namely $\tilde{m} \gtrsim \mathcal{O}(1 \mathrm{TeV})$. In the presence of a mass splitting between the first two and the third generations of squarks, the $B \rightarrow X_{s} \gamma$ constraint can become relevant for negative $A_{t}$, since the gluino and Higgsino contributions add constructively. Squark masses significantly above $1 \mathrm{TeV}$ can be probed in that case. For positive values of $A_{t}$, on the other hand, the gluino and Higgsino loops partially cancel and the bound from $B \rightarrow X_{s} \gamma$ is barely relevant.

\subsection{Discussion of RGE effects}

Our phenomenological analysis of MSSM mass parameters serves our purpose of understanding the flavor constraints on the low energy MSSM spectrum. However, we also want to connect these constraints to parameters of a high scale SUSY parameter space. To this end, we consider a typical example in the large $\tan \beta$ and $M_{A}$ region compatible with direct $H / A \rightarrow \tau^{+} \tau^{-}$searches at the LHC. We show typical mass differences between soft parameters for squarks in the plane of the mSUGRA boundary conditions, $m_{0}$ and $m_{1 / 2}$, fixing the remaining mSUGRA parameters to $A_{0}= \pm 2 \mathrm{TeV}$ and $\tan \beta=45$. We also chose the SUSY breaking scale to be the GUT scale, $10^{16} \mathrm{GeV}$. We deviate slightly from the strict mSUGRA prescription and work in a non-universal Higgs mass (NUHM) scenario by fixing the Higgs soft mass $m_{H_{u}}^{2}=1 \mathrm{TeV}^{2}$ and adjusting $m_{H_{d}}^{2}$ at the high scale to obtain $M_{A}$ within $10 \%$ of $800 \mathrm{GeV}$ at the low scale of $Q=1 \mathrm{TeV}$. Using these boundary conditions and the usual low energy Yukawa constraints derived from fermion masses run to $Q=1 \mathrm{TeV}$, we numerically solve the RGE system dictated by 2-loop running from [194] and 1-loop radiative corrections from [195]. Our choice of $A_{0}$ typically gives the lightest SM-like Higgs a mass of $122 \pm 2 \mathrm{GeV}$. For the bulk of the region in the $\left(m_{0}, m_{1 / 2}\right)$ plane, adjusting $A_{0}$ (in particular, $A_{t}$ ) to obtain a Higgs mass of $125 \mathrm{GeV}$ changes the quantitative picture by less than a few percent. For very small $m_{0}$ and $m_{1 / 2}$, however, where some squarks or sleptons become close to tachyonic, the mass splittings can vary significantly as result of changing $A_{0}$. 
We highlight that the $B$ observable constraints can vary significantly as a result of Yukawa-induced squark mass splittings inherent in RG running, as seen in figure 6 and figure 10, respectively. In particular, the most significant mass splittings among the squarks occur as a result of the top and bottom Yukawas, where a significant enhancement of the bottom Yukawa occurs for large $\tan \beta$.

We can obtain a semi-analytic understanding of the resulting mass splittings, following the simplified 1-loop RG analysis of [196-198]. Neglecting the first- and second-generation Yukawa couplings and $\alpha_{1}^{2}$ contributions, we have

$$
\begin{aligned}
m_{Q_{3}}^{2}(t) & \approx m_{Q_{3}}^{2}(0)+I_{\alpha_{3}}+I_{\alpha_{2}}-I_{t}-I_{b} \\
m_{U_{3}}^{2}(t) & \approx m_{U_{3}}^{2}(0)+I_{\alpha_{3}}-2 I_{t} \\
m_{D_{3}}^{2}(t) & \approx m_{D_{3}}^{2}(0)+I_{\alpha_{3}}-2 I_{b}
\end{aligned}
$$

and

$$
\begin{aligned}
I_{\alpha_{3}} & \equiv \int d t\left(\frac{16}{3} \frac{\alpha_{3}}{4 \pi} M_{3}^{2}\right), \\
I_{\alpha_{2}} & \equiv \int d t\left(3 \frac{\alpha_{2}}{4 \pi} M_{2}^{2}\right), \\
I_{t} & \equiv \frac{1}{16 \pi^{2}} \int d t y_{t}^{2}\left(m_{Q}^{2}+m_{U}^{2}+m_{H_{u}}^{2}+A_{t}^{2}\right), \\
I_{b} & \equiv \frac{1}{16 \pi^{2}} \int d t y_{b}^{2}\left(m_{Q}^{2}+m_{D}^{2}+m_{H_{d}}^{2}+A_{b}^{2}\right),
\end{aligned}
$$

where $t=0$ corresponds to the GUT scale. The analogous $m_{Q_{1}}^{2}, m_{U_{1}}^{2}$, and $m_{D_{1}}^{2}$ approximations can be obtained from the above by neglecting the $I_{t}$ and $I_{b}$ contributions.

For the trilinear couplings, neglecting $\alpha_{1}$ and $A_{\tau}$,

$$
\begin{aligned}
& A_{t} \approx A_{0}+\int d t\left[\left(\frac{16}{3} \frac{\alpha_{3}}{4 \pi} M_{3}+3 \frac{\alpha_{2}}{4 \pi} M_{2}\right)-6 \frac{y_{t}^{2}}{16 \pi^{2}} A_{t}-\frac{y_{b}^{2}}{16 \pi^{2}} A_{b}\right] \\
& A_{b} \approx A_{0}+\int d t\left[\left(\frac{16}{3} \frac{\alpha_{3}}{4 \pi} M_{3}+3 \frac{\alpha_{2}}{4 \pi} M_{2}\right)-\frac{y_{t}^{2}}{16 \pi^{2}} A_{t}-6 \frac{y_{b}^{2}}{16 \pi^{2}} A_{b}\right] .
\end{aligned}
$$

The most relevant mass splittings for our analysis are

$$
\begin{aligned}
\Delta Q_{13} & \equiv \frac{m_{Q_{1}}^{2}-m_{Q_{3}}^{2}}{m_{Q_{1}}^{2}} \approx \frac{I_{t}+I_{b}}{m_{Q_{1}}^{2}} \\
\Delta U_{13} & \equiv \frac{m_{U_{1}}^{2}-m_{U_{3}}^{2}}{m_{U_{1}}^{2}} \approx \frac{2 I_{t}}{m_{U_{1}}^{2}} \\
\Delta D_{13} & \equiv \frac{m_{D_{1}}^{2}-m_{D_{3}}^{2}}{m_{D_{1}}^{2}} \approx \frac{2 I_{b}}{m_{D_{1}}^{2}} \\
\Delta Q U_{3} & \equiv \frac{m_{Q_{3}}^{2}-m_{U_{3}}^{2}}{m_{Q_{3}}^{2}} \approx \frac{I_{\alpha_{2}}+I_{t}-I_{b}}{m_{Q_{1}}^{2}-I_{t}-I_{b}} \\
\Delta Q D_{3} & \equiv \frac{m_{Q_{3}}^{2}-m_{D_{3}}^{2}}{m_{Q_{3}}^{2}} \approx \frac{I_{\alpha_{2}}-I_{t}+I_{b}}{m_{Q_{1}}^{2}-I_{t}-I_{b}} .
\end{aligned}
$$



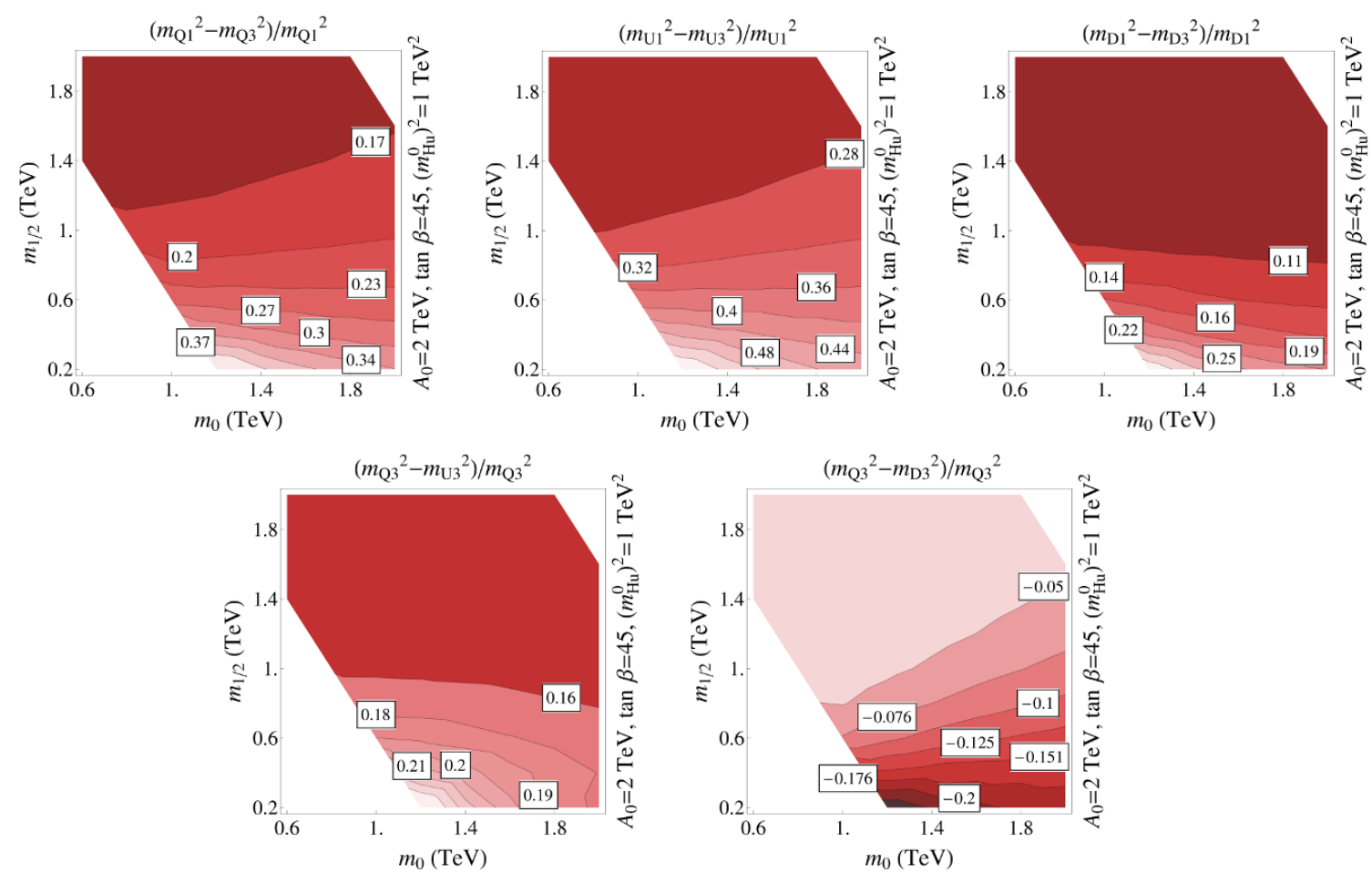

Figure 11. Contours of $\Delta Q_{13}$ (top left), $\Delta U_{13}$ (top middle), $\Delta D_{13}$ (top right), $\Delta Q U_{3}$ (bottom left), and $\Delta Q D_{3}$ (bottom right) in the $\left(m_{0}, m_{1 / 2}\right)$ plane, fixing $A_{0}=2 \mathrm{TeV},\left(m_{H_{u}}^{0}\right)^{2}=1 \mathrm{TeV}^{2}$, $\tan \beta=45$, and requiring $M_{A}=800 \mathrm{GeV}$ within $10 \%$.

From these relations we see that $\Delta U_{13}+\Delta D_{13} \approx 2 \Delta Q_{13}$, where $\Delta D_{13}$ is small compared to $\Delta U_{13}$ for small $\tan \beta$. We also expect $\Delta Q U_{3}=-\Delta Q D_{3}$ for small $m_{1 / 2}$. These relations for the various mass splittings, based on 1-loop semi-analytic results, are borne out in our numerical results, which are calculated from 2-loop RG running, and are shown in figure 11 and figure 12 .

For a gluino with mass $M_{3}=1.5 \mathrm{TeV}$ as we considered in the previous sections, we have $m_{1 / 2} \simeq 500 \mathrm{GeV}$ and therefore a significant splitting is induced among the squark masses in running down from the GUT scale. The most important splitting in the general discussion of the previous sections is $\Delta Q_{13}$, as it leads to gaugino loop contributions to FCNCs. In our mSUGRA setup, it is typically around $25 \%$ for positive $A_{0}$ and $35 \%$ for negative $A_{0}$. The splitting between the masses of the squarks decreases for larger $m_{1 / 2}$. This is due to the universal $\mathrm{SU}(3)$ contribution, $I_{\alpha_{3}}$, to $m_{Q_{3}}, m_{Q}, m_{U_{3}}, m_{U}, m_{D_{3}}$, and $m_{D}$, which dominates for large $m_{1 / 2}$.

From the approximate expressions above, we can also estimate the size of $\zeta$ resulting from running. We have

$$
\zeta \simeq \frac{I_{t}}{I_{t}+I_{b}}
$$

Even though we chose a large value of $\tan \beta=45$ for the examples shown, the bottom Yukawa effects are limited. Note that for the parameter region explored here, $\zeta \sim 80 \%$, 

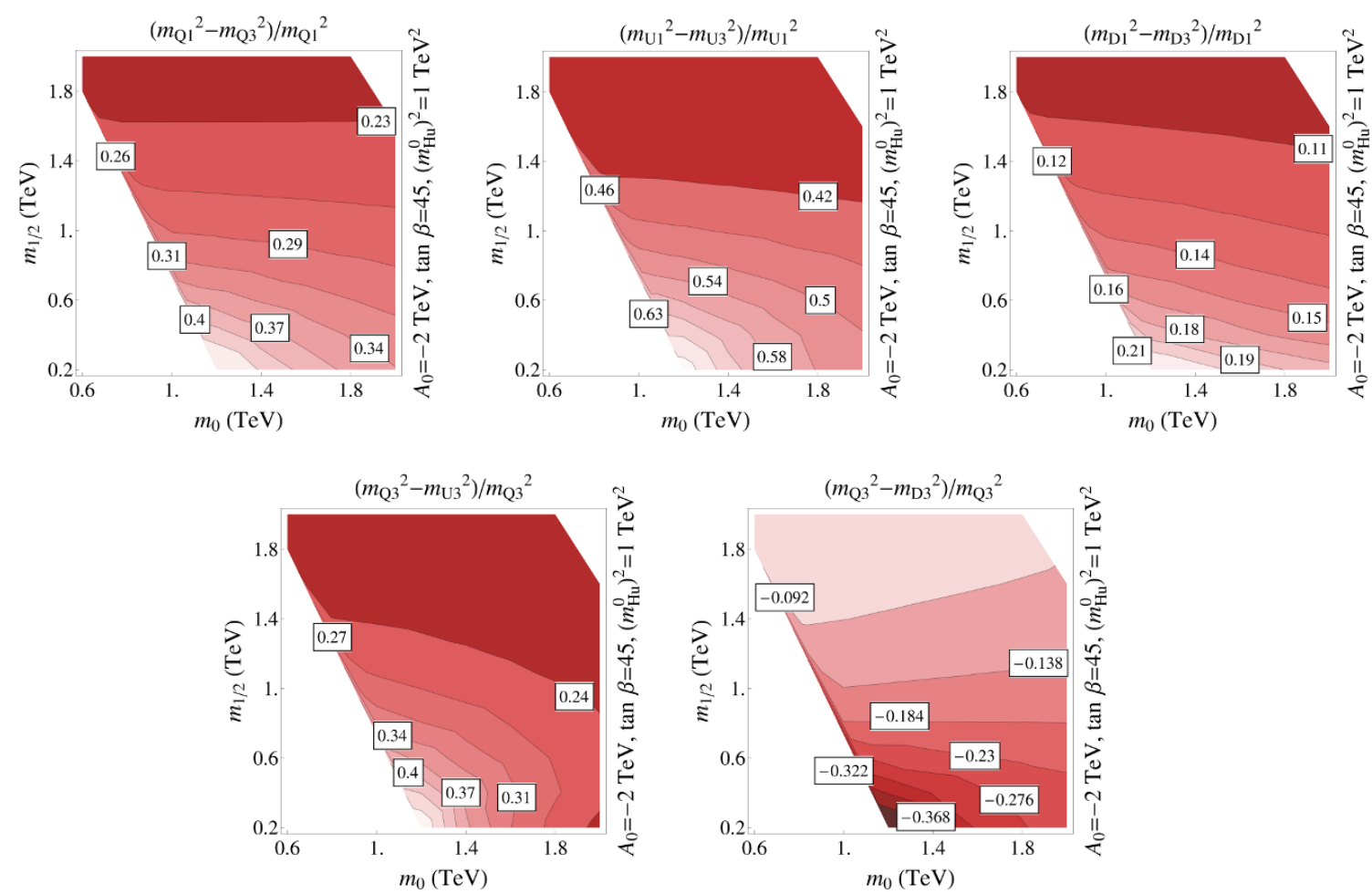

Figure 12. Same as figure 11 except $A_{0}=-2 \mathrm{TeV}$.

which means that the squark mass splitting is dominantly driven by the top Yukawa and therefore aligned in the up-sector. For smaller $\tan \beta$, the alignment parameter $\zeta$ is even closer to 1.

Note that the gaugino loop contributions to FCNCs depend approximately on the product $\zeta \times \Delta Q_{13}$. In the mSUGRA scenario discussed here, we find to a good approximation $\zeta \times \Delta Q_{13} \simeq \Delta U_{13} / 2$. In more generic setups however, this relation does not hold and we will continue to discuss the gaugino loop contributions to FCNCs in terms of $\zeta$ and $\Delta Q_{13}$ separately.

In the plots of figure 13, we show again the constraints from $B_{s} \rightarrow \mu^{+} \mu^{-}$and $B \rightarrow X_{s} \gamma$ in the $m_{Q_{3}}-\mu$ plane, this time setting the various mass splittings according to our results of the mSUGRA RGE running. In particular, we use $\Delta Q_{13}=0.35, \Delta U_{13}=0.6, \Delta D_{13}=0.15$, $\Delta Q U_{3}=0.35$, and $\Delta Q D_{3}=-0.25$, which are typical values for $m_{1 / 2} \simeq 500 \mathrm{GeV}$ and negative $A_{t}$. As we saw in the previous sections for positive $A_{t}$, the $B_{s} \rightarrow \mu^{+} \mu^{-}$constraint depends very mildly on the squark mass splitting and the $B \rightarrow X_{s} \gamma$ constraint is barely relevant. Therefore, we restrict ourselves to negative $A_{t}$. For comparison, the solid contours indicate again the constraints obtained for a degenerate squark spectrum. The dotted contours corresponds to keeping all third generation squarks degenerate and only implementing the splitting between the first two and the third generation as given by the RGE running. The dashed contours correspond to the situation where all squark mass splittings are as dictated by the RGE running. The former case behaves as expected given the analysis of 

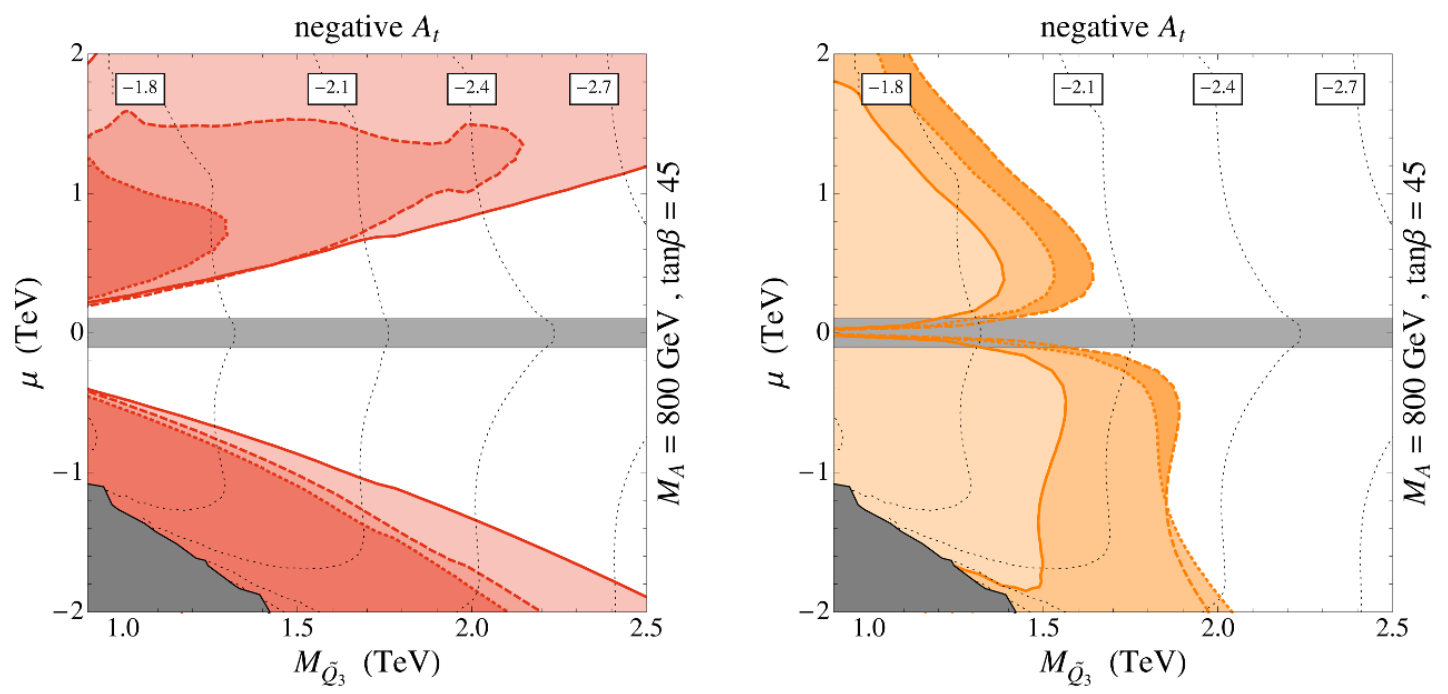

Figure 13. Constraints in the $m_{Q_{3}}-\mu$ plane from $B_{s} \rightarrow \mu^{+} \mu^{-}$(left) and $B \rightarrow X_{s} \gamma$ (right). The solid bounded regions correspond to a degenerate squark spectrum. The dashed and dotted bounded regions correspond to mass splittings in the squark spectrum implied by RGE running. In particular $\Delta Q_{13}=0.35, \Delta U_{13}=0.6, \Delta D_{13}=0.15, \Delta Q U_{3}=0.35$, and $\Delta Q D_{3}=-0.25$ for the dashed contours and $\Delta Q_{13}=0.35, \Delta U_{13}=0.6, \Delta D_{13}=0.15$, and $\Delta Q U_{3}=\Delta Q D_{3}=0$ for the dotted contours. The gray horizontal band corresponds to the constraint from direct chargino searches. The vertical dotted lines show contours of constant $A_{t}$ such that $M_{h}=125 \mathrm{GeV}$. In the gray regions in the lower left corners the lightest Higgs mass is always below $M_{h}<125 \mathrm{GeV}$, taking into account a $3 \mathrm{GeV}$ theory uncertainty.

sections 5.2 and 5.3. For the latter case, however, once mass splittings between the different types of third generation squarks are also considered, an additional effect arises. As can be seen from (5.22) and confirmed in the lower left plots of figures 11 and 12, the right handed stop is typically significantly lighter than the other third generation squarks. The light right-handed stop then increases the chargino-stop loop contributions to $B_{s} \rightarrow \mu^{+} \mu^{-}$ and to $B \rightarrow X_{s} \gamma$ leading overall to stronger constraints compared to the case of degenerate third generation squarks.

Two of the most important quantities dictated by RGEs for flavor observables are the values of $\Delta Q_{13}$ and $\zeta$. Within the assumption of flavor universality at the messanger scale, $\Delta Q_{13}$ and $\zeta$ depend mainly on the messenger scale, $\tan \beta$ and the ratio of gluino mass to squark masses. Lowering the messenger scale from the GUT scale as well as increasing the gluino mass decreases the splitting $\Delta Q_{13}$, but leaves $\zeta$ approximately invariant. Smaller (larger) values of $\tan \beta$ would decrease (increase) $\Delta Q_{13}$ and simultaneously increase (decrease) $\zeta$, leaving the product $\zeta \times \Delta Q_{13} \simeq \Delta U_{13} / 2$ approximately invariant. As we saw, making the splitting smaller strengthens the $\operatorname{BR}\left(B_{s} \rightarrow \mu^{+} \mu^{-}\right)$constraint for negative $A_{t}$, but increasing $\zeta$ will relax it. The effect of these two quantities is exactly opposite on the constraints coming from $\mathrm{BR}\left(B \rightarrow X_{s} \gamma\right)$. This complimentary behavior implies that even varying the messenger scale and $\tan \beta$, these two flavor observables will be able to constrain the parameter space efficiently. 


\section{Dark matter direct detection}

The lightest neutralino in the MSSM is an excellent thermal dark matter candidate. The lightest neutralino is a weakly interacting massive particle (WIMP) and therefore generically leads to roughly the correct order of magnitude for the observed dark matter relic density. This is particularly true in the well-tempered neutralino scenario [199], where the lightest neutralino is a mixture of the Bino and Wino or the Higgsino. In the following we do not assume any specific mechanism by which the correct dark matter relic abundance is achieved, but simply assume that the lightest neutralino in the MSSM accounts for the dark matter in the universe [200].

Neutralinos interact with SM matter and therefore dark matter direct detection limits can be used to put bounds on the MSSM parameter space, complementary to the bounds from direct searches and low energy flavor observables [59, 68, 201-209].

The Xenon100 Collaboration recently set very stringent limits on the spin-independent elastic dark matter nucleon scattering cross section [84, 211]. For dark matter masses of $\mathcal{O}(100 \mathrm{GeV})$, the bounds are as strong as $\sigma<2 \times 10^{-45} \mathrm{~cm}^{2}$, assuming canonical values for the local dark matter density, the local circular velocity and the Galactic escape velocity. Interpreted in the context of the MSSM with neutralino dark matter, these bounds are starting to probe significant parts of the parameter space.

The spin-independent elastic neutralino-proton cross-section can be written as

$$
\sigma=\frac{4 M_{\chi}^{2} m_{p}^{2}}{\pi\left(M_{\chi}+m_{p}\right)^{2}} f_{p}^{2}
$$

where $M_{\chi}$ is the mass of the lightest neutralino, $m_{p}$ is the proton mass, and

$$
\frac{f_{p}}{m_{p}}=\left(\sum_{q=u, d, s} f_{\mathrm{T}_{q}}^{p} c_{q}+\frac{2}{27} f_{\mathrm{TG}}^{p} \sum_{q=c, b, t} c_{q}\right) .
$$

The non-perturbative parameters $f_{\mathrm{T}_{q}}^{p}$ and $f_{\mathrm{TG}}^{p}=1-f_{\mathrm{T}_{u}}^{p}-f_{\mathrm{T}_{d}}^{p}-f_{\mathrm{T}_{s}}^{p}$ come from the evaluation of nuclear matrix elements. We use the latest lattice determinations in our numerical analysis [210]

$$
f_{\mathrm{T}_{u}}^{p}=f_{\mathrm{T}_{d}}^{p}=0.028, f_{\mathrm{T}_{s}}^{p}=0.0689 .
$$

These values are expected to be affected by considerable uncertainties. We assume isospin symmetry when applying the Xenon100 bounds.

For large $\tan \beta$, the dominant contributions to the coefficients, $c_{q}$, parametrizing the neutralino-quark couplings, typically come from the $t$-channel exchange of the heavy scalar $H$ and read

$$
\begin{aligned}
c_{d}^{H}=c_{s}^{H} & \simeq \frac{g_{1}^{2}}{4 M_{H}^{2}} \frac{t_{\beta}}{1+\epsilon_{s} t_{\beta}} \frac{\mu}{M_{1}^{2}-\mu^{2}}, \\
c_{b}^{H} & \simeq \frac{g_{1}^{2}}{4 M_{H}^{2}} \frac{t_{\beta}}{1+\epsilon_{b} t_{\beta}} \frac{\mu}{M_{1}^{2}-\mu^{2}} .
\end{aligned}
$$



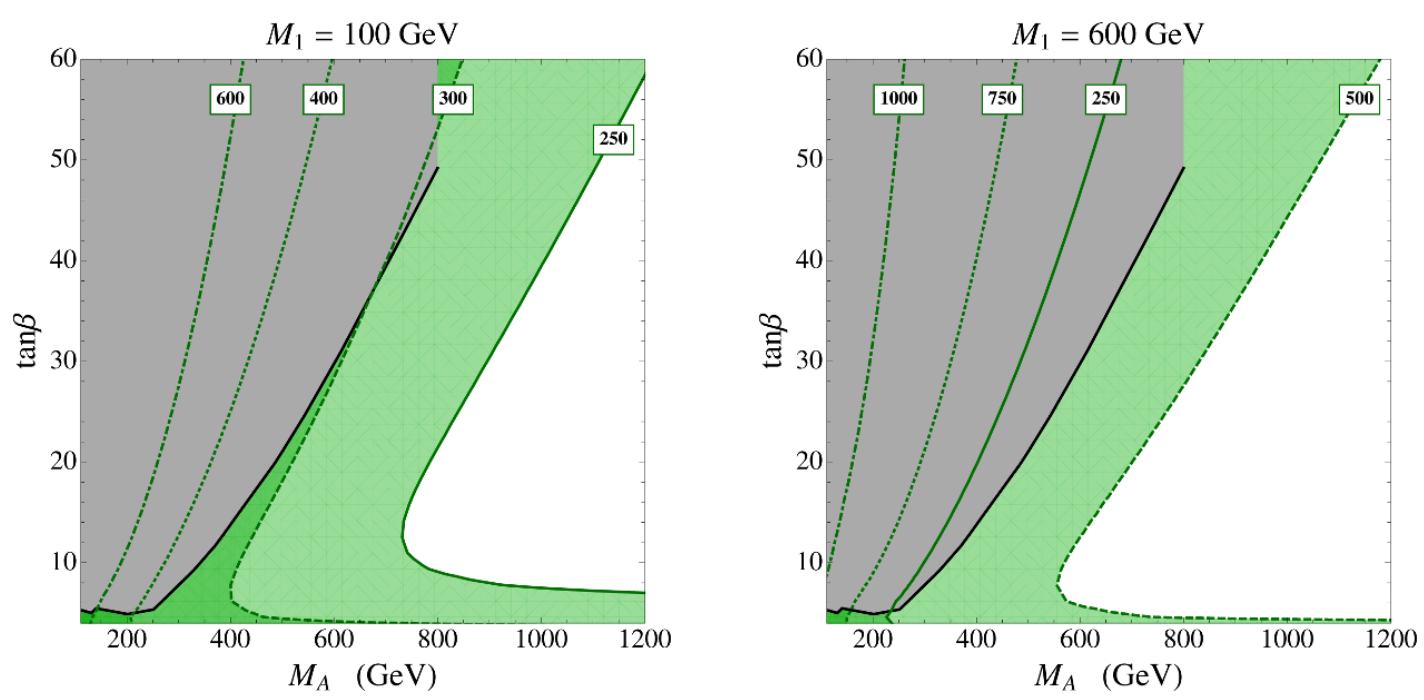

Figure 14. Constraints in the $M_{A}-\tan \beta$ plane from Dark Matter direct detection. The green solid, dashed, dotted and dash-dotted contours correspond to different values of $\mu$ as indicated. The gray region is excluded by direct searches of MSSM Higgs bosons in the $H / A \rightarrow \tau^{+} \tau^{-}$channel.

The $t$-channel exchange of the SM-like Higgs affects all $c_{q}$ approximately equally:

$$
c_{q}^{h} \simeq \frac{g_{1}^{2}}{4 M_{h}^{2}} \frac{M_{1}}{M_{1}^{2}-\mu^{2}} .
$$

While the $c_{q}^{h}$ are not enhanced by $\tan \beta$, bounds on the direct detection cross section have become so strong that the $t$-channel exchange of the SM-like Higgs is also probed.

The above expressions hold in the large $\tan \beta$ limit and assume the lightest supersymmetric particle to be mainly a bino-higgsino mixture with $M_{1} \neq \mu$. In our numerical analysis, we go beyond the large $\tan \beta$ limit: we work with neutralino mass eigenstates and include the effects from $s$-channel squark exchange, though these are always very suppressed by the squark masses.

As is evident from (6.4), (6.5), and (6.6), the neutralino-proton cross section depends strongly on $M_{1}$ and $\mu$. This can be also seen from the plots of figure 14, which show in green the regions in the canonical $M_{A}-\tan \beta$ plane that are excluded by the Xenon100 constraints. In the left plot, the bino mass is set to $M_{1}=100 \mathrm{GeV}$ with $M_{2}=2 M_{1}$ and the solid, dashed, dotted and dash-dotted contours correspond to $\mu=250 \mathrm{GeV}, 300 \mathrm{GeV}$, $400 \mathrm{GeV}$, and $600 \mathrm{GeV}$, as indicated in the plot. In the right plot, we choose a larger bino mass of $M_{1}=600 \mathrm{GeV}$, with $M_{2}=2 M_{1}$ again, and the solid, dashed, dotted and dashdotted contours correspond to $\mu=250 \mathrm{GeV}, 500 \mathrm{GeV}, 750 \mathrm{GeV}$, and $1000 \mathrm{GeV}$. Dependence on other SUSY parameters enters at the loop level through the $\epsilon_{i}$ factors in (6.4) and (6.5) and is therefore very moderate. In these plots we fix a common squarks mass, $\tilde{m}=2 \mathrm{TeV}$, a gluino mass of $M_{3}=1.5 \mathrm{TeV}$ and $A_{t}=A_{b}=A_{\tau}$ such that the lightest Higgs mass $125 \mathrm{GeV}$. 


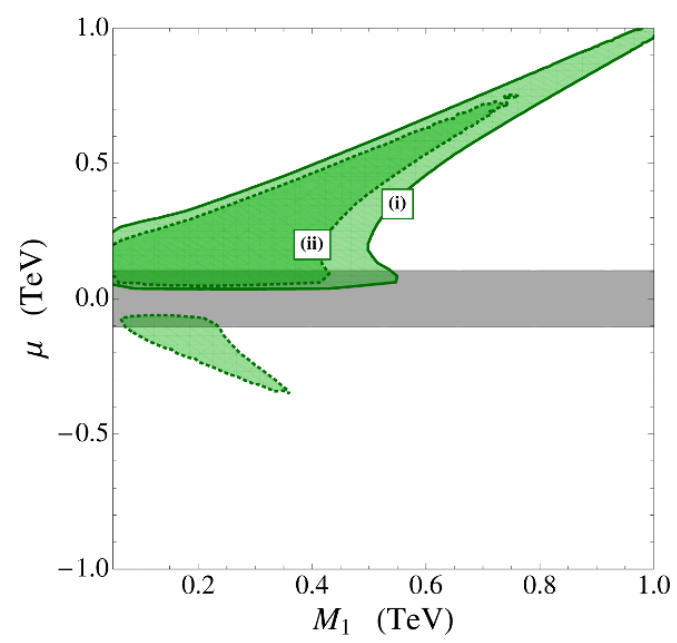

Figure 15. Constraints in the $M_{1}-\mu$ plane from Dark Matter direct detection. The solid and dashed contours correspond to different choices for $M_{A}$ and $\tan \beta$ as defined in the text. The horizontal gray band is excluded by direct chargino searches.

The strongest constraints arise if binos and higgsinos are maximally mixed, i.e. for $M_{1} \simeq \mu$. Indeed, if $M_{1}=\mu$, we find, independent of the values of $M_{A}$ and $\tan \beta$, that the exchange of the SM-like Higgs leads to direct detection cross sections that are already ruled out by the current bounds in the full range of neutralino masses up to $1 \mathrm{TeV}$. Away from bino-higgsino degeneracy, regions of parameter space open up. Still, for small heavy Higgs masses and large values of $\tan \beta$, the heavy Higgs exchange contributions can be sizable and lead to important constraints in the $M_{A}{ }^{-} \tan \beta$ plane, as long as $\mu$ and $M_{1} \lesssim 1 \mathrm{TeV}$. In the excluded regions with small $\tan \beta$ and a large heavy Higgs mass, the constraint arises from the exchange of the light Higgs.

The plot of figure 15 shows the direct detection constraints in the $M_{1}-\mu$ plane for 2 different points in the $M_{A}-\tan \beta$ plane. The solid, dashed and dotted contours correspond to $M_{A}=800 \mathrm{GeV}$ and $\tan \beta=45$ (scenario i) and $M_{A}=1 \mathrm{TeV}$ and $\tan \beta=10$ (scenario ii), both compatible with current direct searches. As already mentioned, the strongest constraints arise along the $M_{1} \simeq \mu$ line. Interestingly, the constraints for negative values of $\mu$ are considerably weaker, because for negative $\mu$, the heavy Higgs and SM like Higgs contributions interfere destructively. Observe that this behavior is opposite to that of the constraints coming from $B_{s} \rightarrow \mu^{+} \mu^{-}$and $B \rightarrow X_{s} \gamma$, which are currently weaker for positive $\mu$ (and positive $A_{t}$ ).

Note that the bounds from dark matter direct detection not only depend very strongly on various MSSM parameters, but are also affected by various uncertainties, e.g. from the nuclear matrix elements, and astrophysical uncertainties, in particular the dark matter velocity distribution. Moreover, they also depend crucially on the assumption that the dark matter of the universe indeed consist entirely of MSSM neutralinos. If neutralinos only make up a (small) fraction of the dark matter, the bounds can be relaxed considerably and even avoided completely. 


\section{Conclusions}

In this work, we evaluated the status of the minimal supersymmetric standard model with minimal flavor violation in light of the recent Higgs discovery as well as constraints from collider searches, flavor measurements, and dark matter direct detection experiments. In concert, these complementary probes provide valuable constraints on the MSSM parameter space. In particular, we showed that flavor bounds can be stronger than bounds from direct searches for heavy MSSM Higgs particles or supersymmetric particles, even in the restrictive framework of MFV.

Throughout our analysis, we consistently implemented the most general structure of the soft SUSY breaking terms compatible with the MFV ansatz, i.e. allowing splitting between the first two and the third generations of squarks. We demonstrated that, in addition to the typical pMSSM parameters, an additional parameter, $\zeta$, reflective of the alignment of the mass splitting of the left-handed squarks, is required to discuss the flavor phenomenology of this framework. In the presence of such splitting, this parameter controls the size of gaugino-squark loop contributions to FCNCs. Possible cancellations between gaugino and higgsino loop contributions have a very strong dependence on $\zeta$. We showed its impact in the $B_{s} \rightarrow \mu^{+} \mu^{-}$and $B \rightarrow X_{s} \gamma$ decays and presented expectations for its magnitude as dictated by RGE running.

We discussed the constraints from direct searches of the heavy MSSM Higgs bosons. Bounds from $H / A \rightarrow \tau^{+} \tau^{-}$searches mainly depend on $M_{A}$ and $\tan \beta$ and are robust against variations of other SUSY parameters. Separately, searches in the $H / A \rightarrow b b$ channel show a stronger dependence on the parameters under consideration, in particular on the sign and magnitude of the Higgsino mass parameter, $\mu$, and therefore provide complementary information. Currently, however, the $H / A \rightarrow \tau^{+} \tau^{-}$searches are more strongly constraining for the considered scenarios.

On the flavor side, we considered the tree level decay $B \rightarrow \tau \nu$ as well as the loop induced FCNC processes $B_{s} \rightarrow \mu^{+} \mu^{-}$and $B \rightarrow X_{s} \gamma$. The recent experimental updates on the $\mathrm{BR}(B \rightarrow \tau \nu)$ show reasonable agreement with the SM prediction. At tree level, charged Higgs contributions to $B \rightarrow \tau \nu$ interfere destructively with the SM amplitude. At the loop level, a net constructive interference is in principle possible for very large and negative $\mu \tan \beta$. However, we find that the corresponding regions of parameter space are excluded by vacuum meta-stability considerations. The $B \rightarrow \tau \nu$ decay can lead to constraints in the $M_{A}-\tan \beta$ plane also for $M_{A}>800 \mathrm{GeV}$ where current direct searches for MSSM Higgs bosons end. For such heavy Higgs bosons however, $B \rightarrow \tau \nu$ only probes very large values of $\tan \beta \gtrsim 60$. The $B \rightarrow \tau \nu$ constraints depend only moderately on SUSY parameters other than $M_{A}$ and $\tan \beta$. In particular, they depend only weakly on possible new sources of flavor violation beyond the MFV ansatz.

The constraints from the FCNC decays on the $\tan \beta-M_{A}$ plane depend crucially on several parameters, in particular the Higgsino mass, $\mu$, the stop trilinear coupling, $A_{t}$, the gluino mass, $M_{3}$, the mass splitting of the left-handed squarks, $\Delta Q_{13}$, and its alignment in flavor space, $\zeta$. The current experimental bounds on the $\operatorname{BR}\left(B_{s} \rightarrow \mu^{+} \mu^{-}\right)$lead to strong constraints in the large $\tan \beta$ regime of the MSSM with MFV. Constraints are particularly 
strong if the MSSM contributions interfere constructively with the SM, which happens for $\operatorname{sign}\left(\mu A_{t}\right)=-1$. In that case, even for moderately large $\tan \beta \sim 30$, heavy Higgs masses of up to $1 \mathrm{TeV}$ can be probed. Note that these bounds can have a strong dependance on $\Delta Q_{13}$ and $\zeta$. For negative $A_{t}$, they become less constraining for larger values of $\zeta$ and larger $\Delta Q_{13}$. The main dependence is to a good approximation on the product $\zeta \times \Delta Q_{13}$. In a mSUGRA setup this product is correlated with the mass splitting of the right-handed up squarks $\zeta \times \Delta Q_{13} \simeq \Delta U_{13} / 2$. From our RGE analysis of a simple mSUGRA model, we expect $\zeta=0.8$ for $\tan \beta=45$ and $\zeta$ even closer to 1 for smaller $\tan \beta$. We also find $\Delta Q_{13} \sim 20 \%$ to $35 \%$, which should be approximately generic for SUSY breaking models with flavor universal soft masses at the GUT scale and light gluinos $M_{3} \lesssim 2 \mathrm{TeV}$. Such values have visible impact on the bounds derived from $\operatorname{BR}\left(B_{s} \rightarrow \mu^{+} \mu^{-}\right)$. If a lower bound on $\operatorname{BR}\left(B_{s} \rightarrow \mu^{+} \mu^{-}\right)$above one half of the SM prediction is established in the future, destructively interfering SUSY contributions will also be highly constrained.

It is important to stress that the MSSM contributions to $B_{s} \rightarrow \mu^{+} \mu^{-}$do not necessarily decouple with the SUSY scale, but can probe masses of SUSY particles far above the scales that are currently reached by direct searches. On the other hand, the MSSM contributions to the $B \rightarrow X_{s} \gamma$ decay do decouple with the SUSY scale, but even so, the $B \rightarrow X_{s} \gamma$ decay can give nontrivial constraints on the MFV MSSM parameter space. If SUSY particles are heavier than $\sim 2 \mathrm{TeV}$, charged Higgs contributions to $\mathrm{BR}\left(B \rightarrow X_{s} \gamma\right)$ still lead to a constraint for small $M_{A}$ which is almost independent of all other parameters if $\tan \beta$ is not large. The corresponding bound in the $M_{A}-\tan \beta$ plane can be stronger than the bounds from direct searches for $\tan \beta \lesssim 5$ and rules out $M_{A} \lesssim 300 \mathrm{GeV}$ if squarks are decoupled. For a TeV scale SUSY spectrum, SUSY loops can also contribute sizably to $B \rightarrow X_{s} \gamma$. This is particularly true for a sizable mass splitting $\Delta Q_{13}$ and negative values of $A_{t}$, where Higgsino and gluino loop contributions add up constructively. Again, $\zeta$ can impact the implied constraints significantly. In contrast to $B_{s} \rightarrow \mu^{+} \mu^{-}$, however, the bounds become stronger for larger values of $\zeta$, if $A_{t}$ is negative. A main conclusion of our work is that the current bounds from $B \rightarrow X_{s} \gamma$ and $B_{s} \rightarrow \mu^{+} \mu^{-}$are minimized if both $\mu$ and $A_{t}$ are positive. In this region of parameter space, $(g-2)_{\mu}$ generically prefers a positive sign of $M_{2}$.

We remark that the discussed FCNC $B$ decays are also sensitive to sources of flavor violation beyond MFV. For the MSSM with generic flavor violating structures, however, bounds from FCNC processes become significantly more model dependent.

Finally, we analyzed the impact of the updated bounds from dark matter direct detection searches. We found that the parameter space region where $M_{1} \simeq \mu$ is ruled out throughout the whole $M_{A}$-tan $\beta$ plane. Away from bino-higgsino degeneracy, the current Xenon100 bounds still give strong constraints in the $M_{A}$-tan $\beta$ plane as long as $M_{1}$ and $\mu$ are below $1 \mathrm{TeV}$ and $\mu$ is positive. The direct detection bounds are minimized for negative $\mu$, where light and heavy scalar contributions to the neutralino-proton cross section partially cancel. These direct detection constraints are the least robust among the considered bounds, since they are subject to important nuclear and astrophysical uncertainties and depend crucially on the assumption that the lightest MSSM neutralino constitutes the entire dark matter in the universe.

In summary, we presented the viable MSSM parameter space using the MFV assump- 
tion, incorporating the discovery of a Higgs state at $125 \mathrm{GeV}$, the null direct search results for supersymmetric particles and for $H / A \rightarrow \tau^{+} \tau^{-}$and $b b$, and constraints from $B$ and $K$ observables as well as dark matter direct detection searches. We also discussed and imposed electroweak vacuum meta-stability requirements, and we illustrated expectations for $B$ flavor bounds arising from a renormalization group running analysis of generic minimal supergravity models. Throughout, we have emphasized the connection between flavor observables and direct collider searches in exploring the MSSM parameter space. This complementarity is not only important for understanding the present status of the MSSM with MFV, but it is also central to interpreting future experimental discoveries.

\section{Acknowledgments}

We would like to acknowledge helpful discussions with Stefania Gori, Arjun Menon and Carlos Wagner. We thank the Aspen Center for Physics for warm hospitality where part of this work was completed. The Aspen Center for Physics is supported by the National Science Foundation Grant No. PHY-1066293. W.A. thanks the Galileo Galilei Institute for Theoretical Physics for warm hospitality and the INFN for partial support during the completion of this work. Fermilab is operated by Fermi Research Alliance, LLC under Contract No. De-AC02-07CH11359 with the United States Department of Energy. N.R.S is supported by the DoE grant No. DE-SC0007859.

\section{A Loop functions}

The loop induced "wrong" Higgs couplings involve a single loop function

$$
g(x, y, z)=\frac{x \log x}{(x-y)(x-z)}+\frac{y \log y}{(y-x)(y-z)}+\frac{z \log z}{(z-x)(z-y)} .
$$

The loop functions $h_{7,8}$ enter the charged Higgs contributions to the $b \rightarrow s \gamma$ transition

$$
\begin{aligned}
& h_{7}(x)=\frac{3-5 x}{12(1-x)^{2}}+\frac{2-3 x}{6(1-x)^{3}} \log x, \\
& h_{8}(x)=\frac{3-x}{4(1-x)^{2}}+\frac{1}{2(1-x)^{3}} \log x .
\end{aligned}
$$

The loop functions that enter the Higgsino, gluino, and Wino contributions to the $b \rightarrow s \gamma$ transition can be written as

$$
\begin{aligned}
& f_{7}^{\tilde{H}}=f_{1}+\frac{2}{3} f_{2}, \quad f_{7}^{\tilde{g}}=-\frac{8}{9} f_{2}, \quad f_{7}^{\tilde{W}}=-f_{3}-\frac{1}{2} f_{2}, \\
& f_{8}^{\tilde{H}}=f_{2}, \quad f_{8}^{\tilde{g}}=-\frac{1}{3} f_{2}-3 f_{1}, \quad f_{8}^{\tilde{W}}=-\frac{3}{2} f_{2},
\end{aligned}
$$


with

$$
\begin{aligned}
f_{1}(x, y, z)= & -\frac{x^{2} \log x}{(x-y)(x-z)^{3}}-\frac{y^{2} \log y}{(y-x)(y-z)^{3}} \\
& -\frac{\left(x^{2} y^{2}-3 x y z^{2}+(x+y) z^{3}\right) \log z}{(x-z)^{3}(y-z)^{3}}+\frac{x(z-3 y)+z(y+z)}{2(x-z)^{2}(y-z)^{2}}, \\
f_{2}(x, y, z)= & \frac{x z \log x}{(x-y)(x-z)^{3}}+\frac{y z \log y}{(y-x)(y-z)^{3}} \\
& +\frac{z\left(x y(x+y)-3 x y z+z^{3}\right) \log z}{(x-z)^{3}(y-z)^{3}}+\frac{z(y-3 z)+x(y+z)}{2(x-z)^{2}(y-z)^{2}}, \\
f_{3}(x, y, z)= & -\frac{z^{2} \log x}{(x-y)(x-z)^{3}}-\frac{z^{2} \log y}{(y-x)(y-z)^{3}} \\
& -\frac{z^{2}\left(x^{2}+x y+y^{2}-3(x+y) z+3 z^{2}\right) \log z}{(x-z)^{3}(y-z)^{3}}+\frac{x(y-3 z)+z(5 z-3 y)}{2(x-z)^{2}(y-z)^{2}} .
\end{aligned}
$$

Open Access. This article is distributed under the terms of the Creative Commons Attribution License which permits any use, distribution and reproduction in any medium, provided the original author(s) and source are credited.

\section{References}

[1] ATLAS collaboration, Search for pair-produced massive coloured scalars in four-jet final states with the ATLAS detector in proton-proton collisions at $\sqrt{s}=7 \mathrm{TeV}$, arXiv:1210.4826 [INSPIRE].

[2] ATLAS collaboration, Search for pair production of massive particles decaying into three quarks with the ATLAS detector in $\sqrt{s}=7 \mathrm{TeV}$ pp collisions at the LHC, JHEP 12 (2012) 086 [arXiv: 1210.4813] [INSPIRE].

[3] ATLAS collaboration, Search for R-parity-violating supersymmetry in events with four or more leptons in $\sqrt{s}=7 \mathrm{TeV}$ pp collisions with the ATLAS detector, JHEP 12 (2012) 124 [arXiv: 1210.4457] [INSPIRE].

[4] ATLAS collaboration, Search for dark matter candidates and large extra dimensions in events with a jet and missing transverse momentum with the ATLAS detector, arXiv:1210.4491 [INSPIRE].

[5] ATLAS collaboration, Search for direct chargino production in anomaly-mediated supersymmetry breaking models based on a disappearing-track signature in pp collisions at $\sqrt{s}=7 \mathrm{TeV}$ with the ATLAS detector, JHEP 01 (2013) 131 [arXiv:1210.2852] [INSPIRE].

[6] ATLAS collaboration, Search for Supersymmetry in Events with Large Missing Transverse Momentum, Jets and at Least One Tau Lepton in $7 \mathrm{TeV}$ Proton-Proton Collision Data with the ATLAS Detector, Eur. Phys. J. C 72 (2012) 2215 [arXiv:1210.1314] [InSPIRE].

[7] ATLAS collaboration, Search for dark matter candidates and large extra dimensions in events with a photon and missing transverse momentum in pp collision data at $\sqrt{s}=7 \mathrm{TeV}$ with the ATLAS detector, arXiv:1209.4625 [INSPIRE]. 
[8] ATLAS collaboration, Search for a heavy top-quark partner in final states with two leptons with the ATLAS detector at the LHC, JHEP 11 (2012) 094 [arXiv:1209.4186] [INSPIRE].

[9] ATLAS collaboration, Search for light top squark pair production in final states with leptons and $b^{-}$jets with the ATLAS detector in $\sqrt{s}=7 \mathrm{TeV}$ proton-proton collisions, arXiv:1209.2102 [INSPIRE].

[10] ATLAS collaboration, Search for diphoton events with large missing transverse momentum in $7 \mathrm{TeV}$ proton-proton collision data with the ATLAS detector, Phys. Lett. B 718 (2012) 411 [arXiv: 1209.0753] [INSPIRE].

[11] ATLAS collaboration, Further search for supersymmetry at $\sqrt{s}=7 \mathrm{TeV}$ in final states with jets, missing transverse momentum and isolated leptons with the ATLAS detector, Phys. Rev. D 86 (2012) 092002 [arXiv:1208.4688] [INSPIRE].

[12] ATLAS collaboration, Search for light scalar top quark pair production in final states with two leptons with the ATLAS detector in $\sqrt{s}=7$ TeV proton-proton collisions, Eur. Phys. J. C 72 (2012) 2237 [arXiv: 1208.4305] [InSPIRE].

[13] ATLAS collaboration, Search for direct production of charginos and neutralinos in events with three leptons and missing transverse momentum in $\sqrt{s}=7 \mathrm{TeV} p p$ collisions with the ATLAS detector, Phys. Lett. B 718 (2013) 841 [arXiv:1208.3144] [InSPIRE].

[14] ATLAS collaboration, Search for direct slepton and gaugino production in final states with two leptons and missing transverse momentum with the ATLAS detector in pp collisions at $\sqrt{s}=7$ TeV, Phys. Lett. B 718 (2013) 879 [arXiv:1208.2884] [INSPIRE].

[15] ATLAS collaboration, Search for direct top squark pair production in final states with one isolated lepton, jets and missing transverse momentum in $\sqrt{s}=7 \mathrm{TeV}$ pp collisions using 4.7 fb $\mathrm{fb}^{-1}$ of ATLAS data, Phys. Rev. Lett. 109 (2012) 211803 [arXiv:1208.2590] [INSPIRE].

[16] ATLAS collaboration, Search for a supersymmetric partner to the top quark in final states with jets and missing transverse momentum at $\sqrt{s}=7 \mathrm{TeV}$ with the ATLAS detector, arXiv: 1208.1447 [INSPIRE].

[17] ATLAS collaboration, Search for squarks and gluinos with the ATLAS detector in final states with jets and missing transverse momentum using $4.7 \mathrm{fb}^{-1}$ of $\sqrt{s}=7 \mathrm{TeV}$ proton-proton collision data, arXiv:1208.0949 [INSPIRE].

[18] ATLAS collaboration, Search for top and bottom squarks from gluino pair production in final states with missing transverse energy and at least three b-jets with the ATLAS detector, Eur. Phys. J. C 72 (2012) 2174 [arXiv: 1207.4686] [INSPIRE].

[19] ATLAS collaboration, Hunt for new phenomena using large jet multiplicities and missing transverse momentum with ATLAS in $4.7 \mathrm{fb}^{-1}$ of $\sqrt{s}=7 \mathrm{TeV}$ proton-proton collisions, JHEP 07 (2012) 167 [arXiv:1206.1760] [INSPIRE].

[20] CMS collaboration, Search for physics beyond the standard model in events with a $Z$ boson, jets and missing transverse energy in pp collisions at $\sqrt{s}=7 \mathrm{TeV}$, Phys. Lett. B 716 (2012) 260 [arXiv: 1204.3774] [INSPIRE].

[21] CMS collaboration, Search for anomalous production of multilepton events in pp collisions at $\sqrt{s}=7 \mathrm{TeV}$, JHEP 06 (2012) 169 [arXiv:1204.5341] [INSPIRE].

[22] CMS collaboration, Search for new physics in events with same-sign dileptons and b-tagged jets in pp collisions at $\sqrt{s}=7 \mathrm{TeV}$, JHEP 08 (2012) 110 [arXiv:1205.3933] [INSPIRE]. 
[23] CMS collaboration, Search for new physics with same-sign isolated dilepton events with jets and missing transverse energy, Phys. Rev. Lett. 109 (2012) 071803 [arXiv:1205.6615] [INSPIRE].

[24] CMS collaboration, Search for new physics in events with opposite-sign leptons, jets and missing transverse energy in pp collisions at $\sqrt{s}=7$ TeV, Phys. Lett. B 718 (2013) 815 [arXiv: 1206.3949] [INSPIRE].

[25] CMS collaboration, Search for new physics in the multijet and missing transverse momentum final state in proton-proton collisions at $\sqrt{s}=7$ TeV, Phys. Rev. Lett. 109 (2012) 171803 [arXiv: 1207.1898] [INSPIRE].

[26] CMS collaboration, Search for supersymmetry in hadronic final states using MT2 in pp collisions at $\sqrt{s}=7 \mathrm{TeV}$, JHEP 10 (2012) 018 [arXiv:1207.1798] [INSPIRE].

[27] CMS collaboration, Search for supersymmetry in events with b-quark jets and missing transverse energy in pp collisions at 7 TeV, Phys. Rev. D 86 (2012) 072010 [arXiv:1208.4859] [INSPIRE].

[28] CMS collaboration, Search for electroweak production of charginos and neutralinos using leptonic final states in pp collisions at $\sqrt{s}=7$ TeV, JHEP 11 (2012) 147 [arXiv:1209.6620] [INSPIRE].

[29] CMS collaboration, Search for supersymmetry in events with photons and low missing transverse energy in pp collisions at $\sqrt{s}=7$ TeV, Phys. Lett. B. (2012) [arXiv:1210.2052] [INSPIRE].

[30] ATLAS collaboration, Observation of a new particle in the search for the Standard Model Higgs boson with the ATLAS detector at the LHC, Phys. Lett. B 716 (2012) 1 [arXiv: 1207.7214] [INSPIRE].

[31] CMS collaboration, Observation of a new boson at a mass of $125 \mathrm{GeV}$ with the CMS experiment at the LHC, Phys. Lett. B 716 (2012) 30 [arXiv:1207.7235] [InSPIRE].

[32] L.J. Hall, D. Pinner and J.T. Ruderman, A Natural SUSY Higgs Near 126 GeV, JHEP 04 (2012) 131 [arXiv:1112.2703] [INSPIRE].

[33] H. Baer, V. Barger and A. Mustafayev, Implications of a 125 GeV Higgs scalar for LHC SUSY and neutralino dark matter searches, Phys. Rev. D 85 (2012) 075010 [arXiv:1112.3017] [INSPIRE].

[34] J.L. Feng, K.T. Matchev and D. Sanford, Focus Point Supersymmetry Redux, Phys. Rev. D 85 (2012) 075007 [arXiv: 1112.3021] [INSPIRE].

[35] S. Heinemeyer, O. Stal and G. Weiglein, Interpreting the LHC Higgs Search Results in the MSSM, Phys. Lett. B 710 (2012) 201 [arXiv:1112.3026] [InSPIRE].

[36] A. Arbey, M. Battaglia, A. Djouadi, F. Mahmoudi and J. Quevillon, Implications of a 125 GeV Higgs for supersymmetric models, Phys. Lett. B 708 (2012) 162 [arXiv:1112.3028] [INSPIRE].

[37] A. Arbey, M. Battaglia and F. Mahmoudi, Constraints on the MSSM from the Higgs Sector: A pMSSM Study of Higgs Searches, $B_{s}^{0} \rightarrow \mu^{+} \mu^{-}$and Dark Matter Direct Detection, Eur. Phys. J. C 72 (2012) 1906 [arXiv:1112.3032] [InSPIRE].

[38] P. Draper, P. Meade, M. Reece and D. Shih, Implications of a 125 GeV Higgs for the MSSM and Low-Scale SUSY Breaking, Phys. Rev. D 85 (2012) 095007 [arXiv:1112.3068] [INSPIRE]. 
[39] M. Carena, S. Gori, N.R. Shah and C.E. Wagner, A $125 \mathrm{GeV}$ SM-like Higgs in the MSSM and the $\gamma \gamma$ rate, JHEP 03 (2012) 014 [arXiv: 1112.3336] [INSPIRE].

[40] U. Ellwanger, A Higgs boson near $125 \mathrm{GeV}$ with enhanced di-photon signal in the NMSSM, JHEP 03 (2012) 044 [arXiv: 1112.3548] [INSPIRE].

[41] O. Buchmueller et al., Higgs and Supersymmetry, Eur. Phys. J. C 72 (2012) 2020 [arXiv: 1112.3564] [INSPIRE].

[42] S. Akula, B. Altunkaynak, D. Feldman, P. Nath and G. Peim, Higgs Boson Mass Predictions in SUGRA Unification, Recent LHC-7 Results and Dark Matter, Phys. Rev. D 85 (2012) 075001 [arXiv: 1112.3645] [INSPIRE].

[43] M. Kadastik, K. Kannike, A. Racioppi and M. Raidal, Implications of the $125 \mathrm{GeV}$ Higgs boson for scalar dark matter and for the CMSSM phenomenology, JHEP 05 (2012) 061 [arXiv: 1112.3647] [INSPIRE].

[44] J. Cao, Z. Heng, D. Li and J.M. Yang, Current experimental constraints on the lightest Higgs boson mass in the constrained MSSM, Phys. Lett. B 710 (2012) 665 [arXiv:1112.4391] [INSPIRE].

[45] J.F. Gunion, Y. Jiang and S. Kraml, The Constrained NMSSM and Higgs near $125 \mathrm{GeV}$, Phys. Lett. B 710 (2012) 454 [arXiv: 1201.0982] [INSPIRE].

[46] S. King, M. Muhlleitner and R. Nevzorov, NMSSM Higgs Benchmarks Near 125 GeV, Nucl. Phys. B 860 (2012) 207 [arXiv: 1201.2671] [INSPIRE].

[47] Z. Kang, J. Li and T. Li, On Naturalness of the MSSM and NMSSM, JHEP 11 (2012) 024 [arXiv:1201.5305] [INSPIRE].

[48] L. Aparicio, D. Cerdeno and L. Ibáñez, A 119-125 GeV Higgs from a string derived slice of the CMSSM, JHEP 04 (2012) 126 [arXiv:1202.0822] [INSPIRE].

[49] J. Ellis and K.A. Olive, Revisiting the Higgs Mass and Dark Matter in the CMSSM, Eur. Phys. J. C 72 (2012) 2005 [arXiv:1202.3262] [INSPIRE].

[50] H. Baer, V. Barger and A. Mustafayev, Neutralino dark matter in mSUGRA/CMSSM with a 125 GeV light Higgs scalar, JHEP 05 (2012) 091 [arXiv: 1202.4038] [INSPIRE].

[51] J.-J. Cao, Z.-X. Heng, J.M. Yang, Y.-M. Zhang and J.-Y. Zhu, A SM-like Higgs near $125 \mathrm{GeV}$ in low energy SUSY: a comparative study for MSSM and NMSSM, JHEP 03 (2012) 086 [arXiv: 1202.5821] [INSPIRE].

[52] L. Maiani, A. Polosa and V. Riquer, Probing Minimal Supersymmetry at the LHC with the Higgs Boson Masses, New J. Phys. 14 (2012) 073029 [arXiv: 1202.5998] [inSPIRE].

[53] N.D. Christensen, T. Han and S. Su, MSSM Higgs Bosons at The LHC, Phys. Rev. D 85 (2012) 115018 [arXiv: 1203.3207] [INSPIRE].

[54] D.A. Vasquez et al., The $125 \mathrm{GeV}$ Higgs in the NMSSM in light of LHC results and astrophysics constraints, Phys. Rev. D 86 (2012) 035023 [arXiv:1203.3446] [INSPIRE].

[55] M.A. Ajaib, I. Gogoladze, F. Nasir and Q. Shafi, Revisiting mGMSB in Light of a $125 \mathrm{GeV}$ Higgs, Phys. Lett. B 713 (2012) 462 [arXiv:1204.2856] [inSPIRE].

[56] F. Brummer, S. Kraml and S. Kulkarni, Anatomy of maximal stop mixing in the MSSM, JHEP 08 (2012) 089 [arXiv: 1204.5977] [INSPIRE].

[57] J.L. Feng and D. Sanford, A Natural 125 GeV Higgs Boson in the MSSM from Focus Point Supersymmetry with A-Terms, Phys. Rev. D 86 (2012) 055015 [arXiv:1205.2372] [INSPIRE]. 
[58] M. Carena, S. Gori, N.R. Shah, C.E. Wagner and L.-T. Wang, Light Stau Phenomenology and the Higgs $\gamma \gamma$ Rate, JHEP 07 (2012) 175 [arXiv: 1205.5842] [INSPIRE].

[59] A. Fowlie et al., The CMSSM Favoring New Territories: The Impact of New LHC Limits and a 125 GeV Higgs, Phys. Rev. D 86 (2012) 075010 [arXiv:1206.0264] [INSPIRE].

[60] K. Blum, R.T. D'Agnolo and J. Fan, Natural SUSY Predicts: Higgs Couplings, JHEP 01 (2013) 057 [arXiv: 1206.5303] [INSPIRE].

[61] M.W. Cahill-Rowley, J.L. Hewett, A. Ismail and T.G. Rizzo, The Higgs Sector and Fine-Tuning in the pMSSM, Phys. Rev. D 86 (2012) 075015 [arXiv:1206.5800] [INSPIRE].

[62] R. Benbrik et al., Confronting the MSSM and the NMSSM with the Discovery of a Signal in the two Photon Channel at the LHC, Eur. Phys. J. C 72 (2012) 2171 [arXiv:1207.1096] [INSPIRE].

[63] A. Arbey, M. Battaglia, A. Djouadi and F. Mahmoudi, The Higgs sector of the phenomenological MSSM in the light of the Higgs boson discovery, JHEP 09 (2012) 107 [arXiv: 1207.1348] [INSPIRE].

[64] S. Akula, P. Nath and G. Peim, Implications of the Higgs Boson Discovery for mSUGRA, Phys. Lett. B 717 (2012) 188 [arXiv:1207.1839] [InSPIRE].

[65] H. An, T. Liu and L.-T. Wang, 125 GeV Higgs Boson, Enhanced Di-photon Rate and Gauged U(1) $P Q$-extended MSSM, Phys. Rev. D 86 (2012) 075030 [arXiv:1207.2473] [INSPIRE].

[66] J. Cao, Z. Heng, J.M. Yang and J. Zhu, Status of low energy SUSY models confronted with the LHC 125 GeV Higgs data, JHEP 10 (2012) 079 [arXiv: 1207.3698] [INSPIRE].

[67] G.F. Giudice, P. Paradisi, A. Strumia and A. Strumia, Correlation between the Higgs Decay Rate to Two Photons and the Muon g-2, JHEP 10 (2012) 186 [arXiv:1207.6393] [INSPIRE].

[68] O. Buchmueller et al., The CMSSM and NUHM1 in Light of $7 \mathrm{TeV} L H C, B_{s}$ to $\mu^{+} \mu^{-}$and XENON100 Data, Eur. Phys. J. C 72 (2012) 2243 [arXiv:1207.7315] [INSPIRE].

[69] J.R. Espinosa, C. Grojean, V. Sanz and M. Trott, NSUSY fits, JHEP 12 (2012) 077 [arXiv: 1207.7355] [INSPIRE].

[70] K. Schmidt-Hoberg and F. Staub, Enhanced $h \rightarrow \gamma \gamma$ rate in MSSM singlet extensions, JHEP 10 (2012) 195 [arXiv: 1208.1683] [INSPIRE].

[71] F. Boudjema and G.D. La Rochelle, Supersymmetric Higgses beyond the MSSM: An update with flavour and Dark Matter constraints, Phys. Rev. D 86 (2012) 115007 [arXiv: 1208.1952] [INSPIRE].

[72] L. Maiani, A. Polosa and V. Riquer, Heavier Higgs Particles: Indications from Minimal Supersymmetry, Phys. Lett. B 718 (2012) 465 [arXiv:1209.4816] [InSPIRE].

[73] H. Baer et al., Post-LHC7 fine-tuning in the mSUGRA/CMSSM model with a 125 GeV Higgs boson, arXiv:1210.3019 [INSPIRE].

[74] M. Drees, A Supersymmetric Explanation of the Excess of Higgs-like Events at the LHC and at LEP, Phys. Rev. D 86 (2012) 115018 [arXiv:1210.6507] [InSPIRE].

[75] U. Haisch and F. Mahmoudi, MSSM: Cornered and Correlated, JHEP 01 (2013) 061 [arXiv: 1210.7806] [INSPIRE]. 
[76] Tevatron New Physics Higgs Working Group, CDF and D0 collaborations, Updated Combination of CDF and DO Searches for Standard Model Higgs Boson Production with up to $10.0 \mathrm{fb}^{-1}$ of Data, arXiv:1207.0449 [INSPIRE].

[77] CMS collaboration, Higgs to tau tau (MSSM) (HCP), CMS-PAS-HIG-12-050 (2012).

[78] CMS collaboration, MSSM Higgs production in association with b quarks - all hadronic, CMS-PAS-HIG-12-026 (2012).

[79] CMS collaboration, MSSM Higgs production in association with b-quarks - semi leptonic, CMS-PAS-HIG-12-027 (2012).

[80] LHCB collaboration, First evidence for the decay $B_{s} \rightarrow \mu^{+} \mu^{-}$, Phys. Rev. Lett. 110 (2013) 021801 [arXiv: 1211.2674] [INSPIRE].

[81] BeLLE collaboration, Measurement of $B^{-} \rightarrow \tau^{-} \bar{\nu}_{\tau}$ with a Hadronic Tagging Method Using the Full Data Sample of Belle, arXiv:1208.4678 [INSPIRE].

[82] BABAR collaboration, Evidence of $B \rightarrow \tau \nu$ decays with hadronic $B$ tags, arXiv:1207.0698 [INSPIRE].

[83] BABAR collaboration, Measurement of $B\left(B \rightarrow X_{s} \gamma\right)$, the $B \rightarrow X_{s} \gamma$ photon energy spectrum and the direct $C P$ asymmetry in $B \rightarrow X_{s+d} \gamma$ decays, Phys. Rev. D 86 (2012) 112008 [arXiv:1207.5772] [INSPIRE].

[84] XENON100 collaboration, Dark Matter Results from 225 Live Days of XENON100 Data, Phys. Rev. Lett. 109 (2012) 181301 [arXiv:1207.5988] [INSPIRE].

[85] G. D'Ambrosio, G. Giudice, G. Isidori and A. Strumia, Minimal flavor violation: An Effective field theory approach, Nucl. Phys. B 645 (2002) 155 [hep-ph/0207036] [InSPIRE].

[86] R.S. Chivukula and H. Georgi, Composite Technicolor Standard Model, Phys. Lett. B 188 (1987) 99 [INSPIRE].

[87] L. Hall and L. Randall, Weak scale effective supersymmetry, Phys. Rev. Lett. 65 (1990) 2939 [INSPIRE].

[88] A. Buras, P. Gambino, M. Gorbahn, S. Jager and L. Silvestrini, Universal unitarity triangle and physics beyond the standard model, Phys. Lett. B 500 (2001) 161 [hep-ph/0007085] [INSPIRE].

[89] M.S. Carena, A. Menon, R. Noriega-Papaqui, A. Szynkman and C. Wagner, Constraints on $B$ and Higgs physics in minimal low energy supersymmetric models, Phys. Rev. D 74 (2006) 015009 [hep-ph/0603106] [inSPIRE].

[90] M.S. Carena, A. Menon and C. Wagner, Challenges for MSSM Higgs searches at hadron colliders, Phys. Rev. D 76 (2007) 035004 [arXiv:0704.1143] [InSPIRE].

[91] M. Carena, A. Menon and C. Wagner, Minimal Flavor Violation and the Scale of Supersymmetry Breaking, Phys. Rev. D 79 (2009) 075025 [arXiv:0812.3594] [InSPIRE].

[92] P. Paradisi, M. Ratz, R. Schieren and C. Simonetto, Running minimal flavor violation, Phys. Lett. B 668 (2008) 202 [arXiv: 0805. 3989] [INSPIRE].

[93] G. Colangelo, E. Nikolidakis and C. Smith, Supersymmetric models with minimal flavour violation and their running, Eur. Phys. J. C 59 (2009) 75 [arXiv:0807.0801] [inSPIRE].

[94] C.F. Berger, J.S. Gainer, J.L. Hewett and T.G. Rizzo, Supersymmetry Without Prejudice, JHEP 02 (2009) 023 [arXiv: 0812.0980] [inSPIRE]. 
[95] A. Crivellin, Effective Higgs Vertices in the generic MSSM, Phys. Rev. D 83 (2011) 056001 [arXiv: 1012.4840] [INSPIRE].

[96] A. Crivellin, L. Hofer and J. Rosiek, Complete resummation of chirally-enhanced loop-effects in the MSSM with non-minimal sources of flavor-violation, JHEP 07 (2011) 017 [arXiv:1103.4272] [INSPIRE].

[97] M.S. Carena, M. Quirós and C. Wagner, Effective potential methods and the Higgs mass spectrum in the MSSM, Nucl. Phys. B 461 (1996) 407 [hep-ph/9508343] [INSPIRE].

[98] R. Hempfling, Yukawa coupling unification with supersymmetric threshold corrections, Phys. Rev. D 49 (1994) 6168 [inSPIRE].

[99] L.J. Hall, R. Rattazzi and U. Sarid, The Top quark mass in supersymmetric $\mathrm{SO}(10)$ unification, Phys. Rev. D 50 (1994) 7048 [hep-ph/9306309] [INSPIRE].

[100] M.S. Carena, M. Olechowski, S. Pokorski and C. Wagner, Electroweak symmetry breaking and bottom-top Yukawa unification, Nucl. Phys. B 426 (1994) 269 [hep-ph/9402253] [INSPIRE].

[101] B.A. Dobrescu and P.J. Fox, Uplifted supersymmetric Higgs region, Eur. Phys. J. C 70 (2010) 263 [arXiv: 1001.3147] [INSPIRE].

[102] T. Blazek, S. Raby and S. Pokorski, Finite supersymmetric threshold corrections to CKM matrix elements in the large tan Beta regime, Phys. Rev. D 52 (1995) 4151 [hep-ph/9504364] [INSPIRE].

[103] M.S. Carena, D. Garcia, U. Nierste and C.E. Wagner, Effective Lagrangian for the $\bar{t} b H^{+}$ interaction in the MSSM and charged Higgs phenomenology, Nucl. Phys. B 577 (2000) 88 [hep-ph/9912516] [INSPIRE].

[104] M.S. Carena, D. Garcia, U. Nierste and C.E. Wagner, $b \rightarrow s \gamma$ and supersymmetry with large $\tan \beta$, Phys. Lett. B 499 (2001) 141 [hep-ph/0010003] [INSPIRE].

[105] C. Hamzaoui, M. Pospelov and M. Toharia, Higgs mediated FCNC in supersymmetric models with large tan Beta, Phys. Rev. D 59 (1999) 095005 [hep-ph/9807350] [INSPIRE].

[106] K. Babu and C.F. Kolda, Higgs mediated $B^{0} \rightarrow \mu^{+} \mu^{-}$in minimal supersymmetry, Phys. Rev. Lett. 84 (2000) 228 [hep-ph/9909476] [INSPIRE].

[107] G. Isidori and A. Retico, Scalar flavor changing neutral currents in the large $\tan \beta$ limit, JHEP 11 (2001) 001 [hep-ph/0110121] [INSPIRE].

[108] A. Dedes and A. Pilaftsis, Resummed effective Lagrangian for Higgs mediated FCNC interactions in the CP-violating MSSM, Phys. Rev. D 67 (2003) 015012 [hep-ph/0209306] [INSPIRE].

[109] A.J. Buras, P.H. Chankowski, J. Rosiek and L. Slawianowska, $\Delta M_{d, s}, B^{0} d, s \rightarrow \mu^{+} \mu^{-}$and $B \rightarrow X_{s} \gamma$ in supersymmetry at large $\tan \beta$, Nucl. Phys. B 659 (2003) 3 [hep-ph/0210145] [INSPIRE].

[110] L. Hofer, U. Nierste and D. Scherer, Resummation of $\tan \beta$-enhanced supersymmetric loop corrections beyond the decoupling limit, JHEP 10 (2009) 081 [arXiv:0907.5408] [INSPIRE].

[111] CDF collaboration, Search for Higgs bosons predicted in two-Higgs-doublet models via decays to tau lepton pairs in $1.96 \mathrm{TeV}$ p $\bar{p}$ collisions, Phys. Rev. Lett. 103 (2009) 201801 [arXiv: 0906.1014] [INSPIRE]. 
[112] D0 collaboration, Search for Higgs bosons decaying to $\tau \tau$ pairs in p $\bar{p}$ collisions at $\sqrt{s}=1.96 \mathrm{TeV}$, Phys. Lett. B 707 (2012) 323 [arXiv:1106.4555] [INSPIRE].

[113] D0 collaboration, Search for neutral Minimal Supersymmetric Standard Model Higgs bosons decaying to tau pairs produced in association with $b$ quarks in p p collisions at $\sqrt{s}=1.96 \mathrm{TeV}$, Phys. Rev. Lett. 107 (2011) 121801 [arXiv:1106.4885] [INSPIRE].

[114] CDF and D0 collaborations, Search for Neutral Higgs Bosons in Events with Multiple Bottom Quarks at the Tevatron, arXiv:1207.2757 [INSPIRE].

[115] CMS collaboration, Search for neutral Higgs bosons decaying to $\tau$ pairs in pp collisions at $\sqrt{s}=7$ TeV, Phys. Lett. B 713 (2012) 68 [arXiv:1202.4083] [INSPIRE].

[116] ATLAS collaboration, Search for Neutral MSSM Higgs bosons in sqrts $=7 \mathrm{TeV}$ pp collisions at ATLAS, ATLAS-CONF-2012-094 (2012).

[117] D0 collaboration, Search for charged Higgs bosons in top quark decays, Phys. Lett. B 682 (2009) 278 [arXiv: 0908.1811] [INSPIRE].

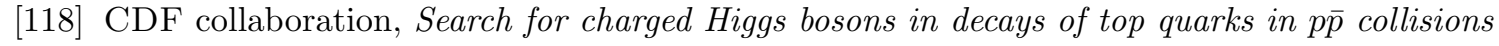
at $\sqrt{s}=1.96 \mathrm{TeV}$, Phys. Rev. Lett. 103 (2009) 101803 [arXiv:0907.1269] [INSPIRE].

[119] ATLAS collaboration, Search for charged Higgs bosons decaying via $H^{+} \rightarrow \tau \nu$ in top quark pair events using pp collision data at $\sqrt{s}=7 \mathrm{TeV}$ with the ATLAS detector, JHEP 06 (2012) 039 [arXiv: 1204.2760] [INSPIRE].

[120] ATLAS collaboration, A Search for a light charged Higgs boson decaying to cs in pp collisions at $\sqrt{s}=7 \mathrm{TeV}$ with the ATLAS detector, ATLAS-CONF-2011-094 (2011).

[121] CMS collaboration, Search for a light charged Higgs boson in top quark decays in pp collisions at $\sqrt{s}=7 \mathrm{TeV}$, JHEP 07 (2012) 143 [arXiv:1205.5736] [INSPIRE].

[122] M. Spira, HIGLU: A program for the calculation of the total Higgs production cross-section at hadron colliders via gluon fusion including QCD corrections, hep-ph/9510347 [INSPIRE].

[123] R.V. Harlander and W.B. Kilgore, Higgs boson production in bottom quark fusion at next-to-next-to leading order, Phys. Rev. D 68 (2003) 013001 [hep-ph/0304035] [INSPIRE].

[124] A. Djouadi, J. Kalinowski and M. Spira, HDECAY: A Program for Higgs boson decays in the standard model and its supersymmetric extension, Comput. Phys. Commun. 108 (1998) 56 [hep-ph/9704448] [inSPIRE].

[125] S. Heinemeyer, W. Hollik and G. Weiglein, FeynHiggs: A Program for the calculation of the masses of the neutral CP even Higgs bosons in the MSSM, Comput. Phys. Commun. 124 (2000) 76 [hep-ph/9812320] [INSPIRE].

[126] M.S. Carena, S. Heinemeyer, C. Wagner and G. Weiglein, Suggestions for benchmark scenarios for MSSM Higgs boson searches at hadron colliders, Eur. Phys. J. C 26 (2003) 601 [hep-ph/0202167] [INSPIRE].

[127] M.S. Carena, S. Heinemeyer, C. Wagner and G. Weiglein, MSSM Higgs boson searches at the Tevatron and the LHC: Impact of different benchmark scenarios, Eur. Phys. J. C 45 (2006) 797 [hep-ph/0511023] [INSPIRE].

[128] M. Carena et al., LHC Discovery Potential for Non-Standard Higgs Bosons in the $3 b$ Channel, JHEP 07 (2012) 091 [arXiv: 1203.1041] [INSPIRE].

[129] J. Hisano and S. Sugiyama, Charge-breaking constraints on left-right mixing of stau's, Phys. Lett. B 696 (2011) 92 [arXiv: 1011.0260] [INSPIRE]. 
[130] M. Claudson, L.J. Hall and I. Hinchliffe, Low-Energy Supergravity: False Vacua and Vacuous Predictions, Nucl. Phys. B 228 (1983) 501 [InSPIRE].

[131] A. Kusenko, P. Langacker and G. Segre, Phase transitions and vacuum tunneling into charge and color breaking minima in the MSSM, Phys. Rev. D 54 (1996) 5824 [hep-ph/9602414] [INSPIRE].

[132] M.J. Duncan and L.G. Jensen, Exact tunneling solutions in scalar field theory, Phys. Lett. B 291 (1992) 109 [INSPIRE].

[133] C.L. Wainwright, CosmoTransitions: Computing Cosmological Phase Transition Temperatures and Bubble Profiles with Multiple Fields, Comput. Phys. Commun. 183 (2012) 2006 [arXiv: 1109.4189] [inSPIRE].

[134] S. Bertolini, F. Borzumati, A. Masiero and G. Ridolfi, Effects of supergravity induced electroweak breaking on rare B decays and mixings, Nucl. Phys. B 353 (1991) 591 [INSPIRE].

[135] T. Goto, Y. Okada and Y. Shimizu, Flavor changing neutral current processes in $B$ and $K$ decays in the supergravity model, Phys. Rev. D 58 (1998) 094006 [hep-ph/9804294] [INSPIRE].

[136] G. Isidori and P. Paradisi, Hints of large $\tan \beta$ in flavour physics, Phys. Lett. B 639 (2006) 499 [hep-ph/0605012] [inSPIRE].

[137] W. Altmannshofer, A.J. Buras and D. Guadagnoli, The MFV limit of the MSSM for low $\tan \beta$ : Meson mixings revisited, JHEP 11 (2007) 065 [hep-ph/0703200] [INSPIRE].

[138] F. Domingo and U. Ellwanger, Updated Constraints from B Physics on the MSSM and the NMSSM, JHEP 12 (2007) 090 [arXiv:0710.3714] [INSPIRE].

[139] M. Wick and W. Altmannshofer, A Reconsideration of the $b \rightarrow s \gamma$ Decay in the Minimal Flavor Violating MSSM, AIP Conf. Proc. 1078 (2009) 348 [arXiv:0810.2874] [INSPIRE].

[140] W. Altmannshofer and D.M. Straub, Viability of MSSM scenarios at very large $\tan \beta$, JHEP 09 (2010) 078 [arXiv: 1004.1993] [INSPIRE].

[141] F. Gabbiani, E. Gabrielli, A. Masiero and L. Silvestrini, A Complete analysis of FCNC and CP constraints in general SUSY extensions of the standard model, Nucl. Phys. B 477 (1996) 321 [hep-ph/9604387] [INSPIRE].

[142] S. Baek, T. Goto, Y. Okada and K.-i. Okumura, Muon anomalous magnetic moment, lepton flavor violation and flavor changing neutral current processes in SUSY GUT with right-handed neutrino, Phys. Rev. D 64 (2001) 095001 [hep-ph/0104146] [INSPIRE].

[143] J. Foster, K.-i. Okumura and L. Roszkowski, Probing the flavor structure of supersymmetry breaking with rare B-processes: A Beyond leading order analysis, JHEP 08 (2005) 094 [hep-ph/0506146] [INSPIRE].

[144] G.F. Giudice, M. Nardecchia and A. Romanino, Hierarchical Soft Terms and Flavor Physics, Nucl. Phys. B 813 (2009) 156 [arXiv:0812.3610] [InSPIRE].

[145] W. Altmannshofer, A.J. Buras, S. Gori, P. Paradisi and D.M. Straub, Anatomy and Phenomenology of FCNC and CPV Effects in SUSY Theories, Nucl. Phys. B 830 (2010) 17 [arXiv: 0909.1333] [INSPIRE].

[146] A. Crivellin and M. Davidkov, Do squarks have to be degenerate? Constraining the mass splitting with Kaon and D mixing, Phys. Rev. D 81 (2010) 095004 [arXiv:1002.2653] [INSPIRE]. 
[147] R. Barbieri, G. Isidori, J. Jones-Perez, P. Lodone and D.M. Straub, U(2) and Minimal Flavour Violation in Supersymmetry, Eur. Phys. J. C 71 (2011) 1725 [arXiv:1105.2296] [INSPIRE].

[148] A. Crivellin, L. Hofer, U. Nierste and D. Scherer, Phenomenological consequences of radiative flavor violation in the MSSM, Phys. Rev. D 84 (2011) 035030 [arXiv:1105.2818] [InSPIRE].

[149] L. Calibbi, R. Hodgkinson, J. Jones Perez, A. Masiero and O. Vives, Flavour and Collider Interplay for SUSY at LHC7, Eur. Phys. J. C 72 (2012) 1863 [arXiv:1111.0176] [InSPIRE].

[150] G. Elor, L.J. Hall, D. Pinner and J.T. Ruderman, Yukawa Unification and the Superpartner Mass Scale, JHEP 10 (2012) 111 [arXiv:1206.5301] [INSPIRE].

[151] W.-S. Hou, Enhanced charged Higgs boson effects in $B^{-} \rightarrow \tau \bar{\nu}, \mu \bar{\nu}$ and $b \rightarrow \tau \bar{\nu}+X$, Phys. Rev. D 48 (1993) 2342 [INSPIRE].

[152] Particle Data Group collaboration, Review of particle physics, J. Phys. G 37 (2010) 075021 [INSPIRE].

[153] C. McNeile, C. Davies, E. Follana, K. Hornbostel and G. Lepage, High-Precision $f_{B_{s}}$ and HQET from Relativistic Lattice QCD, Phys. Rev. D 85 (2012) 031503 [arXiv:1110.4510] [INSPIRE].

[154] Fermilab Lattice and MILC collaborations, B- and D-meson decay constants from three-flavor lattice QCD, Phys. Rev. D 85 (2012) 114506 [arXiv:1112.3051] [INSPIRE].

[155] H. Na et al., The B and $B_{s}$ Meson Decay Constants from Lattice QCD, Phys. Rev. D 86 (2012) 034506 [arXiv: 1202.4914] [INSPIRE].

[156] C. Davies, Standard Model Heavy Flavor physics on the Lattice, PoS (LATTICE 2011) 019 [arXiv: 1203.3862] [INSPIRE].

[157] BABAR collaboration, A Search for $B^{+} \rightarrow \ell^{+} \nu_{\ell}$ Recoiling Against $B^{-} \rightarrow D^{0} \ell^{-} \bar{\nu} X$, arXiv:0809.4027 [INSPIRE].

[158] BeLLe collaboration, Evidence for $B^{-} \rightarrow \tau^{-} \bar{\nu}$ with a Semileptonic Tagging Method, Phys. Rev. D 82 (2010) 071101 [arXiv:1006.4201] [INSPIRE].

[159] M. Tanaka, Charged Higgs effects on exclusive semitauonic B decays, Z. Phys. C 67 (1995) 321 [hep-ph/9411405] [INSPIRE].

[160] U. Nierste, S. Trine and S. Westhoff, Charged-Higgs effects in a new $B \rightarrow D \tau \nu$ differential decay distribution, Phys. Rev. D 78 (2008) 015006 [arXiv:0801.4938] [inSPIRE].

[161] J.F. Kamenik and F. Mescia, $B \rightarrow D \tau \nu$ Branching Ratios: Opportunity for Lattice QCD and Hadron Colliders, Phys. Rev. D 78 (2008) 014003 [arXiv:0802.3790] [INSPIRE].

[162] S. Fajfer, J.F. Kamenik and I. Nisandzic, On the $B \rightarrow D^{*} \tau \bar{\nu}_{\tau}$ Sensitivity to New Physics, Phys. Rev. D 85 (2012) 094025 [arXiv:1203.2654] [INSPIRE].

[163] D. Becirevic, N. Kosnik and A. Tayduganov, $\bar{B} \rightarrow D \tau \bar{\nu}_{\tau}$ vs. $\bar{B} \rightarrow D \mu \bar{\nu}_{\mu}$, Phys. Lett. B 716 (2012) 208 [arXiv:1206. 4977] [INSPIRE].

[164] J.A. Bailey et al., Refining new-physics searches in $B \rightarrow D \tau \nu$ decay with lattice $Q C D$, Phys. Rev. Lett. 109 (2012) 071802 [arXiv:1206.4992] [INSPIRE].

[165] BaBAr collaboration, Evidence for an excess of $\bar{B} \rightarrow D^{(*)} \tau^{-} \bar{\nu}_{\tau}$ decays, Phys. Rev. Lett. 109 (2012) 101802 [arXiv:1205.5442] [INSPIRE]. 
[166] BeLLe collaboration, Observation of $B^{+} \rightarrow \bar{D}^{* 0} \tau^{+} \nu_{\tau}$ and Evidence for $B^{+} \rightarrow \bar{D}^{0} \tau^{+} \nu_{\tau}$ at Belle, Phys. Rev. D 82 (2010) 072005 [arXiv: 1005. 2302] [INSPIRE].

[167] M. Antonelli et al., An Evaluation of $\left|\mathrm{V}_{\mathrm{us}}\right|$ and precise tests of the Standard Model from world data on leptonic and semileptonic kaon decays, Eur. Phys. J. C 69 (2010) 399 [arXiv: 1005.2323] [INSPIRE].

[168] S. Fajfer, J.F. Kamenik, I. Nisandzic and J. Zupan, Implications of Lepton Flavor Universality Violations in B Decays, Phys. Rev. Lett. 109 (2012) 161801 [arXiv:1206.1872] [INSPIRE].

[169] A. Crivellin, C. Greub and A. Kokulu, Explaining $B \rightarrow D \tau \nu, B \rightarrow D^{*} \tau \nu$ and $B \rightarrow \tau \nu$ in a 2HDM of type-III, Phys. Rev. D 86 (2012) 054014 [arXiv:1206.2634] [InSPIRE].

[170] A. Datta, M. Duraisamy and D. Ghosh, Diagnosing New Physics in $b \rightarrow c \tau \nu_{\tau}$ decays in the light of the recent BaBar result, Phys. Rev. D 86 (2012) 034027 [arXiv:1206.3760] [INSPIRE].

[171] N. Deshpande and A. Menon, Hints of R-parity violation in B decays into $\tau \nu$, arXiv: 1208.4134 [INSPIRE].

[172] A. Celis, M. Jung, X.-Q. Li and A. Pich, Sensitivity to charged scalars in $B \rightarrow D^{(*)} \tau \nu_{\tau}$ and $B \rightarrow \tau \nu_{\tau}$ decays, JHEP 01 (2013) 054 [arXiv: 1210.8443] [INSPIRE].

[173] K. De Bruyn et al., Branching Ratio Measurements of $B_{s}$ Decays, Phys. Rev. D 86 (2012) 014027 [arXiv: 1204.1735] [inSPIRE].

[174] K. De Bruyn et al., Probing New Physics via the $B_{s}^{0} \rightarrow \mu^{+} \mu^{-}$Effective Lifetime, Phys. Rev. Lett. 109 (2012) 041801 [arXiv: 1204.1737] [inSPIRE].

[175] W. Altmannshofer and D.M. Straub, Cornering New Physics in $b \rightarrow s$ Transitions, JHEP 08 (2012) 121 [arXiv: 1206. 0273] [INSPIRE].

[176] A.J. Buras, J. Girrbach, D. Guadagnoli and G. Isidori, On the Standard Model prediction for BR(Bs,d to $\left.\mu^{+} \mu^{-}\right)$, Eur. Phys. J. C 72 (2012) 2172 [arXiv:1208.0934] [INSPIRE].

[177] D0 collaboration, Search for the rare decay $B_{s}^{0} \rightarrow m u^{+} m u^{-}$, Phys. Lett. B 693 (2010) 539 [arXiv: 1006.3469] [INSPIRE].

[178] CDF collaboration, Search for $B_{s} \rightarrow \mu^{+} \mu^{-}$and $B_{d} \rightarrow \mu^{+} \mu^{-}$Decays with CDF II, Phys. Rev. Lett. 107 (2011) 239903 [arXiv:1107.2304] [INSPIRE].

[179] ATLAS collaboration, Search for the decay $B_{s}^{0} \rightarrow \mu \mu$ with the ATLAS detector, Phys. Lett. B 713 (2012) 387 [arXiv: 1204.0735] [inSPIRE].

[180] CMS collaboration, Search for $B_{s}^{0}$ to $\mu^{+} \mu^{-}$and $B^{0}$ to $\mu^{+} \mu^{-}$decays, JHEP 04 (2012) 033 [arXiv:1203.3976] [INSPIRE].

[181] LHCB collaboration, Strong constraints on the rare decays $B_{s} \rightarrow \mu^{+} \mu^{-}$and $B^{0} \rightarrow \mu^{+} \mu^{-}$, Phys. Rev. Lett. 108 (2012) 231801 [arXiv:1203.4493] [INSPIRE].

[182] LHCB, CMS and ATLAS collaborations, Search for the rare decays $B_{(s)}^{0} \rightarrow \mu^{+} \mu^{-}$at the LHC with the ATLAS, CMS and LHCb experiments, LHCb-CONF-2012-017 (2012).

[183] S.R. Choudhury and N. Gaur, Dileptonic decay of B(s) meson in SUSY models with large tan Beta, Phys. Lett. B 451 (1999) 86 [hep-ph/9810307] [INSPIRE].

[184] DELPHI collaboration, Searches for supersymmetric particles in $e^{+} e^{-}$collisions up to 208-GeV and interpretation of the results within the MSSM, Eur. Phys. J. C 31 (2003) 421 [hep-ex/0311019] [INSPIRE]. 
[185] OPAL collaboration, Search for chargino and neutralino production at $\sqrt{s}=192$ GeV to 209 GeV at LEP, Eur. Phys. J. C 35 (2004) 1 [hep-ex/0401026] [INSPIRE].

[186] LHCB collaboration, Framework TDR for the LHCb Upgrade: Technical Design Report, CERN-LHCC-2012-007 (2012).

[187] H.E. Logan and U. Nierste, $B_{s, d} \rightarrow \ell^{+} \ell^{-}$in a two Higgs doublet model, Nucl. Phys. B 586 (2000) 39 [hep-ph/0004139] [INSPIRE].

[188] M. Misiak et al., Estimate of $B\left(\bar{B} \rightarrow X_{s} \gamma\right)$ at $O\left(\alpha_{s}^{2}\right)$, Phys. Rev. Lett. 98 (2007) 022002 [hep-ph/0609232] [INSPIRE].

[189] T. Becher and M. Neubert, Analysis of $\operatorname{Br}\left(\bar{B} \rightarrow X_{s} \gamma\right)$ at NNLO with a cut on photon energy, Phys. Rev. Lett. 98 (2007) 022003 [hep-ph/0610067] [InSPIRE].

[190] M. Benzke, S.J. Lee, M. Neubert and G. Paz, Factorization at Subleading Power and Irreducible Uncertainties in $\bar{B} \rightarrow X_{s} \gamma$ Decay, JHEP 08 (2010) 099 [arXiv:1003.5012] [INSPIRE].

[191] Heavy Flavor Averaging Group collaboration, Y. Amhis et al., Averages of b-hadron, c-hadron and tau-lepton properties as of early 2012, arXiv:1207.1158 [INSPIRE].

[192] A. Freitas and U. Haisch, $\bar{B} \rightarrow X_{s} \gamma$ in two universal extra dimensions, Phys. Rev. D 77 (2008) 093008 [arXiv: 0801.4346 ] [INSPIRE].

[193] W. Altmannshofer, P. Paradisi and D.M. Straub, Model-Independent Constraints on New Physics in $b \rightarrow s$ Transitions, JHEP 04 (2012) 008 [arXiv:1111.1257] [INSPIRE].

[194] S.P. Martin and M.T. Vaughn, Two loop renormalization group equations for soft supersymmetry breaking couplings, Phys. Rev. D 50 (1994) 2282 [Erratum ibid. D 78 (2008) 039903] [hep-ph/9311340] [INSPIRE].

[195] D.M. Pierce, J.A. Bagger, K.T. Matchev and R.-j. Zhang, Precision corrections in the minimal supersymmetric standard model, Nucl. Phys. B 491 (1997) 3 [hep-ph/9606211] [INSPIRE].

[196] L.E. Ibáñez and C. Lopez, $N=1$ Supergravity, the Weak Scale and the Low-Energy Particle Spectrum, Nucl. Phys. B 233 (1984) 511 [inSPIRE].

[197] L.E. Ibáñez, C. Lopez and C. Muñoz, The Low-Energy Supersymmetric Spectrum According to $N=1$ Supergravity Guts, Nucl. Phys. B 256 (1985) 218 [inSPIRE].

[198] M.S. Carena, M. Olechowski, S. Pokorski and C. Wagner, Radiative electroweak symmetry breaking and the infrared fixed point of the top quark mass, Nucl. Phys. B 419 (1994) 213 [hep-ph/9311222] [INSPIRE].

[199] N. Arkani-Hamed, A. Delgado and G. Giudice, The Well-tempered neutralino, Nucl. Phys. B 741 (2006) 108 [hep-ph/0601041] [INSPIRE].

[200] G.B. Gelmini and P. Gondolo, Neutralino with the right cold dark matter abundance in (almost) any supersymmetric model, Phys. Rev. D 74 (2006) 023510 [hep-ph/0602230] [INSPIRE].

[201] M. Srednicki and R. Watkins, Coherent couplings of neutralinos to nuclei from squark mixing, Phys. Lett. B 225 (1989) 140 [InSPIRE].

[202] G. Gelmini, P. Gondolo and E. Roulet, Neutralino dark matter searches, Nucl. Phys. B 351 (1991) 623 [INSPIRE]. 
[203] M. Drees and M. Nojiri, Neutralino-nucleon scattering revisited, Phys. Rev. D 48 (1993) 3483 [hep-ph/9307208] [INSPIRE].

[204] J.R. Ellis, A. Ferstl and K.A. Olive, Reevaluation of the elastic scattering of supersymmetric dark matter, Phys. Lett. B 481 (2000) 304 [hep-ph/0001005] [INSPIRE].

[205] J.R. Ellis, K.A. Olive, Y. Santoso and V.C. Spanos, Update on the direct detection of supersymmetric dark matter, Phys. Rev. D 71 (2005) 095007 [hep-ph/0502001] [INSPIRE].

[206] M.S. Carena, D. Hooper and P.Z. Skands, Implications of direct dark matter searches for MSSM Higgs searches at the Tevatron, Phys. Rev. Lett. 97 (2006) 051801 [hep-ph/0603180] [INSPIRE].

[207] M.S. Carena, D. Hooper and A. Vallinotto, The Interplay Between Collider Searches For Supersymmetric Higgs Bosons and Direct Dark Matter Experiments, Phys. Rev. D 75 (2007) 055010 [hep-ph/0611065] [INSPIRE].

[208] C. Strege et al., Updated global fits of the CMSSM including the latest LHC SUSY and Higgs searches and XENON100 data, JCAP 03 (2012) 030 [arXiv:1112.4192] [INSPIRE].

[209] M. Perelstein and B. Shakya, XENON100 Implications for Naturalness in the MSSM, NMSSM and lambda-SUSY, arXiv:1208.0833 [INSPIRE].

[210] J. Giedt, A.W. Thomas and R.D. Young, Dark matter, the CMSSM and lattice QCD, Phys. Rev. Lett. 103 (2009) 201802 [arXiv:0907.4177] [INSPIRE].

[211] XENON100 collaboration, Dark Matter Results from 100 Live Days of XENON100 Data, Phys. Rev. Lett. 107 (2011) 131302 [arXiv:1104.2549] [INSPIRE]. 